\title{
A search for flavour changing neutral currents in top-quark decays in $p p$ collision data collected with the ATLAS detector at $\sqrt{s}=7 \mathrm{TeV}$
}

\section{The ATLAS collaboration}

\author{
CERN, Geneva, Switzerland \\ E-mail: atlas.publications@cern.ch
}

ABSTRACT: A search for flavour changing neutral current (FCNC) processes in top-quark decays by the ATLAS Collaboration is presented. Data collected from $p p$ collisions at the LHC at a centre-of-mass energy of $\sqrt{s}=7 \mathrm{TeV}$ during 2011, corresponding to an integrated luminosity of $2.1 \mathrm{fb}^{-1}$, were used. A search was performed for top-quark pair-production events, with one top quark decaying through the $t \rightarrow Z q$ FCNC $(q=u, c)$ channel, and the other through the Standard Model dominant mode $t \rightarrow W b$. Only the decays of the $Z$ boson to charged leptons and leptonic $W$-boson decays were considered as signal. Consequently, the final-state topology is characterised by the presence of three isolated charged leptons, at least two jets and missing transverse momentum from the undetected neutrino. No evidence for an FCNC signal was found. An upper limit on the $t \rightarrow Z q$ branching ratio of $\mathrm{BR}(t \rightarrow Z q)<0.73 \%$ is set at the $95 \%$ confidence level.

Keywords: Hadron Hadron Scattering, Top Physics, Rare Decays, Flavour Changing Neutral Currents 


\section{Contents}

1 Introduction $\quad 1$

2 Detector and data samples $\quad 2$

3 Monte Carlo simulation samples $\quad 3$

3.1 Signal 3

3.2 Background 3

4 Object definition $\quad 4$

5 Event selection and reconstruction $\quad 6$

$\begin{array}{llr}6 & \text { Background evaluation } & 8\end{array}$

$\begin{array}{lll}7 & \text { Systematic uncertainties } & 10\end{array}$

8 Limit evaluation $\quad 13$

9 Conclusions $\quad 14$

$\begin{array}{ll}\text { The ATLAS collaboration } & 20\end{array}$

\section{Introduction}

The top quark is the heaviest known elementary particle, with a mass of $m_{t}=173.2 \pm$ $0.9 \mathrm{GeV}$ [1]. The very large mass may provide a window onto physics beyond the Standard Model (SM). Deviations from SM predictions of the production and decay properties of the top quark provide model-independent tests for physics beyond the SM. According to the SM, the top quark decays nearly $100 \%$ of the time to a $W$ boson and a $b$ quark. Flavour changing neutral current (FCNC) decays are highly suppressed in the SM by the GIM mechanism [2] with a branching ratio (BR) of the order of $10^{-14}$.

Several SM extensions predict a higher BR for top-quark FCNC decays. Examples of such extensions are the quark-singlet model [3-5], the two-Higgs doublet model with or without flavour-conservation [6-11], the minimal supersymmetric model [12-18], supersymmetry (SUSY) with $R$-parity violation [19], the topcolour-assisted technicolour model [20] or models with warped extra dimensions [21, 22]. The top-quark FCNC decay BR in these models is typically many orders of magnitude larger than the SM BR, and can be as high as $\sim 2 \times 10^{-4}$ in certain $R$-parity violating SUSY models.

Existing experimental limits on top-quark FCNC decays come from direct and indirect searches at the Tevatron collider [23-27], and indirect searches at the LEP [28-33] and 
HERA [34-37] colliders, and at the LHC [38]. The best current direct search limits on the top quark FCNC branching fraction are $3.2 \%$ for both $t \rightarrow q \gamma$ [23] and $t \rightarrow Z q$ $(q=u, c)[27]$.

This article reports a search for FCNC top-quark decays in $t \bar{t}$ events. Events were searched for in which either the top or antitop quark has decayed into a $Z$ boson and a quark, $t \rightarrow Z q$, while the remaining top or antitop quark decayed through the SM $t \rightarrow W b$ channel. Given the current best limit on the $t \rightarrow Z q$ branching fraction of $3.2 \%$, events in which both the top and antitop decay to $Z q$ happen at less than a level of $10^{-3}$, and have no observable effect on this result. Only leptonic decays of the $Z$ and $W$ bosons were considered, yielding a final-state topology characterised by the presence of three isolated charged leptons, at least two jets, and transverse momentum imbalance $\left(E_{\mathrm{T}}^{\mathrm{miss}}\right)$ from the undetected neutrino arising from the $W$-boson decay. Leptons are either well-identified electron or muon candidates, selected using the full detector or, to increase signal acceptance, isolated tracks. Channels with $\tau$ leptons are not explicitly reconstructed, but reconstructed electrons and muons can arise from leptonic $\tau$ decays, and an isolated track can arise from hadronic $\tau$ decay modes.

\section{Detector and data samples}

The ATLAS detector [39] at the LHC covers nearly the entire solid angle around the collision point. It consists of an inner tracking detector comprising a silicon pixel detector, a silicon microstrip detector (SCT), and a transition radiation tracker. The inner detector covers the pseudorapidity ${ }^{1}$ range $|\eta|<2.5$ and is surrounded by a thin superconducting solenoid providing a $2 \mathrm{~T}$ axial magnetic field, and by lead/liquid-argon (LAr) electromagnetic (EM) sampling calorimeters with high granularity. An iron/scintillator-tile calorimeter provides hadronic energy measurements in the central pseudorapidity range $(|\eta|<1.7)$. The end-cap and forward regions are instrumented with LAr calorimeters for both EM and hadronic energy measurements up to $|\eta|<4$.9. The calorimeter system is surrounded by a muon spectrometer incorporating three superconducting toroid magnet assemblies (one barrel and two end-caps), with bending power between $2.0 \mathrm{Tm}$ and 7.5 Tm.

A three-level trigger system is used to collect data. The first-level trigger is implemented in hardware and uses a subset of the detector information to reduce the rate to at most $75 \mathrm{kHz}$. This is followed by two software-based trigger levels that together reduce the event rate to $\sim 300 \mathrm{~Hz}$. This analysis uses inclusive single-muon and single-electron triggers with $p_{\mathrm{T}}$ thresholds of $18 \mathrm{GeV}$ for muons and $20 \mathrm{GeV}$ or $22 \mathrm{GeV}$ for electrons, depending on the data taking period.

Proton-proton collision data taken at $\sqrt{s}=7 \mathrm{TeV}$ by ATLAS between March and August 2011 are used. The data sample corresponds to a total integrated luminosity of $2.1 \mathrm{fb}^{-1}$ with an uncertainty of $3.7 \%[40,41]$. The mean number of interactions per bunch crossing was 6.2 for the full data sample.

\footnotetext{
${ }^{1}$ In the right-handed ATLAS coordinate system, the pseudorapidity $\eta$ is defined as $\eta=-\ln [\tan (\theta / 2)]$, where the polar angle $\theta$ is measured with respect to the LHC beamline. The azimuthal angle $\phi$ is measured with respect to the $x$-axis, which points towards the centre of the LHC ring. The $y$-axis points upwards. Transverse momentum and energy are defined as $p_{\mathrm{T}}=p \sin \theta$ and $E_{\mathrm{T}}=E \sin \theta$, respectively.
} 


\section{Monte Carlo simulation samples}

Monte Carlo (MC) samples were generated to model both FCNC signal events and certain backgrounds. Alternative MC samples were also generated to evaluate various systematic uncertainties. All generated events are propagated through a detailed GEANT4 simulation $[42,43]$ of the ATLAS detector and are reconstructed with the same algorithms as the data. The effect of additional $p p$ interactions in the same bunch crossing as the events of interest was simulated by superimposing additional simulated minimum-bias interactions. The effect from events in neighbouring bunch crossings was also simulated. The simulated events were reweighted such that the average number of extra interactions per crossing (pile-up) matched the data. Data-to-MC scale factors were applied to the MC samples to account for small differences in efficiencies between data and MC simulation.

\subsection{Signal}

Monte Carlo simulation samples of top-quark pair production, with one of the top quarks decaying through FCNC to $Z q$ while the other decays according to the SM, were generated with TopReX [44]. The anomalous coupling in the dimension 5 effective Lagrangian [33] was set to $\kappa_{t q}^{Z}=0.1$ with the energy scale $\Lambda=1 \mathrm{TeV}$. The dimension 4 effective Lagrangian for $t \rightarrow Z q$ was not included. It was checked that the acceptance of top quark FCNC decays was insensitive to the value of $\kappa_{t q}^{Z}$ over the range from .001 to 0.6 , where the lower value corresponds to an FCNC branching fraction of order $10^{-6}$ and the higher value to a branching fraction of nearly $40 \%$, which is already ruled out by previous measurements. It was also checked that inclusion of the dimension 4 Lagrangian does not change the acceptance.

Only decays of the $W$ and $Z$ bosons involving charged leptons were generated $(Z \rightarrow$ $e e, \mu \mu, \tau \tau$ and $W \rightarrow e \nu, \mu \nu, \tau \nu)$. The MRST2007 LO* [45] parton distribution function (PDF) set was used with the TopReX generator. All signal events were hadronised with PYTHIA 6.421 [46]. The masses of the top quark, $W$ boson and $Z$ boson were set to $172.5 \mathrm{GeV}, 80.4 \mathrm{GeV}$ and $91.2 \mathrm{GeV}$, respectively.

To study the effect of the uncertainty due to the top-quark mass, samples with topquark masses of $170 \mathrm{GeV}$ and $175 \mathrm{GeV}$ were also generated. The uncertainty due to initial- and final-state radiation (ISR/FSR) was evaluated using the AcerMC generator [47] interfaced to PYTHIA, and by varying the parameters controlling ISR and FSR in a range consistent with those used in the Perugia Hard/Soft tune variations [48].

\subsection{Background}

Several SM processes have final-state topologies similar to the signal. These include events with three final-state charged leptons (real leptons), as well as events in which at least one jet (including jets with heavy-flavour decays) is misidentified as an isolated charged lepton ('fake leptons') and events with four leptons in which one is not reconstructed.

Diboson events ( $W W, W Z$ and $Z Z$ ) were produced using ALPGEN 2.13 [49]. Up to three additional partons from the matrix element were simulated, and the CTEQ6L1 [50] PDF was used. The parton shower and the underlying event were added using HERWIG v6.510 [51, 52] and the JIMMY [53] underlying event model with the AUET1 tune [54] to 
the ATLAS data. The ALPGEN program with HERWIG showering and the JIMMY underlying event model was also used to generate $Z / \gamma+$ jets.

The $t \bar{t}$ and single-top events were generated using the MC@NLO generator v3.41 [55-57] with the CTEQ6.6 [58] PDFs. The parton shower and the underlying event were added using HERWIG v6.510 and JIMMY generators as described above. The $t \bar{t}$ production cross section was normalized to the approximate next-to-next-to-leading-order (NNLO) prediction of $164.6 \mathrm{pb}$, obtained using the HATHOR tool [59]. The cross sections for single-top production were normalized to the approximate NNLO predictions of $64.6 \mathrm{pb}$ [60], $4.6 \mathrm{pb}$ [61] and $15.7 \mathrm{pb}$ [62] for $t$-channel, $s$-channel and associated $W t$ production, respectively.

Events with $t \bar{t}+W$ and $t \bar{t}+Z$ production, including those with extra jets in the final state, were generated using MADGRAPH 4.4.62 [63]. Parton showering was added using PYTHIA.

All decay modes of the $W$ and $Z$ bosons to charged leptons were considered in the generation and simulation of the background samples used.

Backgrounds that include fake leptons were evaluated using a data-driven approach described below.

\section{Object definition}

The selection of leptons, jets, and $E_{\mathrm{T}}^{\text {miss }}$ was close to that used for the ATLAS measurement of the $t \bar{t}$ production cross section in the dilepton channel [64]. Leptons were selected either using the full ATLAS detector, including the inner detector, calorimeter and muon spectrometer ('identified leptons' or 'ID leptons'), or using only a high quality inner detector track ('track leptons' or 'TLs'). The inclusion of TLs increased the acceptance for $W \rightarrow \tau \nu$ decays, and for electrons and muons that fail the ID lepton selection criteria. TLs were required to be distinct from ID leptons, and at most one TL per event was allowed. Signal candidates selected with three identified leptons are referred to as '3ID' events, and those selected with two identified leptons and a TL are referred to as '2ID+TL' events. The 2ID+TL events increased the signal acceptance by $22 \%$ compared to a 3ID selection alone.

ID electron candidates were reconstructed from energy deposits (clusters) in the EM calorimeter, which were then associated to reconstructed tracks of charged particles in the inner detector. Stringent quality requirements on the conditions of the EM calorimeter at the time of data taking were applied to ensure a well-measured reconstructed energy. A 'tight' selection [65] using calorimeter, tracking and combined variables, was employed to provide good separation between the signal electrons and background. Electron candidates were additionally required to have $\left|\eta_{\mathrm{cl}}\right|<2.47$, excluding electrons in the transition region between the barrel and endcap calorimeters defined by $1.37<\left|\eta_{\mathrm{cl}}\right|<1.52$. The variable $\eta_{\mathrm{cl}}$ is the pseudorapidity of the energy cluster associated with the candidate.

ID muon candidate reconstruction began by searching for track segments in layers of the muon chambers. These segments were combined starting from the outermost layer, fitted to account for material effects, and matched with tracks found in the inner detector. The candidates were refitted using the complete track information from both detector systems, and were required to satisfy $|\eta|<2.5$. 
Candidates for TL were defined by an inner-detector track and a series of quality cuts optimised for high efficiency and a low rate of misidentification. The track was required to have at least six pixel and/or SCT hits and at least one hit in the innermost pixel layer. The transverse distance of closest approach of the track to the beamline, $d_{0}$, was required to satisfy $\left|d_{0}\right|<0.2 \mathrm{~mm}$ and the uncertainty on the momentum measurement was required be less than $20 \%$.

All leptons were required to be isolated and have high transverse momentum, $p_{\mathrm{T}}$, consistent with originating from $W$ - or $Z$-boson decay. Because of the requirement of three leptons in this analysis, lepton thresholds were reduced from those used in ref. [64]. In 3ID events, the leading lepton was required to have $p_{\mathrm{T}}>25 \mathrm{GeV}$, and the two sub-leading leptons were required to have $p_{\mathrm{T}}>20 \mathrm{GeV}$. In 2ID+TL events, the TL was required to have $p_{\mathrm{T}}>25 \mathrm{GeV}$, and the two ID leptons in the event were required to have $p_{\mathrm{T}}>20 \mathrm{GeV}$. At least one ID lepton was required to have fired the trigger. The lepton $p_{\mathrm{T}}$ thresholds were chosen to be consistent with leptons from $W$ and $Z$ boson decays and such that the efficiency does not depend strongly on the lepton $p_{\mathrm{T}}$. To ensure this was the case with the higher electron trigger thresholds (see section 2), reconstructed electrons that were associated with trigger objects were required to have $p_{\mathrm{T}}>25 \mathrm{GeV}$.

Lepton isolation requirements reduce backgrounds from misidentified jets and suppress the selection of leptons from heavy-flavour decays. For ID electron candidates, $E_{\mathrm{T}}$ deposited in the calorimeter cells but not associated to the electron was summed in a cone with radius ${ }^{2} \Delta R=0.2$ around the electron and required to be less than $3.5 \mathrm{GeV}$. For ID muon candidates, the isolation requirement was based on both calorimeter and track information. The track isolation requirement was based on the sum of the track transverse momenta, for tracks with $p_{\mathrm{T}}>1 \mathrm{GeV}$ in a cone with radius $\Delta R=0.3$ centred on the muon candidate, while the calorimeter isolation requirement was based on the transverse energy in the same cone. Both the track and calorimeter sums, excluding the muon candidate, were required to be less than $4 \mathrm{GeV}$. Additionally, ID muon candidates were required to have a distance $\Delta R>0.4$ from any jet with $p_{\mathrm{T}}>20 \mathrm{GeV}$, further suppressing muon candidates from heavy-flavour decays. For TLs, the track was required to be isolated from other nearby tracks following the track isolation definition above, in this case using tracks with $p_{\mathrm{T}}>0.5 \mathrm{GeV}$. The summed momentum cut was set to $2 \mathrm{GeV}$. ID muon candidates arising from cosmic rays were rejected by removing candidate pairs that were back-toback in the $r-\phi$ plane and with transverse impact parameters relative to the beam axis $\left|d_{0}\right|>0.5 \mathrm{~mm}$.

Jets were reconstructed with the anti- $k_{t}$ algorithm [66] with a radius parameter $R=$ 0.4 , starting from energy clusters in the calorimeter reconstructed using the scale established for electromagnetic objects. These jets were then calibrated to the hadronic energy scale using $p_{\mathrm{T}^{-}}$and $\eta$-dependent correction factors [67]. Jets were removed if they were within $\Delta R<0.2$ of a well-identified electron candidate, or within $\Delta R<0.4$ of a TL. The jets used in the analysis were required to have $p_{\mathrm{T}}>25 \mathrm{GeV}$ and $|\eta|<2.5$.

To suppress backgrounds in which TLs are reconstructed from fake leptons, a jet consistent with originating from a $b$ quark was required in events with a TL. Jets were

${ }^{2} \Delta R \equiv \sqrt{(\Delta \eta)^{2}+(\Delta \phi)^{2}}$ 
identified as $b$-quark candidates (' $b$-tagged') by an algorithm that forms a likelihood ratio of $b$ - to light-quark jet hypotheses using several kinematic variables [68]. The cut on the combined likelihood ratio was chosen such that a $b$-tagging efficiency of $\approx 80 \%$ per $b$-jet in $t \bar{t}$ candidate events was achieved.

The $E_{\mathrm{T}}^{\mathrm{miss}}$ vector was formed from the negative vector sum of the transverse momenta of the reconstructed objects (electrons, muons, jets) [69]. The contribution from cells associated with electron candidates was replaced by the calibrated transverse energy of the candidate. The contribution from all ID muon candidates and calorimeter clusters (including those not belonging to a reconstructed object) was also included. TL candidates that arise from muons and leave little energy in the calorimeter are not properly included in the $E_{\mathrm{T}}^{\text {miss }}$. In such events the $E_{\mathrm{T}}^{\text {miss }}$ often points close to the TL direction. In these events the $E_{\mathrm{T}}^{\text {miss }}$ was corrected with the $p_{\mathrm{T}}$ of the TL if the TL and an oppositely-charged ID muon were consistent with coming from a $Z$-boson decay, and if the $\Delta \phi$ between the $E_{\mathrm{T}}^{\text {miss }}$ and the TL direction was less than 0.15 and there is no ID lepton within $\Delta R=0.05$ of the TL (in which case the correction was already included by the $E_{\mathrm{T}}^{\text {miss }}$ algorithm). After all corrections, $E_{\mathrm{T}}^{\text {miss }}>20 \mathrm{GeV}$ was required.

\section{Event selection and reconstruction}

The analysis required collision data selected by an inclusive single-electron or single-muon trigger. To ensure that the event was triggered by the lepton candidates used in the analysis, one of the identified leptons and the triggered lepton were required to match within $\Delta R<0.15$.

Events were required to have a primary interaction vertex with at least five tracks with $p_{\mathrm{T}}>400 \mathrm{MeV}$. The event was discarded if it had any jet with $p_{\mathrm{T}}>20 \mathrm{GeV}$ that failed quality cuts designed to reject jets arising from calorimeter noise or activity inconsistent with the bunch-crossing time [67]. If an electron candidate and a muon candidate shared a track, the event was also discarded.

During part of the data-taking period, corresponding to an integrated luminosity of $0.9 \mathrm{fb}^{-1}$, an electronics failure in a small $\eta-\phi$ region of the LAr EM calorimeter created a dead region. For this subset of the data, events in data and MC simulation containing either an identified electron or a jet with $p_{\mathrm{T}}>20 \mathrm{GeV}$, satisfying $-0.1<\eta<1.5$ and $-0.9<\phi<-0.5$ were rejected.

Events were selected as either 3ID or 2ID+TL candidates, each with thresholds described in section 4 . In both cases, all three lepton candidates were required to come from the same primary interaction vertex. Events were required to have a same-flavour, opposite-sign lepton pair with an invariant mass within $15 \mathrm{GeV}$ of $m_{Z}=91.2 \mathrm{GeV}$. For this purpose a TL can be used with any opposite-sign identified lepton, since its flavour is not known. In addition, signal candidates were required to have at least two jets and $E_{\mathrm{T}}^{\mathrm{miss}}>$ $20 \mathrm{GeV}$. In events selected with a TL, at least one jet was required to be $b$-tagged. Figure 1 shows the $E_{\mathrm{T}}^{\text {miss }}$ distribution for the 3ID and 2ID+TL events prior to the final selection requirements. For the 3ID case these are events with three identified leptons with at least one opposite-sign, same-flavour pair with the $m_{Z}$ invariant mass cut applied, but no jets 


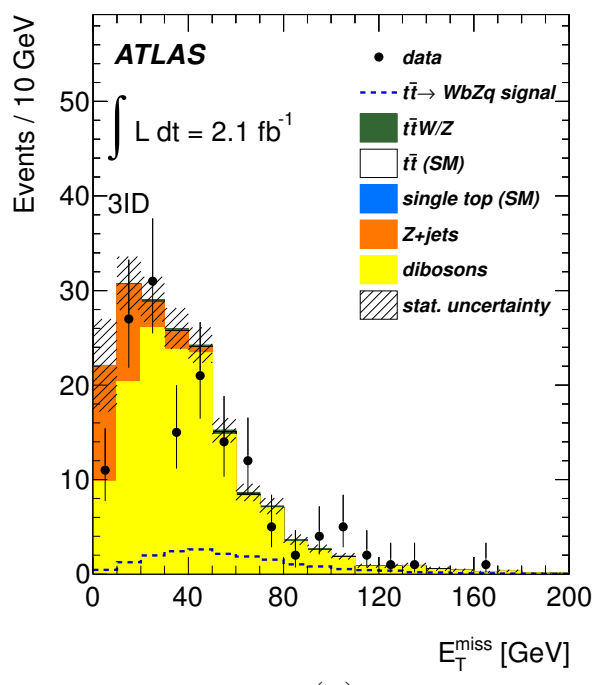

(a)

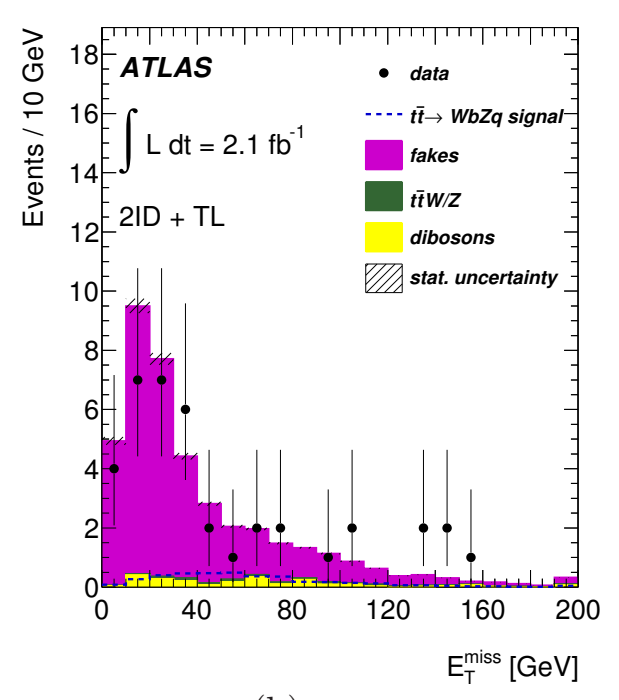

(b)

Figure 1. $E_{\mathrm{T}}^{\mathrm{miss}}$ distributions before the final selection for the (a) 3ID and (b) 2ID+TL analysis. For the 3ID case these are events with three identified leptons with at least one opposite-sign, same-flavour pair with an invariant mass consistent with a $Z$-boson, but no jets or $E_{\mathrm{T}}^{\text {miss }}$ requirement. In the 2ID+TL case, these are events with an opposite-sign pair and both the jet and $b$-tag requirements, but no $Z$-boson or $E_{\mathrm{T}}^{\text {miss }}$ requirements. The uncertainties shown are statistical only. The $t \bar{t} \rightarrow W b Z q$ distributions are normalized to the observed limit in each channel.

or $E_{\mathrm{T}}^{\text {miss }}$ requirement. In the $2 \mathrm{ID}+\mathrm{TL}$ case, these are events with an opposite-sign pair and both the jet and $b$-tag requirements, but no $m_{Z}$ or $E_{\mathrm{T}}^{\text {miss }}$ requirements.

Selected events were required to be kinematically consistent with $t \bar{t} \rightarrow W b Z q$ through a $\chi^{2}$ minimized with respect to jet and lepton assignments and the longitudinal momentum of the neutrino, $p_{z}^{\nu}$. The $\chi^{2}$ was defined as follows

$$
\chi^{2}=\frac{\left(m_{j_{a} \ell_{a} \ell_{b}}^{\mathrm{reco}}-m_{t}\right)^{2}}{\sigma_{t}^{2}}+\frac{\left(m_{j_{b} \ell_{c} \nu}^{\mathrm{reco}}-m_{t}\right)^{2}}{\sigma_{t}^{2}}+\frac{\left(m_{\ell_{c} \nu}^{\mathrm{reco}}-m_{W}\right)^{2}}{\sigma_{W}^{2}}+\frac{\left(m_{\ell_{a} \ell_{b}}^{\mathrm{reco}}-m_{Z}\right)^{2}}{\sigma_{Z}^{2}},
$$

where $j_{a, b}$ are the two highest- $p_{\mathrm{T}}$ jets in the event and $\ell_{a, b, c}$ are the three lepton candidates. The constraints were defined as follows: $m_{t}=172.5 \mathrm{GeV}, m_{W}=80.4 \mathrm{GeV}, m_{Z}=91.2 \mathrm{GeV}$. The widths were determined from the mass resolution of each decay mode in the MC simulation, and set to $\sigma_{t}=14 \mathrm{GeV}, \sigma_{W}=10 \mathrm{GeV}$ and $\sigma_{Z}=3 \mathrm{GeV}$. The transverse momentum of the neutrino was set equal to $E_{\mathrm{T}}^{\text {miss }}$, and all jet and lepton assignments were tried, subject to the requirement that the $Z$ candidate be built from same-flavour oppositecharge leptons. Any opposite-charge ID lepton-TL pair can be used as leptons from the $Z$-boson decay, because the TL is assumed to be the same flavour as the ID lepton. No $b$-jet identification was used in the reconstruction of the event kinematics. For each assignment, the value of $p_{z}^{\nu}$ was defined to be that which gave the minimum $\chi^{2}$. From all combinations, the one with the smallest $\chi^{2}$ was chosen along with the corresponding $p_{z}^{\nu}$ value. Events were rejected unless the reconstructed top-quark masses were within $40 \mathrm{GeV}$ of $m_{t}$, the reconstructed $W$-boson mass was within $30 \mathrm{GeV}$ of $m_{W}$, and the reconstructed $Z$-boson 
mass was within $15 \mathrm{GeV}$ of $m_{Z}$. The effect of these mass cuts on the fake-TL background expectation was derived from simulation by measuring the fraction of simulated 2ID+TL background events with fake TLs that pass the $\chi^{2}$ mass cuts. This fraction is $(31 \pm 10) \%$. Of the events that pass all other event selection requirements, $38 \%$ (29\%) of the 3ID (2ID+TL) events pass the $\chi^{2}$ mass cuts. The efficiency for FCNC MC events to pass the $\chi^{2}$-based mass cuts is $(79 \pm 2) \%((66 \pm 2) \%)$, while for background MC events it is only $(47 \pm 7) \%$ $((33 \pm 10) \%)$ for 3ID (2ID+TL) events.

The signal efficiency for $t \bar{t} \rightarrow W b Z q$, after all selection requirements, was determined using the TopReX sample described in section 3 and is shown in table 1.

\section{Background evaluation}

Backgrounds to this search can be divided into two categories: those with three real leptons and those with at least one fake lepton. Backgrounds with three real leptons arise from diboson ( $W Z$ and $Z Z$ ) production with additional jets, and were evaluated using the MC samples described in section 3.2. In the case of $W Z$ production, the required $E_{\mathrm{T}}^{\text {miss }}$ comes from the neutrino from the leptonic $W$-boson decay. Events from $Z Z$ decays can enter the signal region in several ways; the dominant modes are four-lepton decays with one lepton not reconstructed, giving apparent $E_{\mathrm{T}}^{\text {miss }}$, and $\tau^{+} \tau^{-}$decays with one $\tau$ decaying to $e$ or $\mu$ and two neutrinos.

The background to 3ID candidate events, in which exactly one of the leptons is a fake lepton, was evaluated using a combination of data and MC samples. The dominant contribution in this category comes from $Z+$ jets events, with a leptonic $Z$ decay, in which one of the jets was misidentified as a third lepton. To evaluate this background a datadriven (DD) method was used. This method uses a control region $(\mathrm{CR})$ in the $\left(E_{\mathrm{T}}^{\mathrm{miss}}, m_{\ell \ell}\right)$ plane by selecting events with exactly two opposite-charge electrons or muons (no third ID lepton is allowed) and $\left|91.2 \mathrm{GeV}-m_{\ell \ell}^{\text {reco }}\right|<15 \mathrm{GeV}$ in six different $E_{\mathrm{T}}^{\text {miss }}$ bins from $0 \mathrm{GeV}$ to $\geq 50 \mathrm{GeV}$. The $Z+$ jets estimate in each $E_{\mathrm{T}}^{\text {miss }}$ bin is then given by:

$$
\left[N_{Z+\text { jets }}^{\text {Data }}\right]_{\mathrm{SR}}=\left[\frac{N^{\text {Data }}-N_{\text {Other backgrounds }}^{\mathrm{MC}}}{N_{Z+\text { jets }}^{\mathrm{MC}}}\right]_{\mathrm{CR}} \cdot\left[N_{Z+\text { jets }}^{\mathrm{MC}}\right]_{\mathrm{SR}} .
$$

For each $E_{\mathrm{T}}^{\text {miss }}$ bin considered, the corresponding background-subtracted data/simulation ratio in the CR was applied to the simulated $Z+$ jets background in the signal regions (SR), in order to evaluate the expected number of $Z+$ jets events in the data. Due to the small $\mathrm{MC}$ event sample after the final selection, the $Z+$ jets background was evaluated using a loosened lepton selection and a multiplicative rejection factor to account for the loosened selection. The isolation requirement on electron candidates was raised from $3.5 \mathrm{GeV}$ to $6 \mathrm{GeV}$, and the isolation requirement on muon candidates was removed altogether. A multiplicative rejection factor of $0.063 \pm 0.013$, corresponding to the MC probability for events with loose leptons to pass the SR lepton criteria, was applied to the final result. The remaining backgrounds with one fake lepton (dileptonic $t \bar{t}, W t$-channel single-top and $W W$ production) were evaluated using Monte Carlo simulation samples, described in section 3, 
and the loose lepton selection and multiplicative factor above. Different DD methods and cross-checks for the one-fake-lepton background were performed. These include the matrix method [70], relaxation of $E_{\mathrm{T}}^{\text {miss }}$ or lepton quality requirements, and MC simulation with fake-rate factors measured from data. These alternative methods, although statistically limited, agree with the reference $\mathrm{DD}+\mathrm{MC}$ method used.

A DD method was developed to evaluate the contribution to 3ID events from multijet, $W+$ jets, single-top and $t \bar{t}$ single-lepton decay events, in which two or three jets were reconstructed as leptons ( $2+3$ fake leptons). Due to the requirement that two leptons should have the same flavour and opposite charges, the yield from these backgrounds can be extrapolated from the number of observed data events with three leptons of any flavour $(e$ or $\mu$ ), but with the same charge. Taking into account the possible charge and flavour combinations, there are 36 combinations of three leptons, in which two have the same flavour and opposite charges, and 16 combinations of three leptons with the same charge. The extrapolation factor is thus $f=36 / 16=2.25$. No data event passed the selection after requiring three leptons with the same charge. The uncertainties in the DD backgrounds were determined using the Feldman-Cousins upper interval for a $68 \%$ C.L. [71] with no observed events (with the uncertainties multiplied by 2.25 for the $2+3$ fake leptons sample). Since no events were selected with three leptons of the same charge, a multiplicative factor of $0.071 \pm 0.018$, to account for the final requirements of at least two jets with $p_{\mathrm{T}}>25 \mathrm{GeV}$ and $E_{\mathrm{T}}^{\text {miss }}>20 \mathrm{GeV}$ was evaluated using $\mathrm{MC}$ samples and applied to the uncertainty estimate.

In 2ID+TL events the dominant background contribution comes from events with a fake TL. The background contribution from such events was evaluated with the same technique used in ref. [64]: the probability of a jet being reconstructed as a track lepton was determined from a $\gamma+$ jets data sample selected with photon triggers, and parameterised in a 'fake matrix' as a function of jet $p_{\mathrm{T}}$ and the number of primary vertices in the event, $N_{\text {vtx }}$. The number of primary vertices was needed in the parameterisation because the fake probability is sensitive to pile-up. The fake matrix was applied to a 'parent sample' selected with all of the signal region requirements with the exception of the three leptons. Instead, two ID leptons were required. Fake probabilities from the matrix were summed for each jet in the parent sample, according to its $p_{\mathrm{T}}$ and $N_{\mathrm{vtx}}$ for each event. The resulting sum is the fake TL background contribution. Because of the $b$-tag requirement in events with a TL, a $b$-tagged jet was allowed to contribute to the sum only if there was another $b$-tagged jet in the event. This accounts for the fact that if a jet produced a fake TL, the remaining jet would be removed by the lepton-jet overlap removal described in section 4 . For the same reason, events with three or more jets were used to predict the number of fake TLs in events with two or more jets. The signal region required a $Z$-boson candidate, i.e. an opposite-charge, same-flavour lepton pair. Therefore the parent sample with two ID leptons provides three different cases:

1. Opposite-charge ID leptons

2. Two positively-charged ID leptons

3. Two negatively-charged ID leptons 


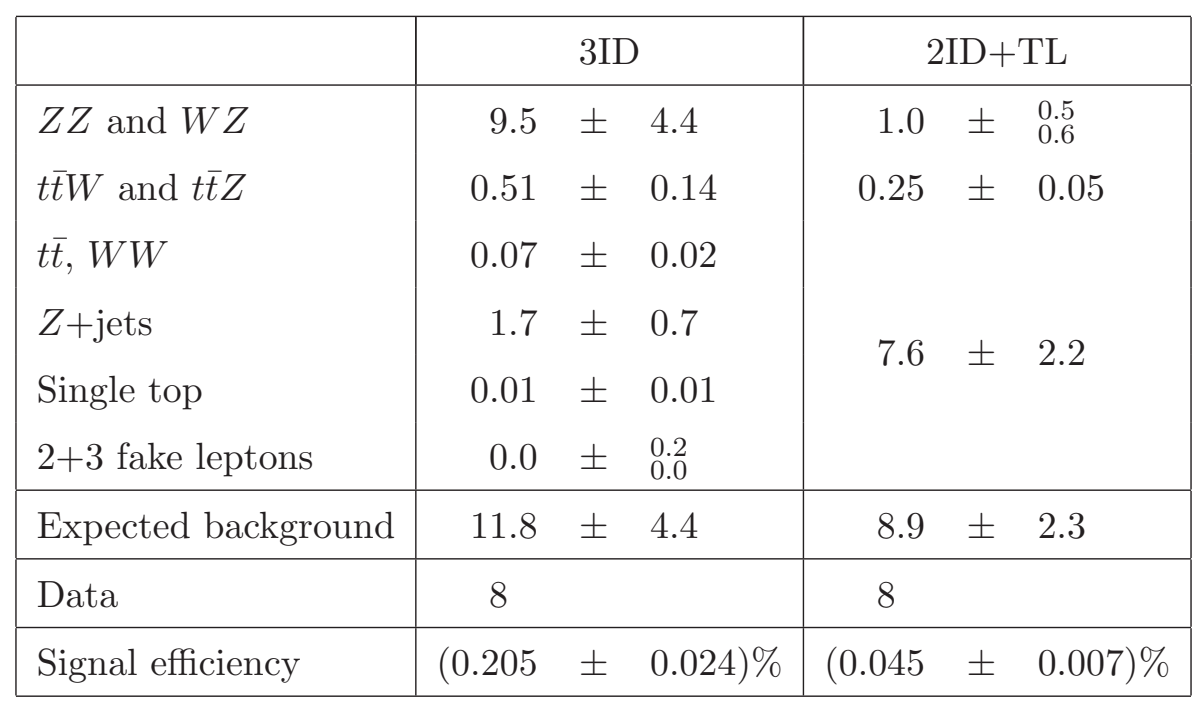

Table 1. Expected number of background events, number of selected data events and signal efficiency (normalized to all decays of the $W$ and $Z$ bosons), after the final event selection. The $t \bar{t}$ backgrounds correspond to SM decays of the top quarks. The third entry in the 2ID+TL column corresponds to the fake TL background and includes all sources of events in the left-hand column except $Z Z, W Z, t \bar{t} W$ and $t \bar{t} Z$.

In case 1 , the fake TL was allowed to have either charge. In case 2 the fake TL was required to be negatively charged, and in case 3 positively charged. Three different fake matrices were constructed to account for these three cases, one in which both charges are used, and one with only negatively or positively charged TLs. When a TL and an oppositely-charged ID lepton had an invariant mass consistent with arising from a $Z$ boson, the same-flavour requirement was automatically satisfied because the TL is taken to have the same flavour as the ID lepton. The parent sample with two ID leptons contains all sources of backgrounds that can enter the signal region with a fake TL, including those with one or two fake ID leptons. Thus the procedure predicts the full background contribution with a fake TL. A small contribution ( $2 \%$ of the total), evaluated from the MC simulation, was included to account for events with a 'real' TL and a fake ID lepton.

A summary of expected backgrounds and selected data events in both the 3ID lepton and $2 \mathrm{ID}+\mathrm{TL}$ samples is shown in table 1 . Figure 2 shows good agreement in the $E_{\mathrm{T}}^{\mathrm{miss}}$ distributions of data and expected backgrounds in background-dominated control regions for the 3ID and 2ID+TL selections.

Figure 3 shows the reconstructed candidate $Z$-boson and top-quark masses, $m_{l l}$ and $m_{l l q}$ respectively, for the FCNC decay hypothesis in the selected candidate events, for both the 3ID and 2ID+TL data, compared with the expectations from SM backgrounds and the FCNC signal.

\section{Systematic uncertainties}

A number of systematic uncertainties can influence the expected number of signal and/or background events. The effect of each source of systematic uncertainty was studied by 


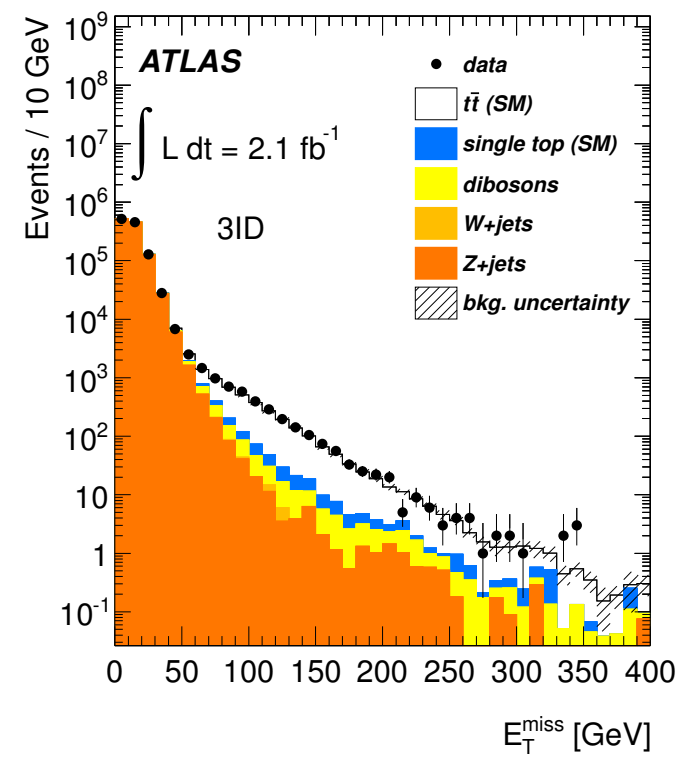

(a)

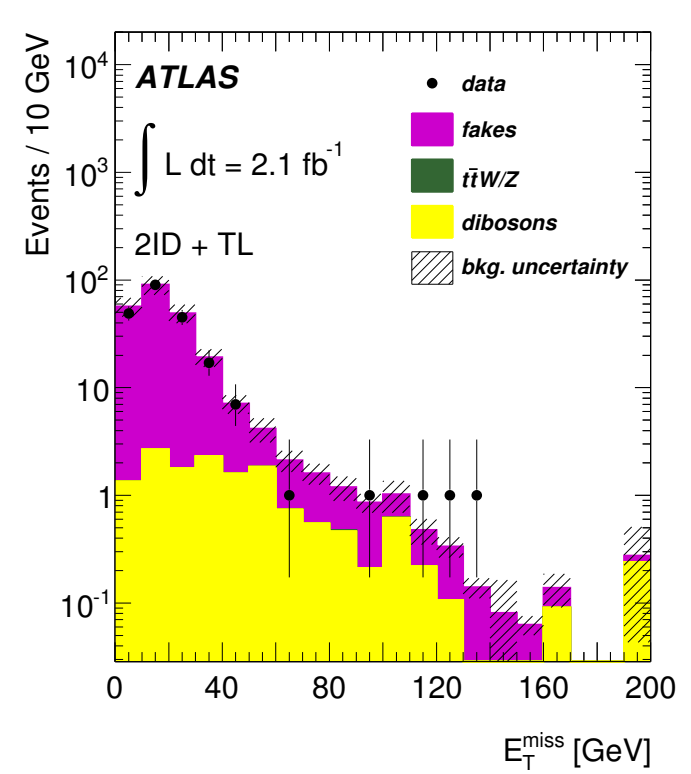

(b)

Figure 2. $E_{\mathrm{T}}^{\text {miss }}$ distributions in control regions for the (a) 3ID and (b) 2ID+TL events. The 3ID control region is defined by two same-flavor, opposite-charge leptons with an invariant mass within $15 \mathrm{GeV}$ of $M_{Z}=91.2 \mathrm{GeV}$. The 2ID+TL control region is defined by two ID leptons, one TL and exactly one jet. In the 2ID+TL case, backgrounds from $t \bar{t}$ and $W$ and $Z$ plus jets are included in the 'fakes' contribution.

independently varying the corresponding central value by the estimated uncertainty. For each variation, the total number of expected background events and the signal efficiencies were compared with the reference values.

The measurement of the integrated luminosity has a total uncertainty of $3.7 \%$ [40, 41]. This uncertainty was considered in the analyses by changing the normalisations of the backgrounds evaluated from MC simulation. Uncertainties associated with the energy scale of light-quark jets and $b$-jets were studied as a function of the jet transverse momentum and pseudorapidity. These uncertainties, including the effects of pile-up, are in the range 6-10\% [67]. The effects of the jet reconstruction efficiency uncertainty were studied by randomly removing about $2 \%$ of jets from the events. The effect of potential jet resolution mis-modelling in the MC simulation was evaluated by additional smearing of the reconstructed jet energies within the uncertainties. In each case, the difference with respect to the nominal simulation was considered as the systematic uncertainty. The uncertainties due to MC modelling of the lepton trigger, reconstruction and selection efficiencies, and $b$-tagging efficiency, were taken into account by re-computing the predicted event yields and signal acceptance using the corresponding systematic shift. The momentum of the lepton in simulation was rescaled and smeared to correct for scale and resolution disagreements between simulated and observed data. The systematic uncertainty associated with the modelling of the momentum scale and resolution was evaluated by shifting the momentum scale and changing the smearing factors. Changes applied to electrons, muons and jets 


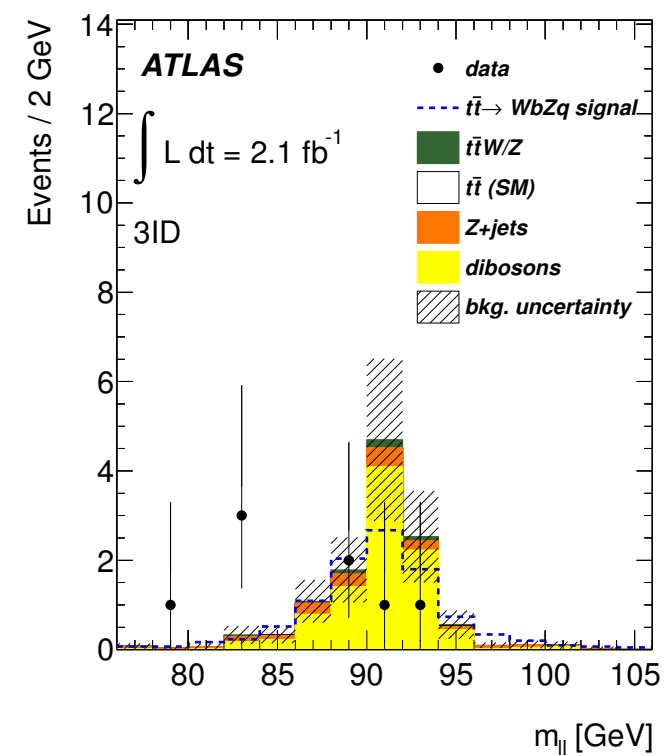

(a)

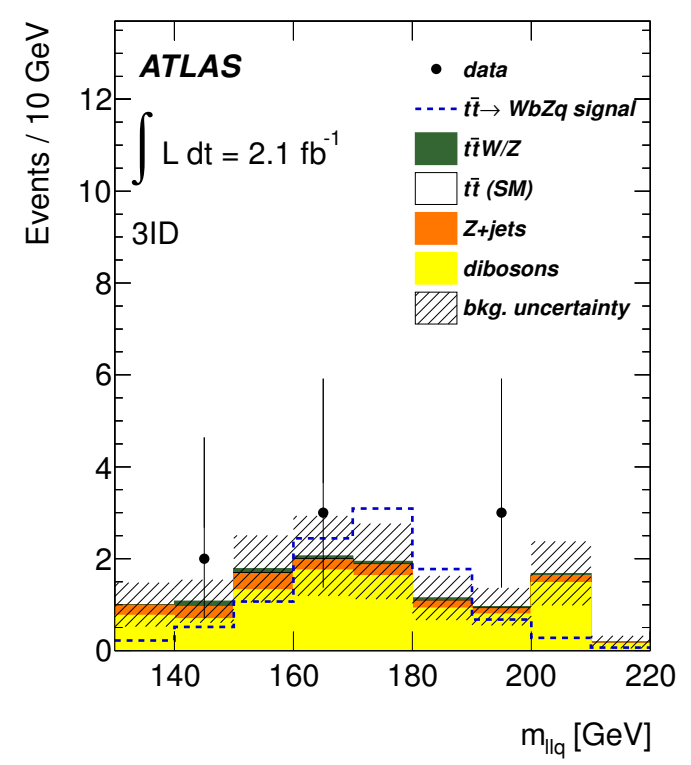

(c)

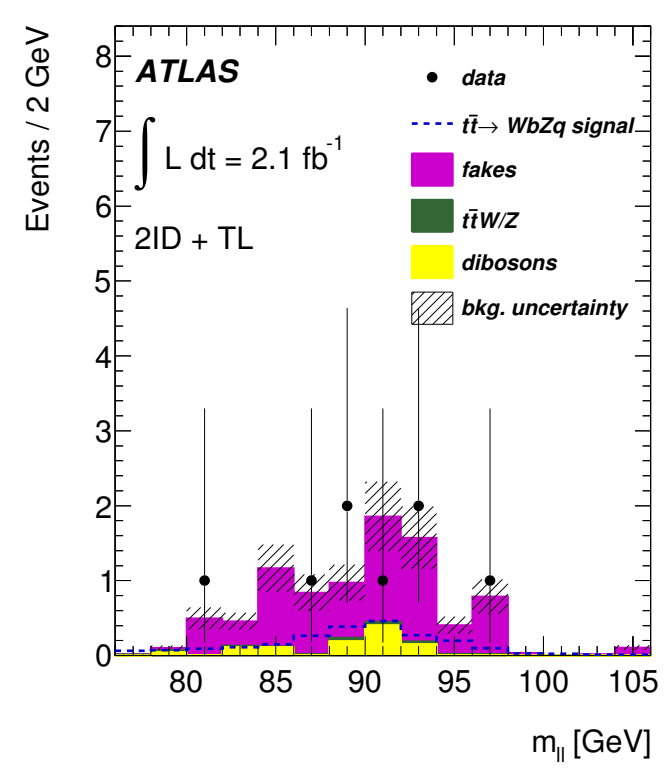

(b)

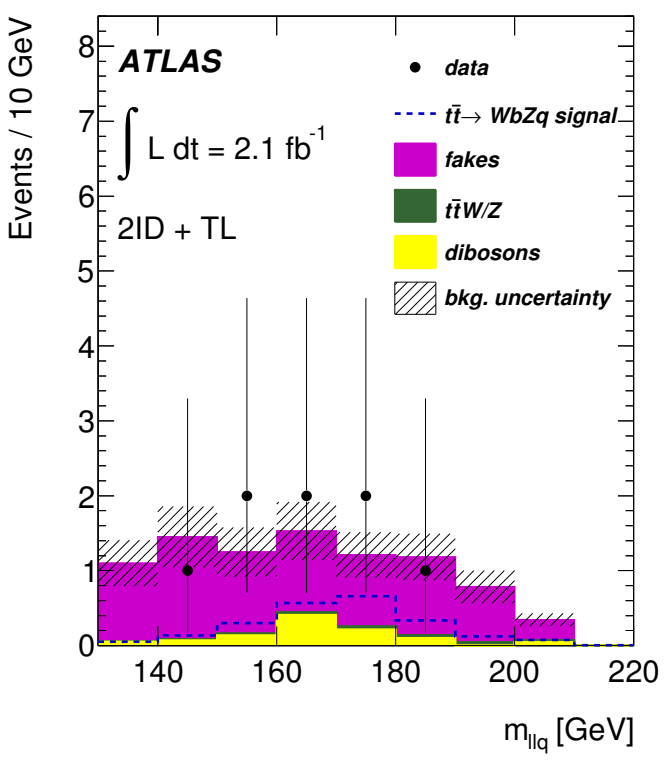

(d)

Figure 3. Expected and observed $Z$-boson and top-quark mass distributions for the FCNC decay hypothesis in the 3ID ((a) \& (c)) and 2ID+TL ((b) \& (d)) candidate events after all selection requirements. The $t \bar{t} \rightarrow W b Z q$ distributions are normalized to the observed limit in each channel.

were propagated to $E_{\mathrm{T}}^{\text {miss }}$. Uncertainties related to $E_{\mathrm{T}}^{\text {miss }}$ were also studied: the effect of the energy in the calorimeter not associated with the above objects, and of low momentum $\left(7 \mathrm{GeV}<p_{\mathrm{T}}<20 \mathrm{GeV}\right)$ jets, was studied, as well as the uncertainty due to modelling of pile-up. The effect of a hardware failure in the electromagnetic calorimeter was also considered as a systematic uncertainty (LAr readout problem) and evaluated by varying 
the jet thresholds used for removing events with jets directed at the dead region. The effects of ISR/FSR and top-quark mass uncertainties were evaluated using the MC samples described in section 3. The effect of uncertainties in the PDF used for signal generation was evaluated by comparing the signal acceptance using MSTW2008LO with that from MRST2007 LO* PDFs. The systematic uncertainties related to the $Z Z$ and $W Z$ simulation modelling were estimated using the Berends-Giele scaling $[72,73]$ with an uncertainty of $24 \%$ per jet, added in quadrature. An uncertainty of $4 \%$ was included for the 0 -jet bin. The $Z Z$ and $W Z$ cross sections were varied by their theoretical uncertainty of $5 \%$ [74]. The uncertainties on the $Z+$ jets normalisations were derived using a data-driven method. In the 2ID+TL channel, where $b$-tagging was used, a systematic uncertainty associated with the heavy-flavour content of $W Z+$ jets and $Z Z+$ jets is included. This was evaluated by comparing ALPGEN and MC@NLO, and is small because the dominant source of $b$-tags in these events comes from mis-tags of light-quark jets, with a secondary component from charm jets.

The dominant source of systematic uncertainty for the 3ID channel is the $Z Z$ and $W Z$ simulation modelling. The other sources have effects at most of the same magnitude as the statistical uncertainty. The dominant uncertainty in the 2ID+TL channel is the systematic uncertainty on the fake-TL prediction, because $90 \%$ of the expected background arises from this source. This was determined to be $20 \%$ by comparing predicted and observed events with TLs in control regions dominated by fake TLs [64].

The resulting uncertainties for the backgrounds and signal acceptance are shown in table 2. Because almost $90 \%$ of the $2 \mathrm{ID}+\mathrm{TL}$ background evaluation is data-driven, the 2ID+TL analysis has a smaller relative background systematic uncertainties in most categories, compared to the 3ID analysis.

\section{Limit evaluation}

Good agreement between data and expected background yields was observed, as shown in table 1. No evidence for the $t \rightarrow Z q$ decay mode was found and $95 \%$ C.L. upper limits on the number of signal events were derived using the modified frequentist $\left(\mathrm{CL}_{\mathrm{s}}\right)$ likelihood method [75, 76]. The statistical fluctuations of the pseudo-experiments were performed using Poisson distributions. All statistical and systematic uncertainties of the expected backgrounds and signal efficiencies were taken into account, as described in section 7 and were implemented assuming Gaussian distributions [75]. The systematic uncertainties of the $Z Z, W Z$ and signal acceptance were considered to be fully correlated between the 3ID and 2ID+TL channels, while all other sources of uncertainties (statistical or systematic) were considered uncorrelated. The limits on the number of signal events were converted into upper limits on the corresponding BRs using the approximate NNLO calculation, and its uncertainty, for the $t \bar{t}$ cross section $\left(\sigma_{t \bar{t}}=165_{-16}^{+11} \mathrm{pb}\right)$ [59], and constraining $\mathrm{BR}(t \rightarrow$ $W b)=1-\mathrm{BR}(t \rightarrow Z q)$. The observed 95\% C.L. upper limit on the FCNC $t \rightarrow Z q \mathrm{BR}$ is $0.81 \%(3.2 \%)$ taking the 3ID (2ID+TL) events and background evaluation alone, and $0.73 \%$ when the 3ID and 2ID+TL results are combined. Table 3 shows the observed and expected limits in the absence of signal for the 3ID and 2ID+TL channels, as well as for the combination. Also shown are the $\pm 1 \sigma$ expected limits. 


\begin{tabular}{|lrr|rr|}
\hline & \multicolumn{2}{c}{ 3ID } & \multicolumn{2}{c|}{ 2ID+TL } \\
\hline Source & Background & Signal & Background & Signal \\
\hline Luminosity & $4 \%$ & $4 \%$ & $<1 \%$ & $4 \%$ \\
\hline Electron trigger & $4 \%$ & $1 \%$ & $<1 \%$ & $<1 \%$ \\
Electron reconstruction modelling & $10 \%$ & $3 \%$ & $<1 \%$ & $2 \%$ \\
\hline Muon trigger & $3 \%$ & $1 \%$ & $<1 \%$ & $<1 \%$ \\
Muon reconstruction modelling & $7 \%$ & $1 \%$ & $<1 \%$ & $1 \%$ \\
TL reconstruction modelling & - & - & $2 \%$ & $1 \%$ \\
\hline Jet energy scale & $11 \%$ & $1 \%$ & $1 \%$ & $1 \%$ \\
Jet reconstruction efficiency & $5 \%$ & $2 \%$ & $<1 \%$ & $<1 \%$ \\
Jet energy resolution & $1 \%$ & $3 \%$ & $1 \%$ & $4 \%$ \\
\hline$E_{\mathrm{T}}^{\text {miss } \text { modelling }}$ & $4 \%$ & $1 \%$ & $<1 \%$ & $<1 \%$ \\
LAr readout problem & $3 \%$ & $1 \%$ & $<1 \%$ & $1 \%$ \\
Pile-up & $4 \%$ & $<1 \%$ & $<1 \%$ & $<1 \%$ \\
$b$-tagging & - & - & $1 \%$ & $6 \%$ \\
\hline Top quark mass & $<1 \%$ & $2 \%$ & - & $3 \%$ \\
$\sigma_{t \bar{t}}$ & $<1 \%$ & $8 \%$ & - & $8 \%$ \\
ISR/FSR & $<1 \%$ & $3 \%$ & - & $6 \%$ \\
PDFs & - & $3 \%$ & - & $3 \%$ \\
\hline$Z Z$ and $W Z$ shape & $33 \%$ & - & $5 \%$ & - \\
$Z Z$ and $W Z$ cross section & $4 \%$ & - & $<1 \%$ & - \\
$Z Z$ and $W Z$ heavy-flavour content & - & - & $<1 \%$ & - \\
\hline Fake leptons & $1 \%$ & - & $17 \%$ & - \\
Total & $38 \%$ & $12 \%$ & $18 \%$ & $15 \%$ \\
\hline
\end{tabular}

Table 2. Relative changes in the expected number of background events and signal yield for different sources of systematic uncertainties. The contributions from the $Z Z$ and $W Z$ event generator apply only to the simulated background samples.

\begin{tabular}{|lcccc|}
\hline channel & observed & $(-1 \sigma)$ & expected & $(+1 \sigma)$ \\
\hline 3ID & $0.81 \%$ & $0.63 \%$ & $0.95 \%$ & $1.4 \%$ \\
2ID+TL & $3.2 \%$ & $2.15 \%$ & $3.31 \%$ & $4.9 \%$ \\
Combination & $0.73 \%$ & $0.61 \%$ & $0.93 \%$ & $1.4 \%$ \\
\hline
\end{tabular}

Table 3. The expected and observed 95\% C.L. upper limits on the FCNC top quark decay $t \rightarrow Z q$ BR. The $\pm 1 \sigma$ expected limits include both statistical and systematic uncertainties.

\section{Conclusions}

A search for FCNC decays of top quarks produced in pairs was performed using data collected by the ATLAS experiment at a centre-of-mass energy of $\sqrt{s}=7 \mathrm{TeV}$ and corresponding to an integrated luminosity of $2.1 \mathrm{fb}^{-1}$. The search for the $t \rightarrow Z q$ decay mode was performed by studying top-quark pair production with one top quark decaying according to the Standard Model and the other according to the FCNC $(t \bar{t} \rightarrow W b Z q)$. 
No evidence for such a signal was found. An observed limit at 95\% C.L. on the $t \rightarrow Z q$ FCNC top-quark decay branching fraction was set at $\mathrm{BR}(t \rightarrow Z q)<0.73 \%$, assuming $\mathrm{BR}(t \rightarrow W b)+\mathrm{BR}(t \rightarrow Z q)=1$. The observed limit is compatible with the expected sensitivity, assuming that the data are described correctly by the Standard Model, of $\mathrm{BR}(t \rightarrow Z q)<0.93 \%$.

\section{Acknowledgments}

We thank CERN for the very successful operation of the LHC, as well as the support staff from our institutions without whom ATLAS could not be operated efficiently.

We acknowledge the support of ANPCyT, Argentina; YerPhI, Armenia; ARC, Australia; BMWF, Austria; ANAS, Azerbaijan; SSTC, Belarus; CNPq and FAPESP, Brazil; NSERC, NRC and CFI, Canada; CERN; CONICYT, Chile; CAS, MOST and NSFC, China; COLCIENCIAS, Colombia; MSMT CR, MPO CR and VSC CR, Czech Republic; DNRF, DNSRC and Lundbeck Foundation, Denmark; EPLANET and ERC, European Union; IN2P3-CNRS, CEA-DSM/IRFU, France; GNAS, Georgia; BMBF, DFG, HGF, MPG and AvH Foundation, Germany; GSRT, Greece; ISF, MINERVA, GIF, DIP and Benoziyo Center, Israel; INFN, Italy; MEXT and JSPS, Japan; CNRST, Morocco; FOM and NWO, Netherlands; RCN, Norway; MNiSW, Poland; GRICES and FCT, Portugal; MERYS (MECTS), Romania; MES of Russia and ROSATOM, Russian Federation; JINR; MSTD, Serbia; MSSR, Slovakia; ARRS and MVZT, Slovenia; DST/NRF, South Africa; MICINN, Spain; SRC and Wallenberg Foundation, Sweden; SER, SNSF and Cantons of Bern and Geneva, Switzerland; NSC, Taiwan; TAEK, Turkey; STFC, the Royal Society and Leverhulme Trust, United Kingdom; DOE and NSF, United States of America.

The crucial computing support from all WLCG partners is acknowledged gratefully, in particular from CERN and the ATLAS Tier-1 facilities at TRIUMF (Canada), NDGF (Denmark, Norway, Sweden), CC-IN2P3 (France), KIT/GridKA (Germany), INFN-CNAF (Italy), NL-T1 (Netherlands), PIC (Spain), ASGC (Taiwan), RAL (UK) and BNL (USA) and in the Tier-2 facilities worldwide.

Open Access. This article is distributed under the terms of the Creative Commons Attribution License which permits any use, distribution and reproduction in any medium, provided the original author(s) and source are credited.

\section{References}

[1] Tevatron Electroweak Working Group, CDF and D0 collaboration, Combination of CDF and DO results on the mass of the top quark using up to $5.8 \mathrm{fb}^{-1}$ of data, arXiv:1107.5255 [INSPIRE].

[2] S. Glashow, J. Iliopoulos and L. Maiani, Weak interactions with lepton-hadron symmetry, Phys. Rev. D 2 (1970) 1285 [INSPIRE].

[3] J. Aguilar-Saavedra and B. Nobre, Rare top decays $t \rightarrow c \gamma, t \rightarrow c g$ and CKM unitarity, Phys. Lett. B 553 (2003) 251 [hep-ph/0210360] [INSPIRE]. 
[4] F. del Aguila, J.A. Aguilar-Saavedra and R. Miquel, Constraints on top couplings in models with exotic quarks, Phys. Rev. Lett. 82 (1999) 1628 [hep-ph/9808400] [INSPIRE].

[5] J.A. Aguilar-Saavedra, Effects of mixing with quark singlets, Phys. Rev. D 67 (2003) 035003 [Erratum ibid. D 69 (2004) 099901] [hep-ph/0210112] [INSPIRE].

[6] T.P. Cheng and M. Sher, Mass matrix ansatz and flavor nonconservation in models with multiple Higgs doublets, Phys. Rev. D 35 (1987) 3484 [INSPIRE].

[7] B. Grzadkowski, J.F. Gunion and P. Krawczyk, Neutral current flavor changing decays for the $Z$ boson and the top quark in two Higgs doublet models, Phys. Lett. B 268 (1991) 106 [INSPIRE].

[8] M.E. Luke and M.J. Savage, Flavor changing neutral currents in the Higgs sector and rare top decays, Phys. Lett. B 307 (1993) 387 [hep-ph/9303249] [INSPIRE].

[9] D. Atwood, L. Reina and A. Soni, Probing flavor changing top-charm-scalar interactions in $e^{+} e^{-}$collisions, Phys. Rev. D 53 (1996) 1199 [hep-ph/9506243] [INSPIRE].

[10] D. Atwood, L. Reina and A. Soni, Phenomenology of two Higgs doublet models with flavor changing neutral currents, Phys. Rev. D 55 (1997) 3156 [hep-ph/9609279] [INSPIRE].

[11] S. Bejar, J. Guasch and J. Solà, Loop induced flavor changing neutral decays of the top quark in a general two Higgs doublet model, Nucl. Phys. B 600 (2001) 21 [hep-ph/0011091] [INSPIRE].

[12] C.S. Li, R.J. Oakes and J.M. Yang, Rare decay of the top quark in the minimal supersymmetric model, Phys. Rev. D 49 (1994) 293 [Erratum ibid. D 56 (1997) 3156] [INSPIRE].

[13] G. de Divitiis, R. Petronzio and L. Silvestrini, Flavor changing top decays in supersymmetric extensions of the standard model, Nucl. Phys. B 504 (1997) 45 [hep-ph/9704244] [INSPIRE].

[14] J.L. Lopez, D.V. Nanopoulos and R. Rangarajan, New supersymmetric contributions to $t \rightarrow c V$, Phys. Rev. D 56 (1997) 3100 [hep-ph/9702350] [INSPIRE].

[15] J. Guasch and J. Solà, FCNC top quark decays: a door to SUSY physics in high luminosity colliders?, Nucl. Phys. B 562 (1999) 3 [hep-ph/9906268] [INSPIRE].

[16] D. Delepine and S. Khalil, Top flavor violating decays in general supersymmetric models, Phys. Lett. B 599 (2004) 62 [hep-ph/0406264] [INSPIRE].

[17] J.J. Liu, C.S. Li, L.L. Yang and L.G. Jin, $t \rightarrow c V$ via SUSY FCNC couplings in the unconstrained MSSM, Phys. Lett. B 599 (2004) 92 [hep-ph/0406155] [INSPIRE].

[18] J. Cao et al., SUSY-induced FCNC top-quark processes at the Large Hadron Collider, Phys. Rev. D 75 (2007) 075021 [hep-ph/0702264] [INSPIRE].

[19] J.M. Yang, B.-L. Young and X. Zhang, Flavor changing top quark decays in R parity violating SUSY, Phys. Rev. D 58 (1998) 055001 [hep-ph/9705341] [inSPIRE].

[20] G.-R. Lu, F.-R. Yin, X.-L. Wang and L.-D. Wan, The rare top quark decays $t \rightarrow c \mathrm{~V}$ in the topcolor assisted technicolor model, Phys. Rev. D 68 (2003) 015002 [hep-ph/0303122] [INSPIRE].

[21] K. Agashe, G. Perez and A. Soni, Flavor structure of warped extra dimension models, Phys. Rev. D 71 (2005) 016002 [hep-ph/0408134] [INSPIRE].

[22] K. Agashe, G. Perez and A. Soni, Collider signals of top quark flavor violation from a warped extra dimension, Phys. Rev. D 75 (2007) 015002 [hep-ph/0606293] [INSPIRE]. 
[23] CDF collaboration, F. Abe et al., Search for flavor-changing neutral current decays of the top quark in $p \bar{p}$ collisions at $\sqrt{s}=1.8$ TeV, Phys. Rev. Lett. 80 (1998) 2525 [INSPIRE].

[24] CDF collaboration, T. Aaltonen et al., Search for the flavor changing neutral current decay $t \rightarrow Z q$ in $p \bar{p}$ collisions at $\sqrt{s}=1.96$ TeV, Phys. Rev. Lett. 101 (2008) 192002 [arXiv:0805.2109] [INSPIRE].

[25] CDF collaboration, T. Aaltonen et al., Search for top-quark production via flavor-changing neutral currents in $W+1$ jet events at CDF, Phys. Rev. Lett. 102 (2009) 151801 [arXiv:0812.3400] [INSPIRE].

[26] D0 collaboration, V.M. Abazov et al., Search for flavor changing neutral currents via quark-gluon couplings in single top quark production using $2.3 \mathrm{fb}^{-1}$ of $p \bar{p}$ collisions, Phys. Lett. B 693 (2010) 81 [arXiv:1006.3575] [INSPIRE].

[27] D0 collaboration, V.M. Abazov et al., Search for flavor changing neutral currents in decays of top quarks, Phys. Lett. B 701 (2011) 313 [arXiv:1103.4574] [INSPIRE].

[28] ALEPH collaboration, A. Heister et al., Search for single top production in $e^{+} e^{-}$collisions at $\sqrt{s}$ up to $209 \mathrm{GeV}$, Phys. Lett. B 543 (2002) 173 [hep-ex/0206070] [INSPIRE].

[29] DELPHI collaboration, J. Abdallah et al., Search for single top production via FCNC at LEP at $\sqrt{s}=189$ GeV to 208 GeV, Phys. Lett. B 590 (2004) 21 [hep-ex/0404014] [INSPIRE].

[30] OPAL collaboration, G. Abbiendi et al., Search for single top quark production at LEP-2, Phys. Lett. B 521 (2001) 181 [hep-ex/0110009] [INSPIRE].

[31] L3 collaboration, P. Achard et al., Search for single top production at LEP, Phys. Lett. B 549 (2002) 290 [hep-ex/0210041] [INSPIRE].

[32] LEP ExотісA WG, Search for single top production via flavour changing neutral currents: preliminary combined results of the LEP experiments, LEP Exotica WG 2001-01 (2001).

[33] M. Beneke et al., Top quark physics, hep-ph/0003033 [INSPIRE].

[34] ZEUS collaboration, H. Abramowicz et al., Search for single-top production in ep collisions at HERA, Phys. Lett. B 708 (2012) 27 [arXiv:1111.3901] [INSPIRE].

[35] H1 collaboration, F. Aaron et al., Search for single top quark production at HERA, Phys. Lett. B 678 (2009) 450 [arXiv:0904.3876] [INSPIRE].

[36] H1 collaboration, A. Aktas et al., Search for single top quark production in ep collisions at HERA, Eur. Phys. J. C 33 (2004) 9 [hep-ex/0310032] [INSPIRE].

[37] ZEUS collaboration, S. Chekanov et al., Search for single top production in ep collisions at HERA, Phys. Lett. B 559 (2003) 153 [hep-ex/0302010] [INSPIRE].

[38] ATLAS collaboration, G. Aad et al., Search for FCNC single top-quark production at $\sqrt{s}=7 \mathrm{TeV}$ with the ATLAS detector, Phys. Lett. B 712 (2012) 351 [arXiv:1203.0529] [INSPIRE].

[39] ATLAS collaboration, G. Aad et al., The ATLAS experiment at the CERN Large Hadron Collider, 2008 JINST 3 S08003 [INSPIRE].

[40] ATLAS collaboration, G. Aad et al., Luminosity determination in pp collisions at $\sqrt{s}=7$ TeV using the ATLAS detector at the LHC, Eur. Phys. J. C 71 (2011) 1630 [arXiv:1101.2185] [INSPIRE]. 
[41] ATLAS collaboration, Luminosity determination in pp collisions at $\sqrt{s}=7 \mathrm{TeV}$ using the ATLAS Detector in 2011, ATLAS-CONF-2011-116 (2011).

[42] GEANT4 collaboration, S. Agostinelli et al., GEANT4: a simulation toolkit, Nucl. Instrum. Meth. A 506 (2003) 250 [inSPIRE].

[43] ATLAS collaboration, G. Aad et al., The ATLAS simulation Infrastructure, Eur. Phys. J. C 70 (2010) 823 [arXiv: 1005.4568] [InSPIRE].

[44] S. Slabospitsky and L. Sonnenschein, TopReX generator (version 3.25): short manual, Comput. Phys. Commun. 148 (2002) 87 [hep-ph/0201292] [INSPIRE].

[45] A. Sherstnev and R. Thorne, Parton distributions for LO generators, Eur. Phys. J. C 55 (2008) 553 [arXiv:0711.2473] [INSPIRE].

[46] T. Sjöstrand, S. Mrenna and P.Z. Skands, PYTHIA 6.4 physics and manual, JHEP 05 (2006) 026 [hep-ph/0603175] [INSPIRE].

[47] B.P. Kersevan and E. Richter-Was, The Monte Carlo event generator AcerMC version 2.0 with interfaces to PYTHIA 6.2 and HERWIG 6.5, hep-ph/0405247 [INSPIRE].

[48] P.Z. Skands, Tuning Monte Carlo generators: the Perugia tunes, Phys. Rev. D 82 (2010) 074018 [arXiv: 1005.3457] [INSPIRE].

[49] M.L. Mangano, M. Moretti, F. Piccinini, R. Pittau and A.D. Polosa, ALPGEN, a generator for hard multiparton processes in hadronic collisions, JHEP 07 (2003) 001 [hep-ph/0206293] [INSPIRE].

[50] J. Pumplin et al., New generation of parton distributions with uncertainties from global QCD analysis, JHEP 07 (2002) 012 [hep-ph/0201195] [INSPIRE].

[51] G. Corcella et al., HERWIG 6: an event generator for hadron emission reactions with interfering gluons (including supersymmetric processes), JHEP 01 (2001) 010 [hep-ph/0011363] [INSPIRE].

[52] G. Corcella et al., HERWIG 6.5 release note, hep-ph/0210213 [INSPIRE].

[53] J. Butterworth, J.R. Forshaw and M. Seymour, Multiparton interactions in photoproduction at HERA, Z. Phys. C 72 (1996) 637 [hep-ph/9601371] [INSPIRE].

[54] ATLAS collaboration, First tuning of HERWIG/JIMMY to ATLAS data, PHYS-PUB-2010-014 (2010).

[55] S. Frixione and B.R. Webber, Matching NLO QCD computations and parton shower simulations, JHEP 06 (2002) 029 [hep-ph/0204244] [INSPIRE].

[56] S. Frixione, P. Nason and B.R. Webber, Matching NLO QCD and parton showers in heavy flavor production, JHEP 08 (2003) 007 [hep-ph/0305252] [INSPIRE].

[57] S. Frixione, E. Laenen, P. Motylinski and B.R. Webber, Single-top production in MC@NLO, JHEP 03 (2006) 092 [hep-ph/0512250] [INSPIRE].

[58] P.M. Nadolsky et al., Implications of CTEQ global analysis for collider observables, Phys. Rev. D 78 (2008) 013004 [arXiv:0802.0007] [INSPIRE].

[59] M. Aliev et al., HATHOR: HAdronic Top and Heavy quarks crOss section calculatoR, Comput. Phys. Commun. 182 (2011) 1034 [arXiv:1007.1327] [InSPIRE].

[60] N. Kidonakis, Next-to-next-to-leading-order collinear and soft gluon corrections for t-channel single top quark production, Phys. Rev. D 83 (2011) 091503 [arXiv:1103.2792] [INSPIRE]. 
[61] N. Kidonakis, NNLL resummation for s-channel single top quark production, Phys. Rev. D 81 (2010) 054028 [arXiv: 1001.5034] [InSPIRE].

[62] N. Kidonakis, Two-loop soft anomalous dimensions for single top quark associated production with $a W^{-}$or $H^{-}$, Phys. Rev. D 82 (2010) 054018 [arXiv: 1005.4451] [InSPIRE].

[63] J. Alwall, P. Demin, S. de Visscher, R. Frederix, M. Herquet, et al., MadGraph/MadEvent v4: the new web generation, JHEP 09 (2007) 028 [arXiv:0706.2334] [INSPIRE].

[64] ATLAS collaboration, G. Aad et al., Measurement of the cross section for top-quark pair production in pp collisions at $\sqrt{s}=7 \mathrm{TeV}$ with the ATLAS detector using final states with two high-p $p_{T}$ leptons, JHEP 05 (2012) 059 [arXiv: 1202.4892] [INSPIRE].

[65] ATLAS collaboration, G. Aad et al., Electron performance measurements with the ATLAS detector using the 2010 LHC proton-proton collision data, Eur. Phys. J. C 72 (2012) 1909 [arXiv:1110.3174] [INSPIRE].

[66] M. Cacciari, G.P. Salam and G. Soyez, The anti-kt jet clustering algorithm, JHEP 04 (2008) 063 [arXiv:0802.1189] [INSPIRE].

[67] ATLAS collaboration, G. Aad et al., Jet energy measurement with the ATLAS detector in proton-proton collisions at $\sqrt{s}=7 \mathrm{TeV}$, arXiv:1112.6426 [INSPIRE].

[68] ATLAS collaboration, Commissioning of the ATLAS high-performance b-tagging algorithms in the 7 TeV collision data, ATLAS-CONF-2011-102 (2011).

[69] ATLAS collaboration, G. Aad et al., Performance of missing transverse momentum reconstruction in proton-proton collisions at $7 \mathrm{TeV}$ with ATLAS,

Eur. Phys. J. C 72 (2012) 1844 [arXiv:1108.5602] [INSPIRE].

[70] ATLAS collaboration, G. Aad et al., Measurement of the top quark-pair production cross section with ATLAS in pp collisions at $\sqrt{s}=7$ TeV, Eur. Phys. J. C 71 (2011) 1577 [arXiv: 1012.1792] [INSPIRE].

[71] G.J. Feldman and R.D. Cousins, A unified approach to the classical statistical analysis of small signals, Phys. Rev. D 57 (1998) 3873 [physics/9711021] [INSPIRE].

[72] F.A. Berends, H. Kuijf, B. Tausk and W. Giele, On the production of $a W$ and jets at hadron colliders, Nucl. Phys. B 357 (1991) 32 [inSPIRE].

[73] S. Ellis, R. Kleiss and W.J. Stirling, W's, Z's and Jets, Phys. Lett. B 154 (1985) 435 [INSPIRE].

[74] J.M. Campbell and R.K. Ellis, MCFM for the Tevatron and the LHC, Nucl. Phys. Proc. Suppl. B 205 (2010) 10.

[75] T. Junk, Confidence level computation for combining searches with small statistics, Nucl. Instrum. Meth. A 434 (1999) 435 [hep-ex/9902006] [INSPIRE].

[76] A.L. Read, Modified frequentist analysis of search results (the $C L_{s}$ method), CERN-OPEN-2000-205 (2000). 


\section{The ATLAS collaboration}

G. $\operatorname{Aad}^{48}$, B. Abbott ${ }^{111}$, J. Abdallah ${ }^{11}$, S. Abdel Khalek ${ }^{115}$, A.A. Abdelalim ${ }^{49}$, O. Abdinov ${ }^{10}$, B. Abi ${ }^{112}$, M. Abolins ${ }^{88}$, O.S. AbouZeid ${ }^{158}$, H. Abramowicz ${ }^{153}$, H. Abreu ${ }^{136}$, E. Acerbi ${ }^{89 a, 89 b}$, B.S. Acharya ${ }^{164 a, 164 b}$, L. Adamczyk ${ }^{37}$, D.L. Adams ${ }^{24}$, T.N. Addy ${ }^{56}$, J. Adelman ${ }^{176}$, S. Adomeit ${ }^{98}$, P. Adragna ${ }^{75}$, T. Adye ${ }^{129}$, S. Aefsky ${ }^{22}$, J.A. Aguilar-Saavedra ${ }^{124 b, a}$, M. Agustoni ${ }^{16}$, M. Aharrouche ${ }^{81}$, S.P. Ahlen ${ }^{21}$, F. Ahles ${ }^{48}$, A. Ahmad ${ }^{148}$, M. Ahsan ${ }^{40}$, G. Aielli133a,133b, T. Akdogan ${ }^{18 a}$, T.P.A. Åkesson ${ }^{79}$, G. Akimoto ${ }^{155}$, A.V. Akimov ${ }^{94}$, M.S. Alam ${ }^{1}$, M.A. Alam ${ }^{76}$, J. Albert ${ }^{169}$, S. Albrand ${ }^{55}$, M. Aleksa ${ }^{29}$, I.N. Aleksandrov ${ }^{64}$, F. Alessandria ${ }^{89 a}$, C. Alexa ${ }^{25 a}$, G. Alexander ${ }^{153}$, G. Alexandre ${ }^{49}$, T. Alexopoulos ${ }^{9}$, M. Alhroob ${ }^{164 a, 164 c}$, M. Aliev $^{15}$, G. Alimonti ${ }^{89 a}$, J. Alison ${ }^{120}$, B.M.M. Allbrooke ${ }^{17}$, P.P. Allport ${ }^{73}$, S.E. Allwood-Spiers ${ }^{53}$, J. Almond ${ }^{82}$, A. Aloisio ${ }^{102 a, 102 b}$, R. Alon ${ }^{172}$, A. Alonso ${ }^{79}$, B. Alvarez Gonzalez ${ }^{88}$, M.G. Alviggi 102a,102b, K. Amako ${ }^{65}$, C. Amelung ${ }^{22}$, V.V. Ammosov ${ }^{128}$, A. Amorim ${ }^{124 a, b}$, N. Amram ${ }^{153}$, C. Anastopoulos ${ }^{29}$, L.S. Ancu ${ }^{16}$, N. Andari ${ }^{115}$, T. Andeen ${ }^{34}$, C.F. Anders ${ }^{58 b}$, G. Anders ${ }^{58 a}$, K.J. Anderson ${ }^{30}$, A. Andreazza ${ }^{89 a, 89 b}$, V. Andrei ${ }^{58 a}$, X.S. Anduaga ${ }^{70}$, P. Anger ${ }^{43}$, A. Angerami ${ }^{34}$, F. Anghinolfi ${ }^{29}$, A. Anisenkov ${ }^{107}$, N. Anjos ${ }^{124 a}$, A. Annovi ${ }^{47}$, A. Antonaki ${ }^{8}$, M. Antonelli ${ }^{47}$, A. Antonov ${ }^{96}$, J. Antos ${ }^{144 b}$, F. Anulli ${ }^{132 a}$, S. Aoun ${ }^{83}$, L. Aperio Bella ${ }^{4}$, R. Apolle ${ }^{118, c}$, G. Arabidze ${ }^{88}$, I. Aracena ${ }^{143}$, Y. Arai ${ }^{65}$, A.T.H. Arce ${ }^{44}$, S. Arfaoui ${ }^{148}$,

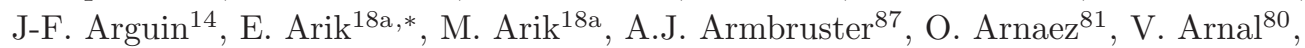
C. Arnault ${ }^{115}$, A. Artamonov ${ }^{95}$, G. Artoni ${ }^{132 a, 132 b}$, D. Arutinov ${ }^{20}$, S. Asai ${ }^{155}$, R. Asfandiyarov ${ }^{173}$, S. Ask ${ }^{27}$, B. Åsman 146a,146b, L. Asquith ${ }^{5}$, K. Assamagan ${ }^{24}$, A. Astbury ${ }^{169}$, B. Aubert ${ }^{4}$, E. Auge ${ }^{115}$, K. Augsten ${ }^{127}$, M. Aurousseau ${ }^{145 a}$, G. Avolio ${ }^{163}$, R. Avramidou ${ }^{9}$, D. Axen ${ }^{168}$, G. Azuelos ${ }^{93, d}$, Y. Azuma ${ }^{155}$, M.A. Baak ${ }^{29}$, G. Baccaglioni89a , C. Bacci'134a,134b, A.M. Bach ${ }^{14}$, H. Bachacou ${ }^{136}$, K. Bachas ${ }^{29}$, M. Backes ${ }^{49}$, M. Backhaus ${ }^{20}$, E. Badescu ${ }^{25 a}$, P. Bagnaia ${ }^{132 a, 132 b}$, S. Bahinipati ${ }^{2}$, Y. Bai ${ }^{32 a}$, D.C. Bailey ${ }^{158}$, T. Bain ${ }^{158}$, J.T. Baines ${ }^{129}$, O.K. Baker ${ }^{176}$, M.D. Baker ${ }^{24}$, S. Baker ${ }^{77}$, E. Banas ${ }^{38}$, P. Banerjee ${ }^{93}$, Sw. Banerjee ${ }^{173}$, D. Banfi ${ }^{29}$, A. Bangert ${ }^{150}$, V. Bansal ${ }^{169}$, H.S. Bansil ${ }^{17}$, L. Barak ${ }^{172}$, S.P. Baranov ${ }^{94}$, A. Barbaro Galtieri ${ }^{14}$, T. Barber ${ }^{48}$, E.L. Barberio ${ }^{86}$, D. Barberis ${ }^{50 a, 50 b}$, M. Barbero ${ }^{20}$, D.Y. Bardin ${ }^{64}$, T. Barillari ${ }^{99}$, M. Barisonzi ${ }^{175}$, T. Barklow ${ }^{143}$, N. Barlow ${ }^{27}$, B.M. Barnett ${ }^{129}$, R.M. Barnett ${ }^{14}$, A. Baroncelli ${ }^{134 a}$, G. Barone ${ }^{49}$, A.J. Barr ${ }^{118}$, F. Barreiro ${ }^{80}$, J. Barreiro Guimarães da Costa ${ }^{57}$, P. Barrillon ${ }^{115}$, R. Bartoldus ${ }^{143}$, A.E. Barton ${ }^{71}$, V. Bartsch ${ }^{149}$, R.L. Bates ${ }^{53}$, L. Batkova ${ }^{144 a}$, J.R. Batley ${ }^{27}$, A. Battaglia ${ }^{16}$, M. Battistin ${ }^{29}$, F. Bauer ${ }^{136}$, H.S. Bawa ${ }^{143, e}$, S. Beale ${ }^{98}$, T. Beau ${ }^{78}$, P.H. Beauchemin ${ }^{161}$, R. Beccherle ${ }^{50 a}$, P. Bechtle ${ }^{20}$, H.P. Beck ${ }^{16}$, A.K. Becker ${ }^{175}$, S. Becker ${ }^{98}$, M. Beckingham ${ }^{138}$, K.H. Becks ${ }^{175}$, A.J. Beddall ${ }^{18 c}$, A. Beddall ${ }^{18 c}$, S. Bedikian ${ }^{176}$, V.A. Bednyakov ${ }^{64}$, C.P. Bee ${ }^{83}$, M. Begel ${ }^{24}$, S. Behar Harpaz ${ }^{152}$, M. Beimforde ${ }^{99}$, C. Belanger-Champagne ${ }^{85}$, P.J. Bell ${ }^{49}$, W.H. Bell ${ }^{49}$, G. Bella ${ }^{153}$, L. Bellagamba ${ }^{19 a}$, F. Bellina $^{29}$, M. Bellomo $^{29}$, A. Belloni ${ }^{57}$, O. Beloborodova ${ }^{107, f}$, K. Belotskiy ${ }^{96}$, O. Beltramello ${ }^{29}$, O. Benary ${ }^{153}$, D. Benchekroun ${ }^{135 a}$, K. Bendtz ${ }^{146 a, 146 b}$, N. Benekos ${ }^{165}$, Y. Benhammou ${ }^{153}$, E. Benhar Noccioli ${ }^{49}$, J.A. Benitez Garcia ${ }^{159 b}$, D.P. Benjamin ${ }^{44}$, M. Benoit ${ }^{115}$, J.R. Bensinger ${ }^{22}$, K. Benslama ${ }^{130}$, S. Bentvelsen ${ }^{105}$, D. Berge ${ }^{29}$, E. Bergeaas Kuutmann ${ }^{41}$, N. Berger ${ }^{4}$, F. Berghaus ${ }^{169}$, E. Berglund ${ }^{105}$, J. Beringer ${ }^{14}$, P. Bernat ${ }^{77}$, R. Bernhard ${ }^{48}$, C. Bernius ${ }^{24}$, T. Berry ${ }^{76}$, C. Bertella ${ }^{83}$, A. Bertin ${ }^{19 a, 19 b}$, F. Bertolucci ${ }^{122 a, 122 b}$, M.I. Besana ${ }^{89 a, 89 b}$, G.J. Besjes ${ }^{104}$,

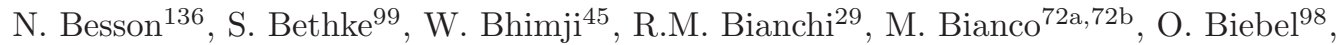
S.P. Bieniek ${ }^{77}$, K. Bierwagen ${ }^{54}$, J. Biesiada ${ }^{14}$, M. Biglietti134a , H. Bilokon ${ }^{47}$, M. Bindi ${ }^{19 a, 19 b}$, S. Binet ${ }^{115}$, A. Bingul ${ }^{18 c}$, C. Bini ${ }^{132 a, 132 b}$, C. Biscarat ${ }^{178}$, U. Bitenc ${ }^{48}$, K.M. Black ${ }^{21}$, R.E. Blair ${ }^{5}$, J.-B. Blanchard ${ }^{136}$, G. Blanchot ${ }^{29}$, T. Blazek ${ }^{144 a}$, C. Blocker ${ }^{22}$, J. Blocki ${ }^{38}$, A. Blondel ${ }^{49}$, W. Blum ${ }^{81}$, U. Blumenschein ${ }^{54}$, G.J. Bobbink ${ }^{105}$, V.B. Bobrovnikov ${ }^{107}$, S.S. Bocchetta ${ }^{79}$, A. Bocci ${ }^{44}$, C.R. Boddy ${ }^{118}$, M. Boehler ${ }^{41}$, J. Boek ${ }^{175}$, N. Boelaert ${ }^{35}$, J.A. Bogaerts ${ }^{29}$, 
A. Bogdanchikov ${ }^{107}$, A. Bogouch ${ }^{90, *}$, C. Bohm ${ }^{146 a}$, J. Bohm ${ }^{125}$, V. Boisvert ${ }^{76}$, T. Bold ${ }^{37}$, V. Boldea ${ }^{25 a}$, N.M. Bolnet ${ }^{136}$, M. Bomben ${ }^{78}$, M. Bona ${ }^{75}$, M. Boonekamp ${ }^{136}$, C.N. Booth ${ }^{139}$, S. Bordoni ${ }^{78}$, C. Borer ${ }^{16}$, A. Borisov ${ }^{128}$, G. Borissov ${ }^{71}$, I. Borjanovic ${ }^{12 a}$, M. Borri ${ }^{82}$, S. Borroni ${ }^{87}$, V. Bortolotto ${ }^{134 a, 134 b}$, K. Bos ${ }^{105}$, D. Boscherini ${ }^{19 a}$, M. Bosman $^{11}$, H. Boterenbrood ${ }^{105}$, D. Botterill ${ }^{129}$, J. Bouchami ${ }^{93}$, J. Boudreau ${ }^{123}$, E.V. Bouhova-Thacker ${ }^{71}$, D. Boumediene ${ }^{33}$, C. Bourdarios ${ }^{115}$, N. Bousson ${ }^{83}$, A. Boveia ${ }^{30}$, J. Boyd ${ }^{29}$, I.R. Boyko ${ }^{64}$, I. Bozovic-Jelisavcic ${ }^{12 b}$, J. Bracinik ${ }^{17}$, P. Branchini1 ${ }^{134 a}$, A. Brandt ${ }^{7}$, G. Brandt ${ }^{118}$, O. Brandt ${ }^{54}$, U. Bratzler ${ }^{156}$, B. Brau ${ }^{84}$, J.E. Brau ${ }^{114}$, H.M. Braun ${ }^{175}$, S.F. Brazzale ${ }^{164 a, 164 c}$, B. Brelier ${ }^{158}$, J. Bremer ${ }^{29}$, K. Brendlinger ${ }^{120}$, R. Brenner ${ }^{166}$, S. Bressler ${ }^{172}$, D. Britton ${ }^{53}$, F.M. Brochu ${ }^{27}$, I. Brock ${ }^{20}$, R. Brock ${ }^{88}$, E. Brodet ${ }^{153}$, F. Broggi ${ }^{89 a}$, C. Bromberg ${ }^{88}$, J. Bronner ${ }^{99}$, G. Brooijmans ${ }^{34}$, T. Brooks ${ }^{76}$, W.K. Brooks ${ }^{31 b}$, G. Brown ${ }^{82}$, H. Brown ${ }^{7}$, P.A. Bruckman de Renstrom ${ }^{38}$, D. Bruncko ${ }^{144 \mathrm{~b}}$, R. Bruneliere ${ }^{48}$, S. Brunet ${ }^{60}$, A. Bruni ${ }^{19 a}$, G. Bruni ${ }^{19 a}$, M. Bruschi ${ }^{19 a}$, T. Buanes ${ }^{13}$, Q. Buat ${ }^{55}$, F. Bucci ${ }^{49}$, J. Buchanan ${ }^{118}$, P. Buchholz ${ }^{141}$, R.M. Buckingham ${ }^{118}$, A.G. Buckley ${ }^{45}$, S.I. Buda ${ }^{25 a}$, I.A. Budagov ${ }^{64}$, B. Budick ${ }^{108}$, V. Büscher ${ }^{81}$, L. Bugge ${ }^{117}$, O. Bulekov ${ }^{96}$, A.C. Bundock ${ }^{73}$,

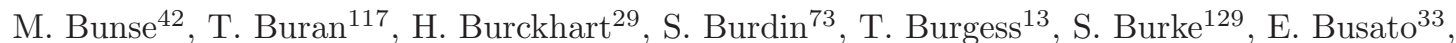
P. Bussey ${ }^{53}$, C.P. Buszello ${ }^{166}$, B. Butler ${ }^{143}$, J.M. Butler ${ }^{21}$, C.M. Buttar ${ }^{53}$, J.M. Butterworth ${ }^{77}$, W. Buttinger ${ }^{27}$, S. Cabrera Urbán ${ }^{167}$, D. Caforio ${ }^{19 a, 19 b}$, O. Cakir ${ }^{3 a}$, P. Calafiura ${ }^{14}$, G. Calderini ${ }^{78}$, P. Calfayan ${ }^{98}$, R. Calkins ${ }^{106}$, L.P. Caloba ${ }^{23 a}$, R. Caloi ${ }^{132 a, 132 b}$, D. Calvet $^{33}$, S. Calvet ${ }^{33}$, R. Camacho Toro ${ }^{33}$, P. Camarri133a,133b, D. Cameron ${ }^{117}$, L.M. Caminada ${ }^{14}$, S. Campana ${ }^{29}$, M. Campanelli ${ }^{77}$, V. Canale ${ }^{102 a, 102 b}$, F. Canelli $^{30, g}$, A. Canepa ${ }^{159 a}$, J. Cantero ${ }^{80}$, R. Cantrill ${ }^{76}$, L. Capasso ${ }^{102 a, 102 b}$, M.D.M. Capeans Garrido ${ }^{29}$, I. Caprini ${ }^{25 a}$, M. Caprini ${ }^{25 a}$, D. Capriotti ${ }^{99}$, M. Capua ${ }^{36 a, 36 b}$, R. Caputo ${ }^{81}$, R. Cardarelli ${ }^{133 a}$, T. Carli ${ }^{29}$, G. Carlino ${ }^{102 a}$, L. Carminati ${ }^{89 a, 89 b}$, B. Caron $^{85}$, S. Caron ${ }^{104}$, E. Carquin ${ }^{31 b}$, G.D. Carrillo Montoya ${ }^{173}$, A.A. Carter $^{75}$, J.R. Carter ${ }^{27}$, J. Carvalho ${ }^{124 a, h}$, D. Casadei ${ }^{108}$, M.P. Casado ${ }^{11}$,

M. Cascella ${ }^{122 a, 122 b}$, C. Caso ${ }^{50 a, 50 b, *}$, A.M. Castaneda Hernandez ${ }^{173, i}$, E. Castaneda-Miranda ${ }^{173}$, V. Castillo Gimenez ${ }^{167}$, N.F. Castro ${ }^{124 a}$, G. Cataldi ${ }^{72 a}$, P. Catastini ${ }^{57}$, A. Catinaccio ${ }^{29}$, J.R. Catmore ${ }^{29}$, A. Cattai ${ }^{29}$, G. Cattani ${ }^{133 a, 133 b}$, S. Caughron ${ }^{88}$, P. Cavalleri $^{78}$, D. Cavalli ${ }^{89 a}$, M. Cavalli-Sforza ${ }^{11}$, V. Cavasinni ${ }^{122 a, 122 b}$, F. Ceradini ${ }^{134 a, 134 b}$, A.S. Cerqueira ${ }^{23 b}$, A. Cerri ${ }^{29}$, L. Cerrito ${ }^{75}$, F. Cerutti ${ }^{47}$, S.A. Cetin ${ }^{18 b}$, A. Chafaq ${ }^{135 a}$, D. Chakraborty ${ }^{106}$, I. Chalupkova ${ }^{126}$, K. Chan $^{2}$, B. Chapleau ${ }^{85}$, J.D. Chapman ${ }^{27}$, J.W. Chapman ${ }^{87}$, E. Chareyre ${ }^{78}$, D.G. Charlton ${ }^{17}$, V. Chavda ${ }^{82}$, C.A. Chavez Barajas ${ }^{29}$, S. Cheatham ${ }^{85}$, S. Chekanov ${ }^{5}$, S.V. Chekulaev ${ }^{159 a}$, G.A. Chelkov ${ }^{64}$, M.A. Chelstowska ${ }^{104}$, C. Chen ${ }^{63}$, H. Chen ${ }^{24}$, S. Chen ${ }^{32 c}$, X. Chen ${ }^{173}$, Y. Chen ${ }^{34}$, A. Cheplakov ${ }^{64}$, R. Cherkaoui El Moursli ${ }^{135 e}$, V. Chernyatin ${ }^{24}$, E. Cheu ${ }^{6}$, S.L. Cheung ${ }^{158}$, L. Chevalier ${ }^{136}$, G. Chiefari102a,102b, L. Chikovani ${ }^{51 a}$, J.T. Childers ${ }^{29}$, A. Chilingarov ${ }^{71}$, G. Chiodini ${ }^{72 a}$, A.S. Chisholm ${ }^{17}$, R.T. Chislett ${ }^{77}$, A. Chitan ${ }^{25 a}$, M.V. Chizhov ${ }^{64}$, G. Choudalakis $^{30}$, S. Chouridou ${ }^{137}$, I.A. Christidi ${ }^{77}$, A. Christov ${ }^{48}$, D. Chromek-Burckhart ${ }^{29}$, M.L. Chu ${ }^{151}$, J. Chudoba ${ }^{125}$, G. Ciapetti ${ }^{132 a, 132 b}$, A.K. Ciftci ${ }^{3 a}$, R. Ciftci ${ }^{3 a}$, D. Cinca ${ }^{33}$, V. Cindro ${ }^{74}$, C. Ciocca ${ }^{19 a, 19 b}$, A. Ciocio $^{14}$, M. Cirilli ${ }^{87}$, P. Cirkovic ${ }^{12 b}$, M. Citterio ${ }^{89 a}$, M. Ciubancan ${ }^{25 a}$, A. Clark ${ }^{49}$, P.J. Clark ${ }^{45}$, R.N. Clarke ${ }^{14}$, W. Cleland ${ }^{123}$, J.C. Clemens ${ }^{83}$, B. Clement ${ }^{55}$, C. Clement ${ }^{146 a, 146 b}$, Y. Coadou ${ }^{83}$, M. Cobal ${ }^{164 a, 164 c}$, A. Coccaro ${ }^{138}$, J. Cochran ${ }^{63}$, J.G. Cogan ${ }^{143}$, J. Coggeshall ${ }^{165}$, E. Cogneras ${ }^{178}$, J. Colas ${ }^{4}$, A.P. Colijn ${ }^{105}$, N.J. Collins ${ }^{17}$, C. Collins-Tooth ${ }^{53}$, J. Collot ${ }^{55}$, T. Colombo ${ }^{119 a, 119 b}$, G. Colon ${ }^{84}$, P. Conde Muiño ${ }^{124 a}$, E. Coniavitis ${ }^{118}$, M.C. Conidi ${ }^{11}$, S.M. Consonni ${ }^{89 a, 89 b}$, V. Consorti ${ }^{48}$, S. Constantinescu ${ }^{25 a}$, C. Conta ${ }^{119 a, 119 b}$, G. Conti ${ }^{57}$, F. Conventi1 ${ }^{102 a, j}$, M. Cooke ${ }^{14}$, B.D. Cooper ${ }^{77}$, A.M. Cooper-Sarkar ${ }^{118}$, K. Copic ${ }^{14}$, T. Cornelissen ${ }^{175}$, M. Corradi ${ }^{19 a}$, F. Corriveau ${ }^{85, k}$,

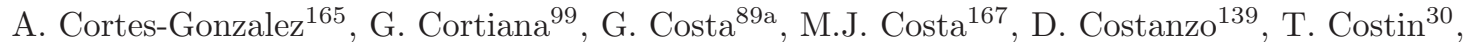
D. Côté ${ }^{29}$, L. Courneyea ${ }^{169}$, G. Cowan ${ }^{76}$, C. Cowden ${ }^{27}$, B.E. Cox ${ }^{82}$, K. Cranmer ${ }^{108}$, F. Crescioli ${ }^{122 a, 122 b}$, M. Cristinziani ${ }^{20}$, G. Crosetti ${ }^{36 a, 36 b}$, R. Crupi ${ }^{72 a, 72 b}$, S. Crépé-Renaudin ${ }^{55}$, 
C.-M. Cuciuc ${ }^{25 a}$, C. Cuenca Almenar ${ }^{176}$, T. Cuhadar Donszelmann ${ }^{139}$, M. Curatolo ${ }^{47}$, C.J. Curtis ${ }^{17}$, C. Cuthbert ${ }^{150}$, P. Cwetanski ${ }^{60}$, H. Czirr ${ }^{141}$, P. Czodrowski ${ }^{43}$, Z. Czyczula ${ }^{176}$, S. D’Auria ${ }^{53}$, M. D'Onofrio ${ }^{73}$, A. D’Orazio ${ }^{132 a, 132 b}$, M.J. Da Cunha Sargedas De Sousa ${ }^{124 a}$, C. Da Via ${ }^{82}$, W. Dabrowski ${ }^{37}$, A. Dafinca ${ }^{118}$, T. Dai ${ }^{87}$, C. Dallapiccola ${ }^{84}$, M. Dam ${ }^{35}$, M. Dameri ${ }^{50 a, 50 b}$, D.S. Damiani ${ }^{137}$, H.O. Danielsson ${ }^{29}$, V. Dao ${ }^{49}$, G. Darbo ${ }^{50 a}$, G.L. Darlea ${ }^{25 b}$, W. Davey ${ }^{20}$, T. Davidek ${ }^{126}$, N. Davidson ${ }^{86}$, R. Davidson ${ }^{71}$, E. Davies ${ }^{118, c}$, M. Davies ${ }^{93}$, A.R. Davison ${ }^{77}$, Y. Davygora ${ }^{58 a}$, E. Dawe ${ }^{142}$, I. Dawson ${ }^{139}$, R.K. Daya-Ishmukhametova ${ }^{22}$,

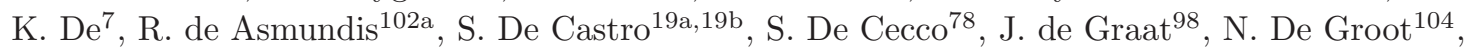
P. de Jong ${ }^{105}$, C. De La Taille ${ }^{115}$, H. De la Torre ${ }^{80}$, F. De Lorenzi ${ }^{63}$, L. de Mora ${ }^{71}$, L. De Nooij ${ }^{105}$, D. De Pedis ${ }^{132 a}$, A. De Salvo ${ }^{132 a}$, U. De Sanctis ${ }^{164 a, 164 c}$, A. De Santo ${ }^{149}$, J.B. De Vivie De Regie ${ }^{115}$, G. De Zorzi ${ }^{132 a, 132 b}$, W.J. Dearnaley ${ }^{71}$, R. Debbe ${ }^{24}$, C. Debenedetti ${ }^{45}$, B. Dechenaux ${ }^{55}$, D.V. Dedovich ${ }^{64}$, J. Degenhardt ${ }^{120}$, C. Del Papa ${ }^{164 a, 164 c}$, J. Del Peso ${ }^{80}$, T. Del Prete ${ }^{122 a, 122 b}$, T. Delemontex ${ }^{55}$, M. Deliyergiyev ${ }^{74}$, A. Dell'Acqua ${ }^{29}$, L. Dell'Asta ${ }^{21}$, M. Della Pietra ${ }^{102 a, j}$, D. della Volpe ${ }^{102 a, 102 b}$, M. Delmastro ${ }^{4}$, P.A. Delsart ${ }^{55}$, C. Deluca ${ }^{105}$, S. Demers ${ }^{176}$, M. Demichev ${ }^{64}$, B. Demirkoz ${ }^{11, l}$, J. Deng ${ }^{163}$, S.P. Denisov ${ }^{128}$, D. Derendarz ${ }^{38}$,

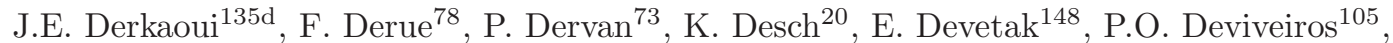
A. Dewhurst ${ }^{129}$, B. DeWilde ${ }^{148}$, S. Dhaliwal ${ }^{158}$, R. Dhullipudi ${ }^{24, m}$, A. Di Ciaccio ${ }^{133 a, 133 b}$, L. Di Ciaccio ${ }^{4}$, A. Di Girolamo ${ }^{29}$, B. Di Girolamo ${ }^{29}$, S. Di Luise ${ }^{134 a, 134 b}$, A. Di Mattia ${ }^{173}$, B. Di Micco ${ }^{29}$, R. Di Nardo ${ }^{47}$, A. Di Simone ${ }^{133 a, 133 b}$, R. Di Sipio ${ }^{19 a, 19 b}$, M.A. Diaz ${ }^{31 a}$, E.B. Diehl ${ }^{87}$, J. Dietrich ${ }^{41}$, T.A. Dietzsch ${ }^{58 a}$, S. Diglio ${ }^{86}$, K. Dindar Yagci $^{39}$, J. Dingfelder ${ }^{20}$,

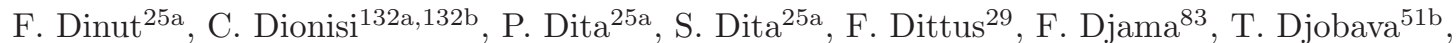
M.A.B. do Vale ${ }^{23 c}$, A. Do Valle Wemans ${ }^{124 a, n}$, T.K.O. Doan ${ }^{4}$, M. Dobbs ${ }^{85}$, R. Dobinson ${ }^{29, *}$, D. $\operatorname{Dobos}^{29}$, E. Dobson ${ }^{29, o}$, J. Dodd ${ }^{34}$, C. Doglioni ${ }^{49}$, T. Doherty ${ }^{53}$, Y. Doi ${ }^{65, *}$, J. Dolejsi ${ }^{126}$, I. Dolenc ${ }^{74}$, Z. Dolezal ${ }^{126}$, B.A. Dolgoshein ${ }^{96, *}$, T. Dohmae ${ }^{155}$, M. Donadelli ${ }^{23 d}$, J. Donini ${ }^{33}$, J. Dopke ${ }^{29}$, A. Doria ${ }^{102 a}$, A. Dos Anjos ${ }^{173}$, A. Dotti ${ }^{122 a, 122 b}$, M.T. Dova ${ }^{70}$, A.D. Doxiadis ${ }^{105}$, A.T. Doyle ${ }^{53}$, M. Dris ${ }^{9}$, J. Dubbert ${ }^{99}$, S. Dube ${ }^{14}$, E. Duchovni ${ }^{172}$, G. Duckeck ${ }^{98}$, A. Dudarev ${ }^{29}$, F. Dudziak ${ }^{63}$, M. Dührssen ${ }^{29}$, I.P. Duerdoth ${ }^{82}$, L. Duflot ${ }^{115}$, M-A. Dufour ${ }^{85}$, M. Dunford ${ }^{29}$, H. Duran Yildiz ${ }^{3 a}$, R. Duxfield ${ }^{139}$, M. Dwuznik ${ }^{37}$, F. Dydak ${ }^{29}$, M. Düren ${ }^{52}$, J. Ebke ${ }^{98}$, S. Eckweiler ${ }^{81}$, K. Edmonds ${ }^{81}$, C.A. Edwards ${ }^{76}$, N.C. Edwards ${ }^{53}$, W. Ehrenfeld ${ }^{41}$, T. Eifert ${ }^{143}$, G. Eigen ${ }^{13}$, K. Einsweiler ${ }^{14}$, E. Eisenhandler ${ }^{75}$, T. Ekelof ${ }^{166}$, M. El Kacimi ${ }^{135 c}$, M. Ellert ${ }^{166}$, S. Elles ${ }^{4}$, F. Ellinghaus ${ }^{81}$, K. Ellis ${ }^{75}$, N. Ellis ${ }^{29}$, J. Elmsheuser ${ }^{98}$, M. Elsing ${ }^{29}$, D. Emeliyanov ${ }^{129}$, R. Engelmann ${ }^{148}$, A. Engl ${ }^{98}$, B. Epp ${ }^{61}$, A. Eppig ${ }^{87}$, J. Erdmann ${ }^{54}$, A. Ereditato ${ }^{16}$, D. Eriksson ${ }^{146 a}$, J. Ernst ${ }^{1}$, M. Ernst ${ }^{24}$, J. Ernwein ${ }^{136}$, D. Errede ${ }^{165}$, S. Errede ${ }^{165}$, E. Ertel ${ }^{81}$, M. Escalier ${ }^{115}$, H. Esch ${ }^{42}$, C. Escobar ${ }^{123}$, X. Espinal Curull ${ }^{11}$, B. Esposito ${ }^{47}$, F. Etienne ${ }^{83}$, A.I. Etienvre ${ }^{136}$, E. Etzion ${ }^{153}$, D. Evangelakou ${ }^{54}$, H. Evans ${ }^{60}$, L. Fabbri ${ }^{19 a, 19 b}$, C. Fabre ${ }^{29}$, R.M. Fakhrutdinov ${ }^{128}$, S. Falciano ${ }^{132 a}$, Y. Fang ${ }^{173}$, M. Fanti ${ }^{89 a, 89 b}$, A. Farbin ${ }^{7}$, A. Farilla ${ }^{134 a}$, J. Farley ${ }^{148}$, T. Farooque ${ }^{158}$, S. Farrell ${ }^{163}$, S.M. Farrington ${ }^{118}$, P. Farthouat ${ }^{29}$, P. Fassnacht ${ }^{29}$, D. Fassouliotis ${ }^{8}$, B. Fatholahzadeh ${ }^{158}$, A. Favareto ${ }^{89 a, 89 b}$, L. Fayard ${ }^{115}$, S. Fazio ${ }^{36 a, 36 b}$, R. Febbraro ${ }^{33}$, P. Federic ${ }^{144 a}$, O.L. Fedin ${ }^{121}$, W. Fedorko ${ }^{88}$, M. Fehling-Kaschek ${ }^{48}$, L. Feligioni ${ }^{83}$, D. Fellmann ${ }^{5}$, C. Feng ${ }^{32 d}$, E.J. Feng ${ }^{5}$, A.B. Fenyuk ${ }^{128}$, J. Ferencei ${ }^{144 b}$, W. Fernando ${ }^{5}$, S. Ferrag $^{53}$, J. Ferrando ${ }^{53}$, V. Ferrara ${ }^{41}$, A. Ferrari ${ }^{166}$, P. Ferrari ${ }^{105}$, R. Ferrari ${ }^{119 a}$, D.E. Ferreira de Lima ${ }^{53}$, A. Ferrer ${ }^{167}$, D. Ferrere ${ }^{49}$, C. Ferretti ${ }^{87}$, A. Ferretto Parodi ${ }^{50 a, 50 b}$, M. Fiascaris ${ }^{30}$, F. Fiedler ${ }^{81}$, A. Filipčič ${ }^{74}$, F. Filthaut ${ }^{104}$, M. Fincke-Keeler ${ }^{169}$, M.C.N. Fiolhais ${ }^{124 a, h}$, L. Fiorini ${ }^{167}$, A. Firan ${ }^{39}$, G. Fischer ${ }^{41}$, M.J. Fisher ${ }^{109}$, M. Flechl ${ }^{48}$, I. Fleck ${ }^{141}$, J. Fleckner ${ }^{81}$, P. Fleischmann ${ }^{174}$,

S. Fleischmann ${ }^{175}$, T. Flick ${ }^{175}$, A. Floderus ${ }^{79}$, L.R. Flores Castillo $^{173}$, M.J. Flowerdew ${ }^{99}$, T. Fonseca Martin ${ }^{16}$, A. Formica ${ }^{136}$, A. Forti ${ }^{82}$, D. Fortin ${ }^{159 a}$, D. Fournier ${ }^{115}$, H. Fox ${ }^{71}$, P. Francavilla ${ }^{11}$, S. Franchino ${ }^{119 a, 119 b}$, D. Francis ${ }^{29}$, T. Frank ${ }^{172}$, S. Franz ${ }^{29}$, M. Fraternali ${ }^{119 a, 119 b}$, S. Fratina ${ }^{120}$, S.T. French ${ }^{27}$, C. Friedrich ${ }^{41}$, F. Friedrich ${ }^{43}$, R. Froeschl ${ }^{29}$, 
D. Froidevaux ${ }^{29}$, J.A. Frost ${ }^{27}$, C. Fukunaga ${ }^{156}$, E. Fullana Torregrosa ${ }^{29}$, B.G. Fulsom ${ }^{143}$, J. Fuster ${ }^{167}$, C. Gabaldon ${ }^{29}$, O. Gabizon ${ }^{172}$, T. Gadfort ${ }^{24}$, S. Gadomski ${ }^{49}$, G. Gagliardi ${ }^{50 a, 50 b}$, P. Gagnon ${ }^{60}$, C. Galea ${ }^{98}$, E.J. Gallas ${ }^{118}$, V. Gallo ${ }^{16}$, B.J. Gallop ${ }^{129}$, P. Gallus ${ }^{125}$, K.K. Gan ${ }^{109}$, Y.S. Gao ${ }^{143, e}$, A. Gaponenko ${ }^{14}$, F. Garberson ${ }^{176}$, M. Garcia-Sciveres ${ }^{14}$, C. García ${ }^{167}$, J.E. García Navarro ${ }^{167}$, R.W. Gardner ${ }^{30}$, N. Garelli ${ }^{29}$, H. Garitaonandia ${ }^{105}$, V. Garonne ${ }^{29}$, J. Garvey ${ }^{17}$, C. Gatti ${ }^{47}$, G. Gaudio ${ }^{119 a}$, B. Gaur ${ }^{141}$, L. Gauthier ${ }^{136}$, P. Gauzzi ${ }^{132 a, 132 b}$, I.L. Gavrilenko ${ }^{94}$, C. Gay ${ }^{168}$, G. Gaycken ${ }^{20}$, E.N. Gazis ${ }^{9}$, P. Ge ${ }^{32 d}$, Z. Gecse ${ }^{168}$, C.N.P. Gee ${ }^{129}$, D.A.A. Geerts ${ }^{105}$, Ch. Geich-Gimbel ${ }^{20}$, K. Gellerstedt ${ }^{146 a, 146 b}$, C. Gemme ${ }^{50 a}$, A. Gemmell ${ }^{53}$, M.H. Genest ${ }^{55}$, S. Gentile ${ }^{132 a, 132 b}$, M. George ${ }^{54}$, S. George ${ }^{76}$, P. Gerlach ${ }^{175}$, A. Gershon ${ }^{153}$, C. Geweniger ${ }^{58 a}$, H. Ghazlane ${ }^{135 b}$, N. Ghodbane ${ }^{33}$, B. Giacobbe ${ }^{19 a}$, S. Giagu ${ }^{132 a, 132 b}$, V. Giakoumopoulou ${ }^{8}$, V. Giangiobbe ${ }^{11}$, F. Gianotti ${ }^{29}$, B. Gibbard ${ }^{24}$, A. Gibson ${ }^{158}$, S.M. Gibson ${ }^{29}$, D. Gillberg ${ }^{28}$, A.R. Gillman ${ }^{129}$, D.M. Gingrich ${ }^{2, d}$, J. Ginzburg ${ }^{153}$, N. Giokaris ${ }^{8}$, M.P. Giordani ${ }^{164 c}$, R. Giordano ${ }^{102 a}$,102b , F.M. Giorgi ${ }^{15}$, P. Giovannini ${ }^{99}$, P.F. Giraud ${ }^{136}$, D. Giugni ${ }^{89 a}$, M. Giunta ${ }^{93}$, P. Giusti ${ }^{19 a}$, B.K. Gjelsten ${ }^{117}$, L.K. Gladilin ${ }^{97}$, C. Glasman ${ }^{80}$, J. Glatzer ${ }^{48}$, A. Glazov ${ }^{41}$, K.W. Glitza ${ }^{175}$, G.L. Glonti ${ }^{64}$, J.R. Goddard ${ }^{75}$, J. Godfrey ${ }^{142}$, J. Godlewski ${ }^{29}$, M. Goebel ${ }^{41}$, T. Göpfert ${ }^{43}$, C. Goeringer ${ }^{81}$, C. Gössling ${ }^{42}$, S. Goldfarb ${ }^{87}$, T. Golling ${ }^{176}$, A. Gomes ${ }^{124 a, b}$, L.S. Gomez Fajardo ${ }^{41}$, R. Gonçalo ${ }^{76}$, J. Goncalves Pinto Firmino Da Costa ${ }^{41}$, L. Gonella ${ }^{20}$, S. Gonzalez ${ }^{173}$, S. González de la $\mathrm{Hoz}^{167}$, G. Gonzalez Parra ${ }^{11}$, M.L. Gonzalez Silva ${ }^{26}$, S. Gonzalez-Sevilla ${ }^{49}$, J.J. Goodson ${ }^{148}$, L. Goossens ${ }^{29}$, P.A. Gorbounov ${ }^{95}$, H.A. Gordon ${ }^{24}$, I. Gorelov ${ }^{103}$, G. Gorfine ${ }^{175}$, B. Gorini ${ }^{29}$, E. Gorini ${ }^{72 a, 72 b}$, A. Gorišek ${ }^{74}$, E. Gornicki ${ }^{38}$, B. Gosdzik ${ }^{41}$, A.T. Goshaw ${ }^{5}$, M. Gosselink ${ }^{105}$, M.I. Gostkin ${ }^{64}$, I. Gough Eschrich ${ }^{163}$, M. Gouighri135a, D. Goujdami135c , M.P. Goulette ${ }^{49}$, A.G. Goussiou ${ }^{138}$, C. Goy ${ }^{4}$, S. Gozpinar ${ }^{22}$, I. Grabowska-Bold ${ }^{37}$, P. Grafström ${ }^{19 a, 19 b}$, K-J. Grahn ${ }^{41}$, F. Grancagnolo ${ }^{72 a}$, S. Grancagnolo ${ }^{15}$, V. Grassi ${ }^{148}$, V. Gratchev ${ }^{121}$, N. Grau ${ }^{34}$, H.M. Gray ${ }^{29}$, J.A. Gray ${ }^{148}$, E. Graziani ${ }^{134 a}$, O.G. Grebenyuk ${ }^{121}$, T. Greenshaw ${ }^{73}$, Z.D. Greenwood ${ }^{24, m}$, K. Gregersen ${ }^{35}$, I.M. Gregor ${ }^{41}$, P. Grenier ${ }^{143}$, J. Griffiths ${ }^{138}$, N. Grigalashvili ${ }^{64}$, A.A. Grillo ${ }^{137}$, S. Grinstein ${ }^{11}$, Y.V. Grishkevich ${ }^{97}$, J.-F. Grivaz ${ }^{115}$, E. Gross ${ }^{172}$, J. Grosse-Knetter ${ }^{54}$, J. Groth-Jensen ${ }^{172}$, K. Grybel ${ }^{141}$, D. Guest ${ }^{176}$, C. Guicheney ${ }^{33}$, A. Guida ${ }^{72 a, 72 b}$, S. Guindon ${ }^{54}$, U. Gul ${ }^{53}$, H. Guler ${ }^{85, p}$, J. Gunther ${ }^{125}$, B. Guo ${ }^{158}$, J. Guo ${ }^{34}$, P. Gutierrez ${ }^{111}$, N. Guttman ${ }^{153}$, O. Gutzwiller ${ }^{173}$, C. Guyot ${ }^{136}$, C. Gwenlan ${ }^{118}$, C.B. Gwilliam ${ }^{73}$, A. Haas ${ }^{143}$, S. $\mathrm{Haas}^{29}$, C. Haber ${ }^{14}$, H.K. Hadavand ${ }^{39}$, D.R. Hadley ${ }^{17}$, P. Haefner ${ }^{20}$, F. Hahn ${ }^{29}$, S. Haider ${ }^{29}$, Z. Hajduk ${ }^{38}$, H. Hakobyan ${ }^{177}$, D. Hall ${ }^{118}$, J. Haller ${ }^{54}$,

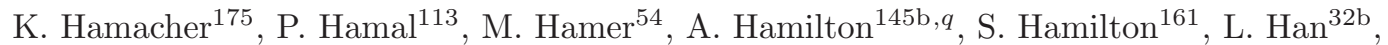
K. Hanagaki ${ }^{116}$, K. Hanawa ${ }^{160}$, M. Hance ${ }^{14}$, C. Handel $^{81}$, P. Hanke ${ }^{58 a}$, J.R. Hansen ${ }^{35}$,

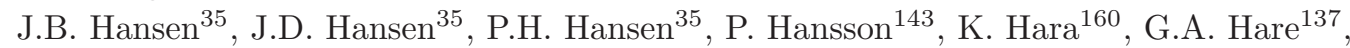
T. Harenberg ${ }^{175}$, S. Harkusha ${ }^{90}$, D. Harper ${ }^{87}$, R.D. Harrington ${ }^{45}$, O.M. Harris ${ }^{138}$, J. Hartert ${ }^{48}$, F. Hartjes ${ }^{105}$, T. Haruyama ${ }^{65}$, A. Harvey ${ }^{56}$, S. Hasegawa ${ }^{101}$, Y. Hasegawa ${ }^{140}$, S. Hassani ${ }^{136}$, S. Haug ${ }^{16}$, M. Hauschild ${ }^{29}$, R. Hauser ${ }^{88}$, M. Havranek ${ }^{20}$, C.M. Hawkes ${ }^{17}$, R.J. Hawkings ${ }^{29}$, A.D. Hawkins ${ }^{79}$, D. Hawkins ${ }^{163}$, T. Hayakawa ${ }^{66}$, T. Hayashi ${ }^{160}$, D. Hayden ${ }^{76}$, C.P. Hays ${ }^{118}$, H.S. Hayward ${ }^{73}$, S.J. Haywood ${ }^{129}$, M. He ${ }^{32 d}$, S.J. Head ${ }^{17}$, V. Hedberg ${ }^{79}$, L. Heelan ${ }^{7}$, S. Heim $^{88}$, B. Heinemann ${ }^{14}$, S. Heisterkamp ${ }^{35}$, L. Helary ${ }^{21}$, C. Heller ${ }^{98}$, M. Heller ${ }^{29}$, S. Hellman 146a,146b, D. Hellmich ${ }^{20}$, C. Helsens ${ }^{11}$, R.C.W. Henderson ${ }^{71}$, M. Henke ${ }^{58 \mathrm{a}}$, A. Henrichs ${ }^{54}$, A.M. Henriques Correia ${ }^{29}$, S. Henrot-Versille ${ }^{115}$, C. Hensel ${ }^{54}$, T. Henß ${ }^{175}$, C.M. Hernandez ${ }^{7}$, Y. Hernández Jiménez ${ }^{167}$, R. Herrberg ${ }^{15}$, G. Herten ${ }^{48}$, R. Hertenberger ${ }^{98}$, L. Hervas ${ }^{29}$, G.G. Hesketh ${ }^{77}$, N.P. Hessey ${ }^{105}$, E. Higón-Rodriguez ${ }^{167}$, J.C. Hill ${ }^{27}$, K.H. Hiller ${ }^{41}$, S. Hillert ${ }^{20}$, S.J. Hillier ${ }^{17}$, I. Hinchliffe ${ }^{14}$, E. Hines ${ }^{120}$, M. Hirose ${ }^{116}$, F. Hirsch ${ }^{42}$, D. Hirschbuehl ${ }^{175}$, J. Hobbs ${ }^{148}$, N. Hod ${ }^{153}$, M.C. Hodgkinson ${ }^{139}$, P. Hodgson ${ }^{139}$, A. Hoecker ${ }^{29}$, M.R. Hoeferkamp ${ }^{103}$, J. Hoffman ${ }^{39}$, D. Hoffmann ${ }^{83}$, M. Hohlfeld ${ }^{81}$, M. Holder ${ }^{141}$, S.O. Holmgren ${ }^{146 a}$, T. Holy ${ }^{127}$, J.L. Holzbauer ${ }^{88}$, T.M. Hong ${ }^{120}$, L. Hooft van Huysduynen ${ }^{108}$, C. Horn ${ }^{143}$, S. Horner ${ }^{48}$, 


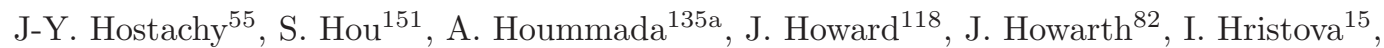
J. Hrivnac ${ }^{115}$, T. Hryn'ova ${ }^{4}$, P.J. Hsu ${ }^{81}$, S.-C. Hsu ${ }^{14}$, Z. Hubacek ${ }^{127}$, F. Hubaut ${ }^{83}$, F. Huegging ${ }^{20}$, A. Huettmann ${ }^{41}$, T.B. Huffman ${ }^{118}$, E.W. Hughes ${ }^{34}$, G. Hughes ${ }^{71}$, M. Huhtinen ${ }^{29}$, M. Hurwitz ${ }^{14}$, U. Husemann ${ }^{41}$, N. Huseynov ${ }^{64, r}$, J. Huston ${ }^{88}$, J. Huth ${ }^{57}$, G. Iacobucci ${ }^{49}$, G. Iakovidis ${ }^{9}$, M. Ibbotson ${ }^{82}$, I. Ibragimov ${ }^{141}$, L. Iconomidou-Fayard ${ }^{115}$, J. Idarraga ${ }^{115}$, P. Iengo ${ }^{102 a}$,

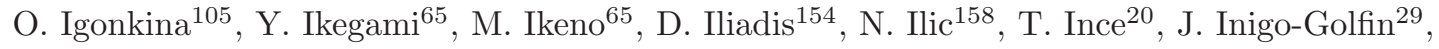
P. Ioannou ${ }^{8}$, M. Iodice ${ }^{134 a}$, K. Iordanidou ${ }^{8}$, V. Ippolito ${ }^{132 a, 132 b}$, A. Irles Quiles ${ }^{167}$, C. Isaksson ${ }^{166}$, M. Ishino ${ }^{67}$, M. Ishitsuka ${ }^{157}$, R. Ishmukhametov ${ }^{39}$, C. Issever ${ }^{118}$, S. Istin ${ }^{18 a}$, A.V. Ivashin ${ }^{128}$, W. Iwanski ${ }^{38}$, H. Iwasaki ${ }^{65}$, J.M. Izen ${ }^{40}$, V. Izzo ${ }^{102 a}$, B. Jackson ${ }^{120}$, J.N. Jackson ${ }^{73}$, P. Jackson ${ }^{143}$, M.R. Jaekel ${ }^{29}$, V. Jain ${ }^{60}$, K. Jakobs ${ }^{48}$, S. Jakobsen ${ }^{35}$, T. Jakoubek ${ }^{125}$, J. Jakubek ${ }^{127}$,

D.K. Jana ${ }^{111}$, E. Jansen ${ }^{77}$, H. Jansen ${ }^{29}$, A. Jantsch ${ }^{99}$, M. Janus ${ }^{48}$, G. Jarlskog ${ }^{79}$, L. Jeanty ${ }^{57}$, I. Jen-La Plante ${ }^{30}$, P. Jenni ${ }^{29}$, A. Jeremie ${ }^{4}$, P. Jež ${ }^{35}$, S. Jézéquel ${ }^{4}$, M.K. Jha ${ }^{19 a}$, H. Ji ${ }^{173}$, W. Ji ${ }^{81}$, J. Jia ${ }^{148}$, Y. Jiang ${ }^{32 b}$, M. Jimenez Belenguer ${ }^{41}$, S. Jin ${ }^{32 a}$, O. Jinnouchi ${ }^{157}$, M.D. Joergensen ${ }^{35}$, D. Joffe ${ }^{39}$, M. Johansen ${ }^{146 a, 146 b}$, K.E. Johansson ${ }^{146 a}$, P. Johansson ${ }^{139}$, S. Johnert ${ }^{41}$, K.A. Johns ${ }^{6}$, K. Jon-And ${ }^{146 a, 146 b}$, G. Jones ${ }^{170}$, R.W.L. Jones ${ }^{71}$, T.J. Jones ${ }^{73}$, C. Joram ${ }^{29}$, P.M. Jorge ${ }^{124 a}$, K.D. Joshi ${ }^{82}$, J. Jovicevic ${ }^{147}$, T. Jovin ${ }^{12 b}$, X. Ju ${ }^{173}$, C.A. Jung ${ }^{42}$, R.M. Jungst ${ }^{29}$, V. Juranek ${ }^{125}$, P. Jussel ${ }^{61}$, A. Juste Rozas ${ }^{11}$, S. Kabana ${ }^{16}$, M. Kaci ${ }^{167}$, A. Kaczmarska ${ }^{38}$, P. Kadlecik ${ }^{35}$, M. Kado ${ }^{115}$, H. Kagan ${ }^{109}$, M. Kagan ${ }^{57}$, E. Kajomovitz ${ }^{152}$, S. Kalinin ${ }^{175}$, L.V. Kalinovskaya ${ }^{64}$, S. Kama ${ }^{39}$, N. Kanaya ${ }^{155}$, M. Kaneda ${ }^{29}$, S. Kaneti27 ${ }^{27}$ T. Kanno ${ }^{157}$, V.A. Kantserov ${ }^{96}$, J. Kanzaki ${ }^{65}$, B. Kaplan ${ }^{176}$, A. Kapliy ${ }^{30}$, J. Kaplon ${ }^{29}$, D. Kar ${ }^{53}$, M. Karagounis ${ }^{20}$, K. Karakostas ${ }^{9}$, M. Karnevskiy ${ }^{41}$, V. Kartvelishvili ${ }^{71}$, A.N. Karyukhin ${ }^{128}$, L. Kashif ${ }^{173}$, G. Kasieczka ${ }^{58 b}$, R.D. Kass ${ }^{109}$, A. Kastanas ${ }^{13}$, M. Kataoka ${ }^{4}$, Y. Kataoka ${ }^{155}$, E. Katsoufis ${ }^{9}$, J. Katzy ${ }^{41}$, V. Kaushik ${ }^{6}$, K. Kawagoe ${ }^{69}$, T. Kawamoto ${ }^{155}$, G. Kawamura ${ }^{81}$, M.S. Kay ${ }^{105}$, V.A. Kazanin ${ }^{107}$, M.Y. Kazarinov ${ }^{64}$, R. Keeler ${ }^{169}$, R. Kehoe ${ }^{39}$, M. Keil ${ }^{54}$, G.D. Kekelidze ${ }^{64}$, J.S. Keller ${ }^{138}$, M. Kenyon ${ }^{53}$, O. Kepka ${ }^{125}$, N. Kerschen ${ }^{29}$, B.P. Kerševan ${ }^{74}$, S. Kersten ${ }^{175}$, K. Kessoku ${ }^{155}$, J. Keung ${ }^{158}$, F. Khalil-zada ${ }^{10}$, H. Khandanyan ${ }^{165}$, A. Khanov ${ }^{112}$, D. Kharchenko ${ }^{64}$, A. Khodinov ${ }^{96}$, A. Khomich ${ }^{58 a}$, T.J. Khoo ${ }^{27}$, G. Khoriauli ${ }^{20}$,

A. Khoroshilov ${ }^{175}$, V. Khovanskiy ${ }^{95}$, E. Khramov ${ }^{64}$, J. Khubua ${ }^{51 b}$, H. Kim ${ }^{146 a, 146 b}$, S.H. Kim ${ }^{160}$, N. Kimura ${ }^{171}$, O. Kind ${ }^{15}$, B.T. King ${ }^{73}$, M. King ${ }^{66}$, R.S.B. King ${ }^{118}$, J. Kirk ${ }^{129}$, A.E. Kiryunin ${ }^{99}$, T. Kishimoto ${ }^{66}$, D. Kisielewska ${ }^{37}$, T. Kittelmann ${ }^{123}$, E. Kladiva ${ }^{144 \mathrm{~b}}$, M. Klein ${ }^{73}$, U. Klein ${ }^{73}$, K. Kleinknecht ${ }^{81}$, M. Klemetti ${ }^{85}$, A. Klier ${ }^{172}$, P. Klimek ${ }^{146 a, 146 b}$, A. Klimentov ${ }^{24}$, R. Klingenberg ${ }^{42}$, J.A. Klinger ${ }^{82}$, E.B. Klinkby ${ }^{35}$, T. Klioutchnikova ${ }^{29}$, P.F. Klok ${ }^{104}$, S. Klous ${ }^{105}$, E.-E. Kluge ${ }^{58 a}$, T. Kluge ${ }^{73}$, P. Kluit ${ }^{105}$, S. Kluth ${ }^{99}$, N.S. Knecht ${ }^{158}$, E. Kneringer ${ }^{61}$, E.B.F.G. Knoops ${ }^{83}$, A. Knue ${ }^{54}$, B.R. $\mathrm{Ko}^{44}$, T. Kobayashi ${ }^{155}$, M. Kobel ${ }^{43}$, M. Kocian ${ }^{143}$, P. Kodys ${ }^{126}$, K. Köneke ${ }^{29}$, A.C. König ${ }^{104}$, S. Koenig ${ }^{81}$, L. Köpke ${ }^{81}$, F. Koetsveld ${ }^{104}$, P. Koevesarki ${ }^{20}$, T. Koffas ${ }^{28}$, E. Koffeman ${ }^{105}$, L.A. Kogan ${ }^{118}$, S. Kohlmann ${ }^{175}$, F. Kohn ${ }^{54}$, Z. Kohout ${ }^{127}$, T. Kohriki ${ }^{65}$, T. Koi ${ }^{143}$, G.M. Kolachev ${ }^{107}$, H. Kolanoski ${ }^{15}$, V. Kolesnikov ${ }^{64}$, I. Koletsou ${ }^{89 a}$, J. Koll ${ }^{88}$, M. Kollefrath ${ }^{48}$, A.A. Komar ${ }^{94}$, Y. Komori ${ }^{155}$, T. Kondo ${ }^{65}$, T. Kono ${ }^{41, s}$, A.I. Kononov ${ }^{48}$, R. Konoplich ${ }^{108, t}$, N. Konstantinidis ${ }^{77}$, S. Koperny ${ }^{37}$, K. Korcyl ${ }^{38}$, K. Kordas ${ }^{154}$, A. Korn ${ }^{118}$, A. Korol ${ }^{107}$, I. Korolkov ${ }^{11}$, E.V. Korolkova ${ }^{139}$, V.A. Korotkov ${ }^{128}$, O. Kortner ${ }^{99}$, S. Kortner ${ }^{99}$, V.V. Kostyukhinn ${ }^{20}$, S. Kotov ${ }^{99}$, V.M. Kotov ${ }^{64}$, A. Kotwal ${ }^{44}$, C. Kourkoumelis ${ }^{8}$, V. Kouskoura ${ }^{154}$, A. Koutsman ${ }^{159 a}$, R. Kowalewski ${ }^{169}$, T.Z. Kowalski ${ }^{37}$, W. Kozanecki ${ }^{136}$, A.S. Kozhin ${ }^{128}$, V. Kral ${ }^{127}$, V.A. Kramarenko ${ }^{97}$, G. Kramberger ${ }^{74}$, M.W. Krasny ${ }^{78}$, A. Krasznahorkay ${ }^{108}$, J. Kraus ${ }^{88}$, J.K. Kraus ${ }^{20}$, S. Kreiss ${ }^{108}$, F. Krejci ${ }^{127}$, J. Kretzschmar ${ }^{73}$, N. Krieger ${ }^{54}$, P. Krieger ${ }^{158}$, K. Kroeninger ${ }^{54}$, H. Kroha ${ }^{99}$, J. Kroll ${ }^{120}$, J. Kroseberg ${ }^{20}$, J. Krstic ${ }^{12 a}$, U. Kruchonak ${ }^{64}$, H. Krüger ${ }^{20}$, T. Kruker ${ }^{16}$, N. Krumnack ${ }^{63}$, Z.V. Krumshteyn ${ }^{64}$, A. Kruth ${ }^{20}$, T. Kubota ${ }^{86}$, S. Kuday ${ }^{3 a}$, S. Kuehn ${ }^{48}$, A. Kugel ${ }^{58 c}$, T. Kuhl ${ }^{41}$, D. Kuhn ${ }^{61}$, V. Kukhtin ${ }^{64}$, Y. Kulchitsky ${ }^{90}$, S. Kuleshov ${ }^{31 b}$, C. Kummer ${ }^{98}$, M. Kuna ${ }^{78}$, J. Kunkle ${ }^{120}$, A. Kupco ${ }^{125}$, 
H. Kurashige ${ }^{66}$, M. Kurata ${ }^{160}$, Y.A. Kurochkin ${ }^{90}$, V. Kus ${ }^{125}$, E.S. Kuwertz ${ }^{147}$, M. Kuze ${ }^{157}$, J. Kvita ${ }^{142}$, R. Kwee ${ }^{15}$, A. La Rosa ${ }^{49}$, L. La Rotonda ${ }^{36 a, 36 b}$, L. Labarga ${ }^{80}$, J. Labbe ${ }^{4}$, S. Lablak ${ }^{135 a}$, C. Lacasta ${ }^{167}$, F. Lacava ${ }^{132 a, 132 b}$, H. Lacker ${ }^{15}$, D. Lacour ${ }^{78}$, V.R. Lacuesta ${ }^{167}$, E. Ladygin ${ }^{64}$, R. Lafaye ${ }^{4}$, B. Laforge ${ }^{78}$, T. Lagouri $^{80}$, S. Lai ${ }^{48}$, E. Laisne ${ }^{55}$, M. Lamanna ${ }^{29}$, L. Lambourne ${ }^{77}$, C.L. Lampen ${ }^{6}$, W. Lampl ${ }^{6}$, E. Lancon $^{136}$, U. Landgraf ${ }^{48}$, M.P.J. Landon ${ }^{75}$, J.L. Lane ${ }^{82}$, V.S. Lang ${ }^{58 a}$, C. Lange ${ }^{41}$, A.J. Lankford ${ }^{163}$, F. Lanni ${ }^{24}$, K. Lantzsch ${ }^{175}$, S. Laplace ${ }^{78}$, C. Lapoire ${ }^{20}$, J.F. Laporte ${ }^{136}$, T. Lari ${ }^{89 a}$, A. Larner ${ }^{118}$, M. Lassnig ${ }^{29}$, P. Laurelli ${ }^{47}$, V. Lavorini ${ }^{36 a, 36 b}$, W. Lavrijsen ${ }^{14}$, P. Laycock ${ }^{73}$, O. Le Dortz ${ }^{78}$, E. Le Guirriec ${ }^{83}$, C. Le Maner ${ }^{158}$, E. Le Menedeu ${ }^{11}$, T. LeCompte ${ }^{5}$, F. Ledroit-Guillon ${ }^{55}$, H. Lee ${ }^{105}$, J.S.H. Lee ${ }^{116}$, S.C. Lee ${ }^{151}$, L. Lee ${ }^{176}$, M. Lefebvre ${ }^{169}$, M. Legendre ${ }^{136}$, F. Legger ${ }^{98}$, C. Leggett ${ }^{14}$, M. Lehmacher $^{20}$, G. Lehmann Miotto ${ }^{29}$, X. Lei ${ }^{6}$, M.A.L. Leite ${ }^{23 d}$, R. Leitner ${ }^{126}$, D. Lellouch ${ }^{172}$, B. Lemmer ${ }^{54}$, V. Lendermann ${ }^{58 a}$, K.J.C. Leney ${ }^{145 b}$, T. Lenz ${ }^{105}$, G. Lenzen ${ }^{175}$, B. Lenzi ${ }^{29}$, K. Leonhardt ${ }^{43}$, S. Leontsinis ${ }^{9}$, F. Lepold ${ }^{58 a}$, C. Leroy ${ }^{93}$, J-R. Lessard ${ }^{169}$, C.G. Lester ${ }^{27}$, C.M. Lester ${ }^{120}$, J. Levêque ${ }^{4}$, D. Levin ${ }^{87}$, L.J. Levinson ${ }^{172}$, A. Lewis ${ }^{118}$, G.H. Lewis ${ }^{108}$, A.M. Leyko ${ }^{20}$, M. Leyton ${ }^{15}$, B. Li ${ }^{83}$, H. Li ${ }^{173, u}$, S. Li ${ }^{32 b, v}$, X. Li ${ }^{87}$, Z. Liang ${ }^{118, w}$, H. Liao ${ }^{33}$, B. Liberti ${ }^{133 a}$,

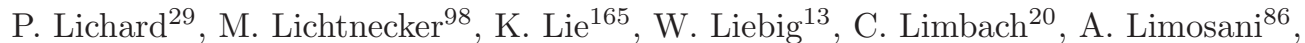
M. Limper 62 , S.C. $\operatorname{Lin}^{151, x}$, F. Linde ${ }^{105}$, J.T. Linnemann ${ }^{88}$, E. Lipeles ${ }^{120}$, A. Lipniacka ${ }^{13}$, T.M. Liss ${ }^{165}$, D. Lissauer ${ }^{24}$, A. Lister ${ }^{49}$, A.M. Litke ${ }^{137}$, C. Liu ${ }^{28}$, D. Liu ${ }^{151}$, H. Liu ${ }^{87}$, J.B. Liu ${ }^{87}$,

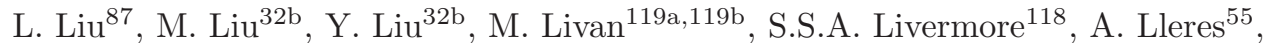
J. Llorente Merino ${ }^{80}$, S.L. Lloyd ${ }^{75}$, E. Lobodzinska ${ }^{41}$, P. Loch ${ }^{6}$, W.S. Lockman ${ }^{137}$, T. Loddenkoetter ${ }^{20}$, F.K. Loebinger ${ }^{82}$, A. Loginov ${ }^{176}$, C.W. Loh ${ }^{168}$, T. Lohse ${ }^{15}$, K. Lohwasser ${ }^{48}$, M. Lokajicek ${ }^{125}$, V.P. Lombardo ${ }^{4}$, R.E. Long ${ }^{71}$, L. Lopes ${ }^{124 a}$, D. Lopez Mateos ${ }^{57}$, J. Lorenz ${ }^{98}$, N. Lorenzo Martinez ${ }^{115}$, M. Losada ${ }^{162}$, P. Loscutoff ${ }^{14}$, F. Lo Sterzo ${ }^{132 a, 132 b}$, M.J. Losty ${ }^{159 a}$,

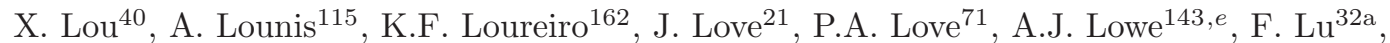
H.J. Lubatti ${ }^{138}$, C. Luci ${ }^{132 a, 132 b}$, A. Lucotte ${ }^{55}$, A. Ludwig ${ }^{43}$, D. Ludwig ${ }^{41}$, I. Ludwig ${ }^{48}$, J. Ludwig ${ }^{48}$, F. Luehring ${ }^{60}$, G. Luijckx ${ }^{105}$, W. Lukas ${ }^{61}$, D. Lumb ${ }^{48}$, L. Luminari ${ }^{132 a}$, E. Lund ${ }^{117}$, B. Lund-Jensen ${ }^{147}$, B. Lundberg ${ }^{79}$, J. Lundberg 146a,146b , O. Lundberg146a,146b, J. Lundquist ${ }^{35}$, M. Lungwitz ${ }^{81}$, D. Lynn ${ }^{24}$, E. Lytken ${ }^{79}$, H. Ma ${ }^{24}$, L.L. Ma ${ }^{173}$, G. Maccarrone ${ }^{47}$, A. Macchiolo ${ }^{99}$, B. Maček ${ }^{74}$, J. Machado Miguens ${ }^{124 a}$, R. Mackeprang ${ }^{35}$, R.J. Madaras ${ }^{14}$, W.F. Mader ${ }^{43}$, R. Maenner ${ }^{58 c}$, T. Maeno ${ }^{24}$, P. Mättig ${ }^{175}$, S. Mättig ${ }^{41}$, L. Magnoni ${ }^{29}$, E. Magradze $^{54}$, K. Mahboubi ${ }^{48}$, S. Mahmoud ${ }^{73}$, G. Mahout ${ }^{17}$, C. Maiani ${ }^{136}$, C. Maidantchik ${ }^{23 a}$, A. Maio ${ }^{124 a, b}$, S. Majewski ${ }^{24}$, Y. Makida ${ }^{65}$, N. Makovec ${ }^{115}$, P. Mal $^{136}$, B. Malaescu ${ }^{29}$, Pa. Malecki $^{38}$, P. Malecki ${ }^{38}$, V.P. Maleev ${ }^{121}$, F. Malek ${ }^{55}$, U. Mallik ${ }^{62}$, D. Malon ${ }^{5}$, C. Malone ${ }^{143}$, S. Maltezos ${ }^{9}$, V. Malyshev ${ }^{107}$, S. Malyukov ${ }^{29}$, R. Mameghani ${ }^{98}$, J. Mamuzic ${ }^{12 b}$, A. Manabe ${ }^{65}$, L. Mandelli ${ }^{89 a}$, I. Mandić ${ }^{74}$, R. Mandrysch ${ }^{15}$, J. Maneira ${ }^{124 a}$, P.S. Mangeard ${ }^{88}$, L. Manhaes de Andrade Filho ${ }^{23 a}$, A. Mann ${ }^{54}$, P.M. Manning ${ }^{137}$, A. Manousakis-Katsikakis ${ }^{8}$, B. Mansoulie ${ }^{136}$, A. Mapelli29, L. Mapelli ${ }^{29}$, L. March $^{80}$, J.F. Marchand ${ }^{28}$, F. Marchese ${ }^{133 a, 133 b}$, G. Marchiori ${ }^{78}$, M. Marcisovsky ${ }^{125}$, C.P. Marino ${ }^{169}$, F. Marroquim ${ }^{23 a}$, Z. Marshall ${ }^{29}$, F.K. Martens ${ }^{158}$,

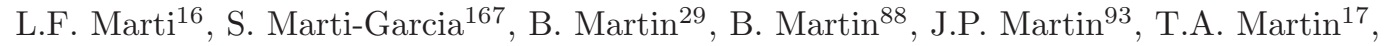
V.J. Martin ${ }^{45}$, B. Martin dit Latour ${ }^{49}$, S. Martin-Haugh ${ }^{149}$, M. Martinez ${ }^{11}$, V. Martinez Outschoorn ${ }^{57}$, A.C. Martyniuk ${ }^{169}$, M. Marx ${ }^{82}$, F. Marzano ${ }^{132 a}$, A. Marzin ${ }^{111}$, L. Masetti ${ }^{81}$, T. Mashimo ${ }^{155}$, R. Mashinistov ${ }^{94}$, J. Masik ${ }^{82}$, A.L. Maslennikov ${ }^{107}$, I. Massa ${ }^{19 a, 19 b}$, G. Massaro ${ }^{105}$, N. Massol ${ }^{4}$, A. Mastroberardino ${ }^{36 a, 36 b}$, T. Masubuchi ${ }^{155}$, P. Matricon ${ }^{115}$, H. Matsunaga ${ }^{155}$, T. Matsushita ${ }^{66}$, C. Mattravers ${ }^{118, c}$, J. Maurer ${ }^{83}$, S.J. Maxfield ${ }^{73}$, A. Mayne ${ }^{139}$, R. Mazini ${ }^{151}$, M. Mazur ${ }^{20}$, L. Mazzaferro ${ }^{133 a, 133 b}$, M. Mazzanti ${ }^{89 a}$, S.P. Mc Kee ${ }^{87}$, A. McCarn ${ }^{165}$, R.L. McCarthy ${ }^{148}$, T.G. McCarthy ${ }^{28}$, N.A. McCubbin ${ }^{129}$, K.W. McFarlane ${ }^{56}$, J.A. Mcfayden ${ }^{139}$, H. McGlone ${ }^{53}$, G. Mchedlidze ${ }^{51 b}$, T. Mclaughlan ${ }^{17}$, S.J. McMahon ${ }^{129}$, R.A. McPherson ${ }^{169, k}$, A. Meade ${ }^{84}$, J. Mechnich ${ }^{105}$, M. Mechtel ${ }^{175}$, M. Medinnis $^{41}$, R. Meera-Lebbai ${ }^{111}$, T. Meguro ${ }^{116}$, 
R. Mehdiyev ${ }^{93}$, S. Mehlhase ${ }^{35}$, A. Mehta ${ }^{73}$, K. Meier ${ }^{58 a}$, B. Meirose ${ }^{79}$, C. Melachrinos ${ }^{30}$, B.R. Mellado Garcia ${ }^{173}$, F. Meloni ${ }^{89 a, 89 b}$, L. Mendoza Navas ${ }^{162}$, Z. Meng ${ }^{151, u}$,

A. Mengarelli19a,19b ${ }^{19}$ S. Menke ${ }^{99}$, E. Meoni ${ }^{161}$, K.M. Mercurio ${ }^{57}$, P. Mermod $^{49}$, L. Merola ${ }^{102 a, 102 b}$,

C. Meroni ${ }^{89 a}$, F.S. Merritt ${ }^{30}$, H. Merritt ${ }^{109}$, A. Messina $^{29, y}$, J. Metcalfe ${ }^{103}$, A.S. Mete ${ }^{163}$,

C. Meyer $^{81}$, C. Meyer ${ }^{30}$, J-P. Meyer ${ }^{136}$, J. Meyer ${ }^{174}$, J. Meyer ${ }^{54}$, T.C. Meyer ${ }^{29}$, W.T. Meyer ${ }^{63}$,

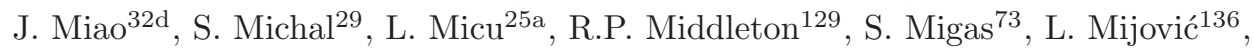

G. Mikenberg ${ }^{172}$, M. Mikestikova ${ }^{125}$, M. Mikuž ${ }^{74}$, D.W. Miller ${ }^{30}$, R.J. Miller ${ }^{88}$, W.J. Mills ${ }^{168}$,

C. Mills $^{57}$, A. Milov ${ }^{172}$, D.A. Milstead ${ }^{146 a, 146 b}$, D. Milstein ${ }^{172}$, A.A. Minaenko ${ }^{128}$, M. Miñano

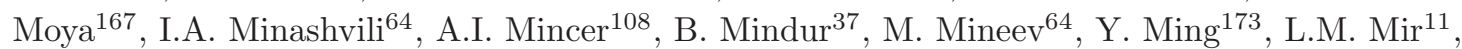

G. Mirabelli132a, J. Mitrevski ${ }^{137}$, V.A. Mitsou ${ }^{167}$, S. Mitsui ${ }^{65}$, P.S. Miyagawa ${ }^{139}$,

J.U. Mjörnmark ${ }^{79}$, T. Moa ${ }^{146 a, 146 b}$, V. Moeller ${ }^{27}$, K. Mönig ${ }^{41}$, N. Möser ${ }^{20}$, S. Mohapatra ${ }^{148}$,

W. Mohr ${ }^{48}$, R. Moles-Valls ${ }^{167}$, J. Monk ${ }^{77}$, E. Monnier ${ }^{83}$, J. Montejo Berlingen ${ }^{11}$,

S. Montesano ${ }^{89 a, 89 b}$, F. Monticelli ${ }^{70}$, S. Monzani ${ }^{19 a, 19 b}$, R.W. Moore ${ }^{2}$, G.F. Moorhead ${ }^{86}$,

C. Mora Herrera ${ }^{49}$, A. Moraes ${ }^{53}$, N. Morange ${ }^{136}$, J. Morel ${ }^{54}$, G. Morello ${ }^{36 a, 36 b}$, D. Moreno ${ }^{81}$,

M. Moreno Llácer ${ }^{167}$, P. Morettini ${ }^{50 a}$, M. Morgenstern ${ }^{43}$, M. Morii ${ }^{57}$, A.K. Morley ${ }^{29}$,

G. Mornacchi ${ }^{29}$, J.D. Morris ${ }^{75}$, L. Morvaj ${ }^{101}$, H.G. Moser ${ }^{99}$, M. Mosidze ${ }^{51 b}$, J. Moss ${ }^{109}$,

R. Mount ${ }^{143}$, E. Mountricha ${ }^{9, z}$, S.V. Mouraviev ${ }^{94}$, E.J.W. Moyse ${ }^{84}$, F. Mueller ${ }^{58 a}$, J. Mueller ${ }^{123}$,

K. Mueller ${ }^{20}$, T.A. Müller ${ }^{98}$, T. Mueller ${ }^{81}$, D. Muenstermann ${ }^{29}$, Y. Munwes ${ }^{153}$, W.J. Murray ${ }^{129}$,

I. Mussche ${ }^{105}$, E. Musto102a,102b, A.G. Myagkov ${ }^{128}$, M. Myska ${ }^{125}$, J. Nadal ${ }^{11}$, K. Nagai ${ }^{160}$,

K. Nagano ${ }^{65}$, A. Nagarkar ${ }^{109}$, Y. Nagasaka ${ }^{59}$, M. Nagel ${ }^{99}$, A.M. Nairz ${ }^{29}$, Y. Nakahama ${ }^{29}$,

K. Nakamura ${ }^{155}$, T. Nakamura ${ }^{155}$, I. Nakano ${ }^{110}$, G. Nanava ${ }^{20}$, A. Napier ${ }^{161}$, R. Narayan ${ }^{58 b}$,

M. Nash ${ }^{77, c}$, T. Nattermann ${ }^{20}$, T. Naumann ${ }^{41}$, G. Navarro ${ }^{162}$, H.A. Neal ${ }^{87}$, P.Yu. Nechaeva ${ }^{94}$,

T.J. Neep ${ }^{82}$, A. Negri ${ }^{119 a, 119 b}$, G. Negri ${ }^{29}$, S. Nektarijevic ${ }^{49}$, A. Nelson ${ }^{163}$, T.K. Nelson ${ }^{143}$,

S. Nemecek ${ }^{125}$, P. Nemethy ${ }^{108}$, A.A. Nepomuceno ${ }^{23 a}$, M. Nessi ${ }^{29, a a}$, M.S. Neubauer ${ }^{165}$,

A. Neusiedl ${ }^{81}$, R.M. Neves ${ }^{108}$, P. Nevski ${ }^{24}$, P.R. Newman ${ }^{17}$, V. Nguyen Thi Hong ${ }^{136}$,

R.B. Nickerson ${ }^{118}$, R. Nicolaidou ${ }^{136}$, B. Nicquevert ${ }^{29}$, F. Niedercorn ${ }^{115}$, J. Nielsen ${ }^{137}$,

N. Nikiforou ${ }^{34}$, A. Nikiforov ${ }^{15}$, V. Nikolaenko ${ }^{128}$, I. Nikolic-Audit ${ }^{78}$, K. Nikolics ${ }^{49}$,

K. Nikolopoulos ${ }^{24}$, H. Nilsen ${ }^{48}$, P. Nilsson ${ }^{7}$, Y. Ninomiya ${ }^{155}$, A. Nisati ${ }^{132 a}$, R. Nisius ${ }^{99}$,

T. Nobe ${ }^{157}$, L. Nodulman ${ }^{5}$, M. Nomachi ${ }^{116}$, I. Nomidis $^{154}$, M. Nordberg ${ }^{29}$, P.R. Norton ${ }^{129}$,

J. Novakova ${ }^{126}$, M. Nozaki ${ }^{65}$, L. Nozka ${ }^{113}$, I.M. Nugent ${ }^{159 a}$, A.-E. Nuncio-Quiroz ${ }^{20}$,

G. Nunes Hanninger ${ }^{86}$, T. Nunnemann ${ }^{98}$, E. Nurse ${ }^{77}$, B.J. O'Brien ${ }^{45}$, S.W. O'Neale ${ }^{17, *}$,

D.C. O'Neil ${ }^{142}$, V. O'Shea ${ }^{53}$, L.B. Oakes ${ }^{98}$, F.G. Oakham ${ }^{28, d}$, H. Oberlack ${ }^{99}$, J. Ocariz ${ }^{78}$,

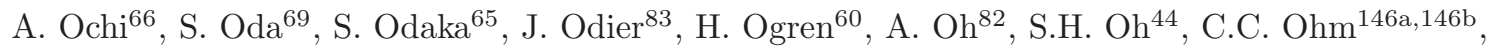

T. Ohshima ${ }^{101}$, H. Okawa ${ }^{163}$, Y. Okumura ${ }^{30}$, T. Okuyama ${ }^{155}$, A. Olariu ${ }^{25 a}$, A.G. Olchevski ${ }^{64}$,

S.A. Olivares Pino ${ }^{31 a}$, M. Oliveira ${ }^{124 a}, h$, D. Oliveira Damazio ${ }^{24}$, E. Oliver Garcia ${ }^{167}$,

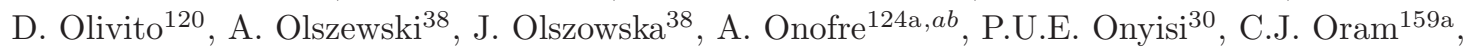

M.J. Oreglia ${ }^{30}$, Y. Oren ${ }^{153}$, D. Orestano ${ }^{134 a, 134 b}$, N. Orlando ${ }^{72 a, 72 b}$, I. Orlov ${ }^{107}$,

C. Oropeza Barrera ${ }^{53}$, R.S. Orr ${ }^{158}$, B. Osculati ${ }^{50 a, 50 b}$, R. Ospanov ${ }^{120}$, C. Osuna ${ }^{11}$,

G. Otero y Garzon ${ }^{26}$, J.P. Ottersbach ${ }^{105}$, M. Ouchrif ${ }^{135 d}$, E.A. Ouellette ${ }^{169}$, F. Ould-Saada ${ }^{117}$,

A. Ouraou ${ }^{136}$, Q. Ouyang ${ }^{32 a}$, A. Ovcharova ${ }^{14}$, M. Owen ${ }^{82}$, S. Owen ${ }^{139}$, V.E. Ozcan ${ }^{18 a}$,

N. Ozturk ${ }^{7}$, A. Pacheco Pages ${ }^{11}$, C. Padilla Aranda ${ }^{11}$, S. Pagan Griso ${ }^{14}$, E. Paganis ${ }^{139}$,

F. Paige ${ }^{24}$, P. Pais ${ }^{84}$, K. Pajchel ${ }^{117}$, G. Palacino ${ }^{159 b}$, C.P. Paleari ${ }^{6}$, S. Palestini ${ }^{29}$, D. Pallin ${ }^{33}$,

A. Palma ${ }^{124 a}$, J.D. Palmer ${ }^{17}$, Y.B. Pan $^{173}$, E. Panagiotopoulou ${ }^{9}$, P. Pani ${ }^{105}$, N. Panikashvili ${ }^{87}$,

S. Panitkin ${ }^{24}$, D. Pantea ${ }^{25 a}$, A. Papadelis ${ }^{146 a}$, Th.D. Papadopoulou ${ }^{9}$, A. Paramonov ${ }^{5}$,

D. Paredes Hernandez ${ }^{33}$, W. Park ${ }^{24, a c}$, M.A. Parker ${ }^{27}$, F. Parodi ${ }^{50 a, 50 b}$, J.A. Parsons ${ }^{34}$,

U. Parzefall ${ }^{48}$, S. Pashapour ${ }^{54}$, E. Pasqualucci132a , S. Passaggio ${ }^{50 a}$, A. Passeri ${ }^{134 a}$,

F. Pastore ${ }^{134 a, 134 b}$, Fr. Pastore ${ }^{76}$, G. Pásztor ${ }^{49, a d}$, S. Pataraia ${ }^{175}$, N. Patel ${ }^{150}$, J.R. Pater ${ }^{82}$,

S. Patricelli102a,102b, T. Pauly ${ }^{29}$, M. Pecsy ${ }^{144 a}$, M.I. Pedraza Morales ${ }^{173}$, S.V. Peleganchuk ${ }^{107}$, 
D. Pelikan ${ }^{166}$, H. Peng ${ }^{32 b}$, B. Penning ${ }^{30}$, A. Penson ${ }^{34}$, J. Penwell ${ }^{60}$, M. Perantoni23a, K. Perez ${ }^{34, a e}$, T. Perez Cavalcanti ${ }^{41}$, E. Perez Codina ${ }^{159 a}$, M.T. Pérez García-Estañ ${ }^{167}$, V. Perez Reale ${ }^{34}$, L. Perini ${ }^{89 a}, 89 b$, H. Pernegger ${ }^{29}$, R. Perrino ${ }^{72 a}$, P. Perrodo ${ }^{4}$, V.D. Peshekhonov ${ }^{64}$, K. Peters ${ }^{29}$, B.A. Petersen ${ }^{29}$, J. Petersen ${ }^{29}$, T.C. Petersen ${ }^{35}$, E. Petit ${ }^{4}$, A. Petridis ${ }^{154}$, C. Petridou ${ }^{154}$, E. Petrolo ${ }^{132 a}$, F. Petrucci ${ }^{134 a, 134 b}$, D. Petschull ${ }^{41}$, M. Petteni $^{142}$, R. Pezoa $^{31 b}$, A. Phan $^{86}$, P.W. Phillips ${ }^{129}$, G. Piacquadio ${ }^{29}$, A. Picazio ${ }^{49}$, E. Piccaro ${ }^{75}$, M. Piccinini ${ }^{19 a, 19 b}$, S.M. Piec ${ }^{41}$, R. Piegaia ${ }^{26}$, D.T. Pignotti ${ }^{109}$, J.E. Pilcher ${ }^{30}$, A.D. Pilkington ${ }^{82}$, J. Pina ${ }^{124 a, b}$, M. Pinamonti ${ }^{164 a, 164 c}$, A. Pinder ${ }^{118}$, J.L. Pinfold ${ }^{2}$, B. Pinto ${ }^{124 a}$, C. Pizio ${ }^{89 a, 89 b}$, M. Plamondon ${ }^{169}$, M.-A. Pleier ${ }^{24}$, E. Plotnikova ${ }^{64}$, A. Poblaguev ${ }^{24}$, S. Poddar ${ }^{58 a}$, F. Podlyski ${ }^{33}$, L. Poggioli ${ }^{115}$, T. Poghosyan ${ }^{20}$, M. Pohl ${ }^{49}$, G. Polesello ${ }^{119 a}$, A. Policicchio ${ }^{36 a}, 36 b$, A. Polini ${ }^{19 a}$, J. Poll ${ }^{75}$, V. Polychronakos ${ }^{24}$, D. Pomeroy ${ }^{22}$, K. Pommès ${ }^{29}$, L. Pontecorvo ${ }^{132 a}$, B.G. Pope ${ }^{88}$, G.A. Popeneciu ${ }^{25 a}$, D.S. Popovic ${ }^{12 a}$, A. Poppleton ${ }^{29}$, X. Portell Bueso ${ }^{29}$, G.E. Pospelov ${ }^{99}$, S. Pospisil ${ }^{127}$, I.N. Potrap ${ }^{99}$, C.J. Potter ${ }^{149}$, C.T. Potter ${ }^{114}$, G. Poulard ${ }^{29}$, J. Poveda ${ }^{60}$, V. Pozdnyakov ${ }^{64}$, R. Prabhu ${ }^{77}$, P. Pralavorio ${ }^{83}$, A. Pranko ${ }^{14}$, S. Prasad ${ }^{29}$, R. Pravahan ${ }^{24}$, S. Prell ${ }^{63}$, K. Pretzl ${ }^{16}$, D. Price $^{60}$, J. Price ${ }^{73}$, L.E. Price ${ }^{5}$, D. Prieur ${ }^{123}$, M. Primavera ${ }^{72 a}$, K. Prokofiev ${ }^{108}$, F. Prokoshin ${ }^{31 b}$, S. Protopopescu ${ }^{24}$, J. Proudfoot ${ }^{5}$, X. Prudent ${ }^{43}$, M. Przybycien ${ }^{37}$, H. Przysiezniak ${ }^{4}$, S. Psoroulas ${ }^{20}$, E. Ptacek ${ }^{114}$, E. Pueschel ${ }^{84}$, J. Purdham ${ }^{87}$, M. Purohit ${ }^{24, a c}$, P. Puzo ${ }^{115}$, Y. Pylypchenko ${ }^{62}$, J. Qian ${ }^{87}$, A. Quadt ${ }^{54}$, D.R. Quarrie ${ }^{14}$, W.B. Quayle ${ }^{173}$, F. Quinonez ${ }^{31 a}$, M. Raas ${ }^{104}$, V. Radescu ${ }^{41}$, P. Radloff ${ }^{114}$, T. Rador ${ }^{18 a}$, F. Ragusa ${ }^{89 a, 89 b}$, G. Rahal ${ }^{178}$, A.M. Rahimi ${ }^{109}$, D. Rahm ${ }^{24}$, S. Rajagopalan ${ }^{24}$, M. Rammensee ${ }^{48}$, M. Rammes ${ }^{141}$, A.S. Randle-Conde ${ }^{39}$, K. Randrianarivony ${ }^{28}$, F. Rauscher ${ }^{98}$, T.C. Rave ${ }^{48}$, M. Raymond ${ }^{29}$, A.L. Read ${ }^{117}$, D.M. Rebuzzi ${ }^{119 a, 119 b}$, A. Redelbach ${ }^{174}$, G. Redlinger ${ }^{24}$, R. Reece ${ }^{120}$, K. Reeves ${ }^{40}$, E. Reinherz-Aronis ${ }^{153}$, A. Reinsch ${ }^{114}$, I. Reisinger ${ }^{42}$, C. Rembser ${ }^{29}$, Z.L. $\operatorname{Ren}^{151}$, A. Renaud ${ }^{115}$, M. Rescigno ${ }^{132 a}$, S. Resconi ${ }^{89 a}$, B. Resende ${ }^{136}$, P. Reznicek ${ }^{98}$, R. Rezvani ${ }^{158}$, R. Richter ${ }^{99}$, E. Richter-Was ${ }^{4, a f}$, M. Ridel ${ }^{78}$, M. Rijpstra ${ }^{105}$, M. Rijssenbeek ${ }^{148}$, A. Rimoldi ${ }^{119 a, 119 b}$, L. Rinaldi ${ }^{19 a}$, R.R. Rios $^{39}$, I. Riu ${ }^{11}$, G. Rivoltella ${ }^{89 a, 89 b}$, F. Rizatdinova ${ }^{112}$, E. Rizvi ${ }^{75}$, S.H. Robertson ${ }^{85, k}$, A. Robichaud-Veronneau ${ }^{118}$, D. Robinson ${ }^{27}$, J.E.M. Robinson ${ }^{77}$, A. Robson ${ }^{53}$, J.G. Rocha de Lima ${ }^{106}$, C. Roda ${ }^{122 a, 122 b}$, D. Roda Dos Santos ${ }^{29}$, A. Roe ${ }^{54}$, S. Roe ${ }^{29}$, O. Røhne ${ }^{117}$, S. Rolli ${ }^{161}$, A. Romaniouk ${ }^{96}$, M. Romano ${ }^{19 a, 19 b}$, G. Romeo ${ }^{26}$, E. Romero Adam ${ }^{167}$, L. $\operatorname{Roos}^{78}$, E. Ros ${ }^{167}$, S. Rosati ${ }^{132 a}$, K. Rosbach ${ }^{49}$, A. Rose ${ }^{149}$, M. Rose ${ }^{76}$, G.A. Rosenbaum ${ }^{158}$, E.I. Rosenberg ${ }^{63}$, P.L. Rosendahl ${ }^{13}$, O. Rosenthal ${ }^{141}$, L. Rosselet $^{49}$, V. Rossetti ${ }^{11}$, E. Rossi ${ }^{132 a, 132 b}$, L.P. Rossi ${ }^{50 a}$, M. Rotaru ${ }^{25 a}$, I. Roth ${ }^{172}$, J. Rothberg ${ }^{138}$, D. Rousseau ${ }^{115}$, C.R. Royon ${ }^{136}$, A. Rozanov ${ }^{83}$, Y. Rozen ${ }^{152}$, X. Ruan ${ }^{32 a, a g}$, F. Rubbo ${ }^{11}$, I. Rubinskiy ${ }^{41}$, B. Ruckert ${ }^{98}$, N. Ruckstuhl ${ }^{105}$, V.I. Rud ${ }^{97}$, C. Rudolph ${ }^{43}$, G. Rudolph ${ }^{61}$, F. Rühr ${ }^{6}$, A. Ruiz-Martinez ${ }^{63}$, L. Rumyantsev ${ }^{64}$, Z. Rurikova ${ }^{48}$, N.A. Rusakovich ${ }^{64}$, J.P. Rutherfoord ${ }^{6}$, C. Ruwiedel ${ }^{14}$, P. Ruzicka ${ }^{125}$, Y.F. Ryabov ${ }^{121}$, P. Ryan ${ }^{88}$, M. Rybar ${ }^{126}$, G. Rybkin ${ }^{115}$, N.C. Ryder ${ }^{118}$, A.F. Saavedra ${ }^{150}$, I. Sadeh ${ }^{153}$, H.F-W. Sadrozinski ${ }^{137}$, R. Sadykov ${ }^{64}$, F. Safai Tehrani ${ }^{132 a}$, H. Sakamoto ${ }^{155}$, G. Salamanna ${ }^{75}$, A. Salamon ${ }^{133 a}$, M. Saleem ${ }^{111}$, D. Salek ${ }^{29}$, D. Salihagic ${ }^{99}$, A. Salnikov ${ }^{143}$, J. Salt ${ }^{167}$, B.M. Salvachua Ferrando ${ }^{5}$, D. Salvatore ${ }^{36 a, 36 b}$, F. Salvatore ${ }^{149}$, A. Salvucci ${ }^{104}$, A. Salzburger ${ }^{29}$, D. Sampsonidis ${ }^{154}$, B.H. Samset ${ }^{117}$, A. Sanchez ${ }^{102 a, 102 b}$, V. Sanchez Martinez ${ }^{167}$, H. Sandaker ${ }^{13}$, H.G. Sander ${ }^{81}$, M.P. Sanders ${ }^{98}$, M. Sandhoff ${ }^{175}$, T. Sandoval ${ }^{27}$, C. Sandoval ${ }^{162}$, R. Sandstroem ${ }^{99}$, D.P.C. Sankey ${ }^{129}$, A. Sansoni ${ }^{47}$, C. Santamarina Rios ${ }^{85}$, C. Santoni ${ }^{33}$, R. Santonico ${ }^{133 a, 133 b}$, H. Santos ${ }^{124 a}$, J.G. Saraiva ${ }^{124 a}$, T. Sarangi ${ }^{173}$, E. Sarkisyan-Grinbaum ${ }^{7}$, F. Sarri ${ }^{122 a, 122 b}$, G. Sartisohn ${ }^{175}$, O. Sasaki ${ }^{65}$, N. Sasao ${ }^{67}$, I. Satsounkevitch ${ }^{90}$, G. Sauvage ${ }^{4}$, E. Sauvan ${ }^{4}$, J.B. Sauvan ${ }^{115}$, P. Savard ${ }^{158, d}$, V. Savinov ${ }^{123}$, D.O. Savu ${ }^{29}$, L. Sawyer ${ }^{24, m}$, D.H. Saxon ${ }^{53}$, J. Saxon ${ }^{120}$, C. Sbarra ${ }^{19 a}$, A. Sbrizzi19a,19b, O. Scallon ${ }^{93}$, D.A. Scannicchio ${ }^{163}$, M. Scarcella ${ }^{150}$, J. Schaarschmidt ${ }^{115}$, P. Schacht ${ }^{99}$, D. Schaefer ${ }^{120}$, U. Schäfer ${ }^{81}$, S. Schaepe ${ }^{20}$, S. Schaetzel ${ }^{58 b}$, A.C. Schaffer ${ }^{115}$, D. Schaile ${ }^{98}$, 
R.D. Schamberger ${ }^{148}$, A.G. Schamov ${ }^{107}$, V. Scharf ${ }^{58 a}$, V.A. Schegelsky ${ }^{121}$, D. Scheirich $^{87}$, M. Schernau ${ }^{163}$, M.I. Scherzer ${ }^{34}$, C. Schiavi ${ }^{50 a, 50 b}$, J. Schieck ${ }^{98}$, M. Schioppa ${ }^{36 a, 36 b}$, S. Schlenker ${ }^{29}$, E. Schmidt ${ }^{48}$, K. Schmieden ${ }^{20}$, C. Schmitt ${ }^{81}$, S. Schmitt ${ }^{58 b}$, M. Schmitz ${ }^{20}$, B. Schneider ${ }^{16}$, U. Schnoor ${ }^{43}$, A. Schoening ${ }^{58 b}$, A.L.S. Schorlemmer ${ }^{54}$, M. Schott ${ }^{29}$, D. Schouten ${ }^{159 a}$, J. Schovancova ${ }^{125}$, M. Schram ${ }^{85}$, C. Schroeder ${ }^{81}$, N. Schroer ${ }^{58 c}$, M.J. Schultens ${ }^{20}$, J. Schultes ${ }^{175}$, H.-C. Schultz-Coulon ${ }^{58 a}$, H. Schulz ${ }^{15}$, M. Schumacher ${ }^{48}$, B.A. Schumm ${ }^{137}$, Ph. Schune ${ }^{136}$, C. Schwanenberger ${ }^{82}$, A. Schwartzman ${ }^{143}$, Ph. Schwemling ${ }^{78}$, R. Schwienhorst ${ }^{88}$, R. Schwierz ${ }^{43}$, J. Schwindling ${ }^{136}$, T. Schwindt ${ }^{20}$, M. Schwoerer ${ }^{4}$, G. Sciolla ${ }^{22}$, W.G. Scott ${ }^{129}$, J. Searcy ${ }^{114}$, G. Sedov ${ }^{41}$, E. Sedykh ${ }^{121}$, S.C. Seidel ${ }^{103}$, A. Seiden ${ }^{137}$, F. Seifert ${ }^{43}$, J.M. Seixas ${ }^{23 a}$, G. Sekhniaidze ${ }^{102 a}$, S.J. Sekula ${ }^{39}$, K.E. Selbach ${ }^{45}$, D.M. Seliverstov ${ }^{121}$, B. Sellden ${ }^{146 a}$, G. Sellers ${ }^{73}$, M. Seman ${ }^{144 b}$, N. Semprini-Cesari ${ }^{19 a, 19 b}$, C. Serfon ${ }^{98}$, L. Serin ${ }^{115}$, L. Serkin ${ }^{54}$, R. Seuster ${ }^{99}$, H. Severini ${ }^{111}$, A. Sfyrla ${ }^{29}$, E. Shabalina ${ }^{54}$, M. Shamim ${ }^{114}$, L.Y. Shan ${ }^{32 a}$, J.T. Shank ${ }^{21}$, Q.T. Shao ${ }^{86}$, M. Shapiro ${ }^{14}$, P.B. Shatalov ${ }^{95}$, K. Shaw ${ }^{164 a, 164 c}$, D. Sherman ${ }^{176}$, P. Sherwood ${ }^{77}$, A. Shibata ${ }^{108}$, S. Shimizu ${ }^{29}$, M. Shimojima ${ }^{100}$, T. Shin ${ }^{56}$, M. Shiyakova ${ }^{64}$, A. Shmeleva ${ }^{94}$, M.J. Shochet ${ }^{30}$, D. Short ${ }^{118}$, S. Shrestha ${ }^{63}$, E. Shulga ${ }^{96}$, M.A. Shupe ${ }^{6}$, P. Sicho ${ }^{125}$, A. Sidoti132a ${ }^{132}$ F. Siegert ${ }^{48}$, Dj. Sijacki ${ }^{12 a}$, O. Silbert ${ }^{172}$, J. Silva ${ }^{124 a}$, Y. Silver ${ }^{153}$, D. Silverstein ${ }^{143}$, S.B. Silverstein ${ }^{146 a}$, V. Simak ${ }^{127}$, O. Simard ${ }^{136}$, Lj. Simic ${ }^{12 a}$, S. Simion ${ }^{115}$, E. Simioni ${ }^{81}$, B. Simmons ${ }^{77}$, R. Simoniello ${ }^{89 a, 89 b}$, M. Simonyan ${ }^{35}$, P. Sinervo ${ }^{158}$, N.B. Sinev ${ }^{114}$, V. Sipica ${ }^{141}$, G. Siragusa ${ }^{174}$, A. Sircar ${ }^{24}$, A.N. Sisakyan ${ }^{64}$, S.Yu. Sivoklokov ${ }^{97}$, J. Sjölin ${ }^{146 a, 146 b}$, T.B. Sjursen ${ }^{13}$, L.A. Skinnari ${ }^{14}$, H.P. Skottowe ${ }^{57}$, K. Skovpen ${ }^{107}$, P. Skubic ${ }^{111}$, M. Slater ${ }^{17}$, T. Slavicek ${ }^{127}$, K. Sliwa ${ }^{161}$, V. Smakhtin ${ }^{172}$, B.H. Smart ${ }^{45}$, S.Yu. Smirnov ${ }^{96}$, Y. Smirnov ${ }^{96}$, L.N. Smirnova ${ }^{97}$, O. Smirnova ${ }^{79}$, B.C. Smith ${ }^{57}$, D. Smith ${ }^{143}$, K.M. Smith ${ }^{53}$, M. Smizanska ${ }^{71}$, K. Smolek ${ }^{127}$, A.A. Snesarev ${ }^{94}$, S.W. Snow ${ }^{82}$, J. Snow ${ }^{111}$, S. Snyder ${ }^{24}$, R. Sobie ${ }^{169, k}$, J. Sodomka ${ }^{127}$, A. Soffer ${ }^{153}$, C.A. Solans ${ }^{167}$, M. Solar ${ }^{127}$, J. Solc ${ }^{127}$, E.Yu. Soldatov ${ }^{96}$, U. Soldevila ${ }^{167}$, E. Solfaroli Camillocci ${ }^{132 a, 132 b}$, A.A. Solodkov ${ }^{128}$, O.V. Solovyanov ${ }^{128}$, N. Soni ${ }^{2}$, V. Sopko ${ }^{127}$, B. Sopko ${ }^{127}$, M. Sosebee ${ }^{7}$, R. Soualah ${ }^{164 a, 164 c}$, A. Soukharev ${ }^{107}$, S. Spagnolo72a,72b, F. Spanò ${ }^{76}$, R. Spighi19a , G. Spigo ${ }^{29}$, F. Spila132a,132b, R. Spiwoks ${ }^{29}$, M. Spousta ${ }^{126}$, T. Spreitzer ${ }^{158}$, B. Spurlock ${ }^{7}$, R.D. St. Denis ${ }^{53}$, J. Stahlman ${ }^{120}$, R. Stamen ${ }^{58 a}$, E. Stanecka ${ }^{38}$, R.W. Stanek ${ }^{5}$, C. Stanescu ${ }^{134 a}$, M. Stanescu-Bellu ${ }^{41}$, S. Stapnes ${ }^{117}$, E.A. Starchenko ${ }^{128}$, J. Stark ${ }^{55}$, P. Staroba ${ }^{125}$, P. Starovoitov ${ }^{41}$, R. Staszewski ${ }^{38}$, A. Staude ${ }^{98}$, P. Stavina ${ }^{144 a}$, G. Steele ${ }^{53}$, P. Steinbach ${ }^{43}$, P. Steinberg ${ }^{24}$, I. Stekl ${ }^{127}$, B. Stelzer ${ }^{142}$, H.J. Stelzer ${ }^{88}$, O. Stelzer-Chilton ${ }^{159 a}$, H. Stenzel ${ }^{52}$, S. Stern ${ }^{99}$, G.A. Stewart ${ }^{29}$, J.A. Stillings ${ }^{20}$, M.C. Stockton ${ }^{85}$, K. Stoerig ${ }^{48}$, G. Stoicea ${ }^{25 a}$, S. Stonjek ${ }^{99}$, P. Strachota ${ }^{126}$, A.R. Stradling ${ }^{7}$, A. Straessner ${ }^{43}$, J. Strandberg ${ }^{147}$, S. Strandberg ${ }^{146 a, 146 b}$, A. Strandlie ${ }^{117}$, M. Strang ${ }^{109}$, E. Strauss ${ }^{143}$, M. Strauss ${ }^{111}$, P. Strizenec ${ }^{144 b}$, R. Ströhmer ${ }^{174}$, D.M. Strom ${ }^{114}$, J.A. Strong ${ }^{76, *}$,

R. Stroynowski ${ }^{39}$, J. Strube ${ }^{129}$, B. Stugu ${ }^{13}$, I. Stumer ${ }^{24, *}$, J. Stupak ${ }^{148}$, P. Sturm ${ }^{175}$, N.A. Styles ${ }^{41}$, D.A. Soh ${ }^{151, w}$, D. Su ${ }^{143}$, HS. Subramania ${ }^{2}$, A. Succurro ${ }^{11}$, Y. Sugaya ${ }^{116}$, C. Suhr ${ }^{106}$, M. Suk ${ }^{126}$, V.V. Sulin ${ }^{94}$, S. Sultansoy ${ }^{3 d}$, T. Sumida ${ }^{67}$, X. Sun ${ }^{55}$, J.E. Sundermann ${ }^{48}$, K. Suruliz ${ }^{139}$, G. Susinno ${ }^{36 a, 36 b}$, M.R. Sutton ${ }^{149}$, Y. Suzuki ${ }^{65}$, Y. Suzuki ${ }^{66}$, M. Svatos ${ }^{125}$,

S. Swedish ${ }^{168}$, I. Sykora ${ }^{144 a}$, T. Sykora ${ }^{126}$, J. Sánchez ${ }^{167}$, D. Ta ${ }^{105}$, K. Tackmann ${ }^{41}$, A. Taffard ${ }^{163}$, R. Tafirout ${ }^{159 a}$, N. Taiblum ${ }^{153}$, Y. Takahashi ${ }^{101}$, H. Takai ${ }^{24}$, R. Takashima ${ }^{68}$, H. Takeda ${ }^{66}$, T. Takeshita ${ }^{140}$, Y. Takubo ${ }^{65}$, M. Talby ${ }^{83}$, A. Talyshev ${ }^{107, f}$, M.C. Tamsett ${ }^{24}$, J. Tanaka ${ }^{155}$, R. Tanaka ${ }^{115}$, S. Tanaka ${ }^{131}$, S. Tanaka ${ }^{65}$, A.J. Tanasijczuk ${ }^{142}$, K. Tani ${ }^{66}$, N. Tannoury ${ }^{83}$, S. Tapprogge ${ }^{81}$, D. Tardif ${ }^{158}$, S. Tarem ${ }^{152}$, F. Tarrade ${ }^{28}$, G.F. Tartarelli ${ }^{89 a}$, P. Tas ${ }^{126}$, M. Tasevsky ${ }^{125}$, E. Tassi ${ }^{36 a, 36 b}$, M. Tatarkhanov ${ }^{14}$, Y. Tayalati ${ }^{135 d}$, C. Taylor ${ }^{77}$, F.E. Taylor ${ }^{92}$, G.N. Taylor ${ }^{86}$, W. Taylor ${ }^{159 b}$, M. Teinturier ${ }^{115}$, M. Teixeira Dias Castanheira ${ }^{75}$,

P. Teixeira-Dias ${ }^{76}$, K.K. Temming ${ }^{48}$, H. Ten Kate ${ }^{29}$, P.K. Teng ${ }^{151}$, S. Terada ${ }^{65}$, K. Terashi ${ }^{155}$, J. Terron ${ }^{80}$, M. Testa ${ }^{47}$, R.J. Teuscher ${ }^{158, k}$, J. Therhaag ${ }^{20}$, T. Theveneaux-Pelzer ${ }^{78}$, S. Thoma ${ }^{48}$, 
J.P. Thomas ${ }^{17}$, E.N. Thompson ${ }^{34}$, P.D. Thompson ${ }^{17}$, P.D. Thompson ${ }^{158}$, A.S. Thompson ${ }^{53}$, L.A. Thomsen ${ }^{35}$, E. Thomson ${ }^{120}$, M. Thomson ${ }^{27}$, R.P. Thun ${ }^{87}$, F. $\operatorname{Tian}^{34}$, M.J. Tibbetts ${ }^{14}$, T. Tic $^{125}$, V.O. Tikhomirov ${ }^{94}$, Y.A. Tikhonov ${ }^{107, f}$, S. Timoshenko ${ }^{96}$, P. Tipton ${ }^{176}$, F.J. Tique Aires Viegas ${ }^{29}$, S. Tisserant ${ }^{83}$, T. Todorov ${ }^{4}$, S. Todorova-Nova ${ }^{161}$, B. Toggerson ${ }^{163}$, J. Tojo ${ }^{69}, \mathrm{~S}$. Tokár ${ }^{144 \mathrm{a}}$, K. Tokushuku ${ }^{65}, \mathrm{~K}$. Tollefson $^{88}$, M. Tomoto ${ }^{101}$, L. Tompkins ${ }^{30}$, K. Toms ${ }^{103}$, A. Tonoyan ${ }^{13}$, C. Topfel ${ }^{16}$, N.D. Topilin ${ }^{64}$, I. Torchiani ${ }^{29}$, E. Torrence ${ }^{114}$, H. Torres ${ }^{78}$, E. Torró Pastor ${ }^{167}$, J. Toth ${ }^{83, a d}$, F. Touchard ${ }^{83}$, D.R. Tovey ${ }^{139}$, T. Trefzger ${ }^{174}$, L. Tremblet ${ }^{29}$, A. Tricoli ${ }^{29}$, I.M. Trigger ${ }^{159 a}$, S. Trincaz-Duvoid ${ }^{78}$, M.F. Tripiana ${ }^{70}$, W. Trischuk ${ }^{158}$, B. Trocmé ${ }^{55}$, C. Troncon ${ }^{89 a}$, M. Trottier-McDonald ${ }^{142}$, M. Trzebinski $^{38}$, A. Trzupek $^{38}$, C. Tsarouchas ${ }^{29}$, J.C-L. Tseng ${ }^{118}$, M. Tsiakiris ${ }^{105}$, P.V. Tsiareshka ${ }^{90}$, D. Tsionou ${ }^{4, a h}$, G. Tsipolitis ${ }^{9}$, S. Tsiskaridze ${ }^{11}$, V. Tsiskaridze ${ }^{48}$, E.G. Tskhadadze ${ }^{51 a}$, I.I. Tsukerman ${ }^{95}$, V. Tsulaia ${ }^{14}$, J.-W. Tsung ${ }^{20}$, S. Tsuno ${ }^{65}$, D. Tsybychev ${ }^{148}$, A. Tua ${ }^{139}$, A. Tudorache ${ }^{25 a}$, V. Tudorache ${ }^{25 a}$, J.M. Tuggle ${ }^{30}$, M. Turala ${ }^{38}$, D. Turecek ${ }^{127}$, I. Turk Cakir ${ }^{3 e}$, E. Turlay ${ }^{105}$, R. Turra ${ }^{89 a, 89 b}$, P.M. Tuts ${ }^{34}$, A. Tykhonov ${ }^{74}$, M. Tylmad ${ }^{146 a, 146 b}$, M. Tyndel ${ }^{129}$,

G. Tzanakos ${ }^{8}$, K. Uchida ${ }^{20}$, I. Ueda ${ }^{155}$, R. Ueno ${ }^{28}$, M. Ugland ${ }^{13}$, M. Uhlenbrock ${ }^{20}$,

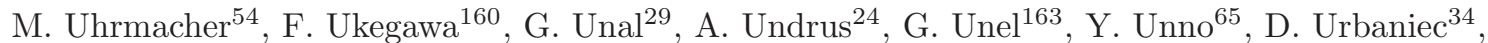
G. Usai ${ }^{7}$, M. Uslenghi ${ }^{119 a, 119 b}$, L. Vacavant ${ }^{83}$, V. Vacek ${ }^{127}$, B. Vachon ${ }^{85}$, S. Vahsen $^{14}$,

J. Valenta ${ }^{125}$, P. Valente ${ }^{132 a}$, S. Valentinetti19a,19b, A. Valero ${ }^{167}$, S. Valkar ${ }^{126}$,

E. Valladolid Gallego ${ }^{167}$, S. Vallecorsa ${ }^{152}$, J.A. Valls Ferrer ${ }^{167}$, H. van der Graaf ${ }^{105}$,

E. van der Kraaij ${ }^{105}$, R. Van Der Leeuw ${ }^{105}$, E. van der Poel ${ }^{105}$, D. van der Ster ${ }^{29}$, N. van Eldik ${ }^{29}$, P. van Gemmeren ${ }^{5}$, I. van Vulpen ${ }^{105}$, M. Vanadia ${ }^{99}$, W. Vandelli ${ }^{29}$, A. Vaniachine ${ }^{5}$, P. Vankov ${ }^{41}$, F. Vannucci ${ }^{78}$, R. Vari ${ }^{132 a}$, T. $\operatorname{Varol}^{84}$, D. Varouchas ${ }^{14}$, A. Vartapetian ${ }^{7}$, K.E. $\operatorname{Varvell}^{150}$, V.I. Vassilakopoulos ${ }^{56}$, F. Vazeille ${ }^{33}$, T. Vazquez Schroeder ${ }^{54}$, G. Vegni ${ }^{89 a, 89 b}$, J.J. Veillet ${ }^{115}$,

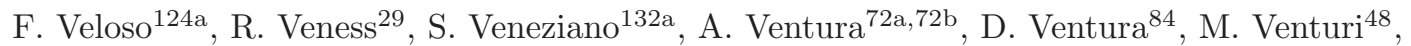
N. Venturi ${ }^{158}$, V. Vercesi ${ }^{119 a}$, M. Verducci ${ }^{138}$, W. Verkerke ${ }^{105}$, J.C. Vermeulen ${ }^{105}$, A. Vest ${ }^{43}$, M.C. Vetterli ${ }^{142, d}$, I. Vichou ${ }^{165}$, T. Vickey ${ }^{145 b, a i}$, O.E. Vickey Boeriu ${ }^{145 b}$, G.H.A. Viehhauser ${ }^{118}$, S. Viel ${ }^{168}$, M. Villa ${ }^{19 a, 19 b}$, M. Villaplana Perez ${ }^{167}$, E. Vilucchi ${ }^{47}$, M.G. Vincter ${ }^{28}$, E. Vinek $^{29}$, V.B. Vinogradov ${ }^{64}$, M. Virchaux ${ }^{136, *}$,J. Virzi ${ }^{14}$, O. Vitells ${ }^{172}$, M. Viti ${ }^{41}$, I. Vivarelli $^{48}$,

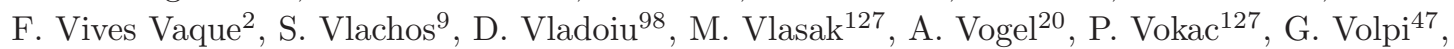
M. Volpi ${ }^{86}$, G. Volpini ${ }^{89 a}$, H. von der Schmitt ${ }^{99}$, J. von Loeben ${ }^{99}$, H. von Radziewski ${ }^{48}$, E. von Toerne ${ }^{20}, \mathrm{~V}$. Vorobel ${ }^{126}, \mathrm{~V}$. Vorwerk ${ }^{11}, \mathrm{M} . \operatorname{Vos}^{167}, \mathrm{R} . \operatorname{Voss}^{29}, \mathrm{~T} . \mathrm{T} . \operatorname{Voss}^{175}$, J.H. Vossebeld ${ }^{73}$, N. Vranjes ${ }^{136}$, M. Vranjes Milosavljevic ${ }^{105}$, V. Vrba ${ }^{125}$, M. Vreeswijk ${ }^{105}$, T. Vu Anh ${ }^{48}$, R. Vuillermet ${ }^{29}$, I. Vukotic ${ }^{115}$, W. Wagner ${ }^{175}$, P. Wagner ${ }^{120}$, H. Wahlen ${ }^{175}$, S. Wahrmund ${ }^{43}$, J. Wakabayashi ${ }^{101}$, S. Walch ${ }^{87}$, J. Walder ${ }^{71}$, R. Walker ${ }^{98}$, W. Walkowiak ${ }^{141}$, R. Wall ${ }^{176}$, P. Waller ${ }^{73}$, C. Wang ${ }^{44}$, H. Wang ${ }^{173}$, H. Wang ${ }^{32 b, a j}$, J. Wang ${ }^{151}$, J. Wang ${ }^{55}$, R. Wang ${ }^{103}$, S.M. Wang ${ }^{151}$, T. Wang ${ }^{20}$, A. Warburton ${ }^{85}$, C.P. Ward ${ }^{27}$, M. Warsinsky ${ }^{48}$, A. Washbrook ${ }^{45}$, C. Wasicki ${ }^{41}$, P.M. Watkins ${ }^{17}$, A.T. Watson ${ }^{17}$, I.J. Watson ${ }^{150}$, M.F. Watson ${ }^{17}$, G. Watts ${ }^{138}$, S. Watts ${ }^{82}$, A.T. Waugh ${ }^{150}$, B.M. Waugh ${ }^{77}$, M. Weber ${ }^{129}$, M.S. Weber ${ }^{16}$, P. Weber ${ }^{54}$, A.R. Weidberg ${ }^{118}$, P. Weigell ${ }^{99}$, J. Weingarten ${ }^{54}$, C. Weiser ${ }^{48}$, H. Wellenstein ${ }^{22}$, P.S. Wells ${ }^{29}$, T. Wenaus ${ }^{24}$, D. Wendland ${ }^{15}$, Z. Weng ${ }^{151, w}$, T. Wengler ${ }^{29}$, S. Wenig ${ }^{29}$, N. Wermes ${ }^{20}$, M. Werner ${ }^{48}$, P. Werner ${ }^{29}$, M. Werth ${ }^{163}$, M. Wessels ${ }^{58 a}$, J. Wetter ${ }^{161}$, C. Weydert ${ }^{55}$, K. Whalen ${ }^{28}$, S.J. Wheeler-Ellis ${ }^{163}$, A. White ${ }^{7}$, M.J. White ${ }^{86}$, S. White ${ }^{122 a, 122 b}$, S.R. Whitehead ${ }^{118}$,

D. Whiteson ${ }^{163}$, D. Whittington ${ }^{60}$, F. Wicek ${ }^{115}$, D. Wicke ${ }^{175}$, F.J. Wickens ${ }^{129}$,

W. Wiedenmann ${ }^{173}$, M. Wielers ${ }^{129}$, P. Wienemann ${ }^{20}$, C. Wiglesworth ${ }^{75}$, L.A.M. Wiik-Fuchs ${ }^{48}$, P.A. Wijeratne ${ }^{77}$, A. Wildauer ${ }^{167}$, M.A. Wildt ${ }^{41, s}$, I. Wilhelm ${ }^{126}$, H.G. Wilkens ${ }^{29}$, J.Z. Will ${ }^{98}$, E. Williams ${ }^{34}$, H.H. Williams ${ }^{120}$, W. Willis ${ }^{34}$, S. Willocq ${ }^{84}$, J.A. Wilson ${ }^{17}$, M.G. Wilson ${ }^{143}$, A. Wilson ${ }^{87}$, I. Wingerter-Seez ${ }^{4}$, S. Winkelmann ${ }^{48}$, F. Winklmeier ${ }^{29}$, M. Wittgen ${ }^{143}$,

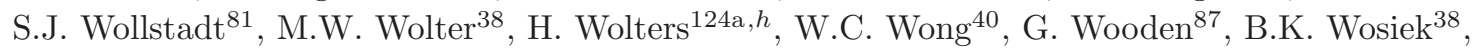


J. Wotschack ${ }^{29}$, M.J. Woudstra ${ }^{82}$, K.W. Wozniak ${ }^{38}$, K. Wraight ${ }^{53}$, C. Wright ${ }^{53}$, M. Wright ${ }^{53}$, B. Wrona ${ }^{73}$, S.L. Wu ${ }^{173}$, X. Wu ${ }^{49}$, Y. Wu ${ }^{32 b, a k}$, E. Wulf ${ }^{34}$, B.M. Wynne ${ }^{45}$, S. Xella ${ }^{35}$, M. Xiao ${ }^{136}$, S. Xie ${ }^{48}$, C. Xu ${ }^{32 b, z}$, D. Xu ${ }^{139}$, B. Yabsley ${ }^{150}$, S. Yacoob ${ }^{145 b}$, M. Yamada ${ }^{65}$, H. Yamaguchi ${ }^{155}$, A. Yamamoto ${ }^{65}$, K. Yamamoto ${ }^{63}$, S. Yamamoto ${ }^{155}$, T. Yamamura ${ }^{155}$, T. Yamanaka ${ }^{155}$, J. Yamaoka ${ }^{44}$, T. Yamazaki ${ }^{155}$, Y. Yamazaki ${ }^{66}$, Z. Yan ${ }^{21}$, H. Yang ${ }^{87}$, U.K. Yang ${ }^{82}$, Y. Yang ${ }^{60}$, Z. Yang ${ }^{146 a, 146 b}$, S. Yanush ${ }^{11}$, L. Yao ${ }^{32 a}$, Y. Yao ${ }^{14}$, Y. Yasu ${ }^{65}$, G.V. Ybeles Smit ${ }^{130}$, J. Ye ${ }^{39}$, S. Ye ${ }^{24}$, M. Yilmaz ${ }^{3 c}$, R. Yoosoofmiya ${ }^{123}$, K. Yorita ${ }^{171}$, R. Yoshida ${ }^{5}$, C. Young ${ }^{143}$, C.J. Young ${ }^{118}$, S. Youssef ${ }^{21}$, D. Yu ${ }^{24}$, J. Yu$^{7}$, J. Yu ${ }^{112}$, L. Yuan ${ }^{66}$, A. Yurkewicz ${ }^{106}$, B. Zabinski ${ }^{38}$, R. Zaidan ${ }^{62}$, A.M. Zaitsev ${ }^{128}$, Z. Zajacova ${ }^{29}$, L. Zanello ${ }^{132 a, 132 b}$, A. Zaytsev ${ }^{107}$, C. Zeitnitz ${ }^{175}$, M. Zeman ${ }^{125}$, A. Zemla ${ }^{38}$, C. Zendler ${ }^{20}$, O. Zenin ${ }^{128}$, T. Ženiš ${ }^{144 a}$, Z. Zinonos ${ }^{122 a, 122 b}$, S. Zenz ${ }^{14}$, D. Zerwas ${ }^{115}$, G. Zevi della Porta ${ }^{57}$, Z. Zhan ${ }^{32 d}$, D. Zhang ${ }^{32 \mathrm{~b}, a j}$, H. Zhang ${ }^{88}$, J. Zhang ${ }^{5}$, X. Zhang ${ }^{32 d}$,

Z. Zhang ${ }^{115}$, L. Zhao ${ }^{108}$, T. Zhao ${ }^{138}$, Z. Zhao ${ }^{32 b}$, A. Zhemchugov ${ }^{64}$, J. Zhong ${ }^{118}$, B. Zhou ${ }^{87}$, N. Zhou ${ }^{163}$, Y. Zhou ${ }^{151}$, C.G. Zhu ${ }^{32 d}$, H. Zhu ${ }^{41}$, J. Zhu ${ }^{87}$, Y. Zhu ${ }^{32 b}$, X. Zhuang ${ }^{98}$,

V. Zhuravlov ${ }^{99}$, D. Zieminska ${ }^{60}$, N.I. Zimin ${ }^{64}$, R. Zimmermann ${ }^{20}$, S. Zimmermann ${ }^{20}$,

S. Zimmermann ${ }^{48}$, M. Ziolkowski ${ }^{141}$, R. Zitoun ${ }^{4}$, L. Živković ${ }^{34}$, V.V. Zmouchko ${ }^{128, *}$,

G. Zobernig ${ }^{173}$, A. Zoccoli ${ }^{19 a, 19 b}$, M. zur Nedden ${ }^{15}$, V. Zutshi ${ }^{106}$ and L. Zwalinski ${ }^{29}$

1 University at Albany, Albany NY, United States of America

2 Department of Physics, University of Alberta, Edmonton AB, Canada

$3{ }^{(a)}$ Department of Physics, Ankara University, Ankara; ${ }^{(b)}$ Department of Physics, Dumlupinar University, Kutahya; ${ }^{(c)}$ Department of Physics, Gazi University, Ankara; ${ }^{(d)}$ Division of Physics, TOBB University of Economics and Technology, Ankara; ${ }^{(e)}$ Turkish Atomic Energy Authority, Ankara, Turkey

4 LAPP, CNRS/IN2P3 and Université de Savoie, Annecy-le-Vieux, France

5 High Energy Physics Division, Argonne National Laboratory, Argonne IL, United States of America

6 Department of Physics, University of Arizona, Tucson AZ, United States of America

7 Department of Physics, The University of Texas at Arlington, Arlington TX, United States of America

8 Physics Department, University of Athens, Athens, Greece

9 Physics Department, National Technical University of Athens, Zografou, Greece

10 Institute of Physics, Azerbaijan Academy of Sciences, Baku, Azerbaijan

11 Institut de Física d'Altes Energies and Departament de Física de la Universitat Autònoma de Barcelona and ICREA, Barcelona, Spain

$12{ }^{(a)}$ Institute of Physics, University of Belgrade, Belgrade; ${ }^{(b)}$ Vinca Institute of Nuclear Sciences, University of Belgrade, Belgrade, Serbia

13 Department for Physics and Technology, University of Bergen, Bergen, Norway

14 Physics Division, Lawrence Berkeley National Laboratory and University of California, Berkeley CA, United States of America

15 Department of Physics, Humboldt University, Berlin, Germany

16 Albert Einstein Center for Fundamental Physics and Laboratory for High Energy Physics, University of Bern, Bern, Switzerland

17 School of Physics and Astronomy, University of Birmingham, Birmingham, United Kingdom

$18{ }^{(a)}$ Department of Physics, Bogazici University, Istanbul; ${ }^{(b)}$ Division of Physics, Dogus University, Istanbul; ${ }^{(c)}$ Department of Physics Engineering, Gaziantep University, Gaziantep; ${ }^{(d)}$ Department of Physics, Istanbul Technical University, Istanbul, Turkey

$19{ }^{(a)}$ INFN Sezione di Bologna; ${ }^{(b)}$ Dipartimento di Fisica, Università di Bologna, Bologna, Italy

20 Physikalisches Institut, University of Bonn, Bonn, Germany 
21 Department of Physics, Boston University, Boston MA, United States of America

22 Department of Physics, Brandeis University, Waltham MA, United States of America

23 (a) Universidade Federal do Rio De Janeiro COPPE/EE/IF, Rio de Janeiro; ${ }^{(b)}$ Federal University of Juiz de Fora (UFJF), Juiz de Fora; ${ }^{(c)}$ Federal University of Sao Joao del Rei (UFSJ), Sao Joao del Rei; ${ }^{(d)}$ Instituto de Fisica, Universidade de Sao Paulo, Sao Paulo, Brazil 24 Physics Department, Brookhaven National Laboratory, Upton NY, United States of America

$25{ }^{(a)}$ National Institute of Physics and Nuclear Engineering, Bucharest; ${ }^{(b)}$ University Politehnica Bucharest, Bucharest; ${ }^{(c)}$ West University in Timisoara, Timisoara, Romania

${ }^{26}$ Departamento de Física, Universidad de Buenos Aires, Buenos Aires, Argentina

27 Cavendish Laboratory, University of Cambridge, Cambridge, United Kingdom

28 Department of Physics, Carleton University, Ottawa ON, Canada

29 CERN, Geneva, Switzerland

30 Enrico Fermi Institute, University of Chicago, Chicago IL, United States of America

$31{ }^{(a)}$ Departamento de Física, Pontificia Universidad Católica de Chile, Santiago;

${ }^{(b)}$ Departamento de Física, Universidad Técnica Federico Santa María, Valparaíso, Chile

32 (a) Institute of High Energy Physics, Chinese Academy of Sciences, Beijing; ${ }^{(b)}$ Department of Modern Physics, University of Science and Technology of China, Anhui; ${ }^{(c)}$ Department of Physics, Nanjing University, Jiangsu; ${ }^{(d)}$ School of Physics, Shandong University, Shandong, China

33 Laboratoire de Physique Corpusculaire, Clermont Université and Université Blaise Pascal and CNRS/IN2P3, Aubiere Cedex, France

34 Nevis Laboratory, Columbia University, Irvington NY, United States of America

35 Niels Bohr Institute, University of Copenhagen, Kobenhavn, Denmark

$36{ }^{(a)}$ INFN Gruppo Collegato di Cosenza; ${ }^{(b)}$ Dipartimento di Fisica, Università della Calabria, Arcavata di Rende, Italy

37 AGH University of Science and Technology, Faculty of Physics and Applied Computer Science, Krakow, Poland

38 The Henryk Niewodniczanski Institute of Nuclear Physics, Polish Academy of Sciences, Krakow, Poland

39 Physics Department, Southern Methodist University, Dallas TX, United States of America

40 Physics Department, University of Texas at Dallas, Richardson TX, United States of America

41 DESY, Hamburg and Zeuthen, Germany

42 Institut für Experimentelle Physik IV, Technische Universität Dortmund, Dortmund, Germany

43 Institut für Kern- und Teilchenphysik, Technical University Dresden, Dresden, Germany

44 Department of Physics, Duke University, Durham NC, United States of America

45 SUPA - School of Physics and Astronomy, University of Edinburgh, Edinburgh, United Kingdom

${ }^{46}$ Fachhochschule Wiener Neustadt, Johannes Gutenbergstrasse 32700 Wiener Neustadt, Austria

47 INFN Laboratori Nazionali di Frascati, Frascati, Italy

48 Fakultät für Mathematik und Physik, Albert-Ludwigs-Universität, Freiburg i.Br., Germany

49 Section de Physique, Université de Genève, Geneva, Switzerland

$50{ }^{(a)}$ INFN Sezione di Genova; ${ }^{(b)}$ Dipartimento di Fisica, Università di Genova, Genova, Italy

$51{ }^{(a)}$ E.Andronikashvili Institute of Physics, Tbilisi State University, Tbilisi; ${ }^{(b)}$ High Energy

Physics Institute, Tbilisi State University, Tbilisi, Georgia

52 II Physikalisches Institut, Justus-Liebig-Universität Giessen, Giessen, Germany

53 SUPA - School of Physics and Astronomy, University of Glasgow, Glasgow, United Kingdom 
54 II Physikalisches Institut, Georg-August-Universität, Göttingen, Germany

55 Laboratoire de Physique Subatomique et de Cosmologie, Université Joseph Fourier and CNRS/IN2P3 and Institut National Polytechnique de Grenoble, Grenoble, France

56 Department of Physics, Hampton University, Hampton VA, United States of America

57 Laboratory for Particle Physics and Cosmology, Harvard University, Cambridge MA, United States of America

58 (a) Kirchhoff-Institut für Physik, Ruprecht-Karls-Universität Heidelberg, Heidelberg;

${ }^{(b)}$ Physikalisches Institut, Ruprecht-Karls-Universität Heidelberg, Heidelberg; ${ }^{(c)}$ ZITI Institut für technische Informatik, Ruprecht-Karls-Universität Heidelberg, Mannheim, Germany

59 Faculty of Applied Information Science, Hiroshima Institute of Technology, Hiroshima, Japan

60 Department of Physics, Indiana University, Bloomington IN, United States of America

61 Institut für Astro- und Teilchenphysik, Leopold-Franzens-Universität, Innsbruck, Austria

62 University of Iowa, Iowa City IA, United States of America

63 Department of Physics and Astronomy, Iowa State University, Ames IA, United States of America

64 Joint Institute for Nuclear Research, JINR Dubna, Dubna, Russia

65 KEK, High Energy Accelerator Research Organization, Tsukuba, Japan

${ }^{66}$ Graduate School of Science, Kobe University, Kobe, Japan

67 Faculty of Science, Kyoto University, Kyoto, Japan

68 Kyoto University of Education, Kyoto, Japan

69 Department of Physics, Kyushu University, Fukuoka, Japan

70 Instituto de Física La Plata, Universidad Nacional de La Plata and CONICET, La Plata, Argentina

71 Physics Department, Lancaster University, Lancaster, United Kingdom

$72{ }^{(a)}$ INFN Sezione di Lecce; ${ }^{(b)}$ Dipartimento di Matematica e Fisica, Università del Salento, Lecce, Italy

73 Oliver Lodge Laboratory, University of Liverpool, Liverpool, United Kingdom

74 Department of Physics, Jožef Stefan Institute and University of Ljubljana, Ljubljana, Slovenia

75 School of Physics and Astronomy, Queen Mary University of London, London, United Kingdom

76 Department of Physics, Royal Holloway University of London, Surrey, United Kingdom

77 Department of Physics and Astronomy, University College London, London, United Kingdom

78 Laboratoire de Physique Nucléaire et de Hautes Energies, UPMC and Université Paris-Diderot and CNRS/IN2P3, Paris, France

79 Fysiska institutionen, Lunds universitet, Lund, Sweden

80 Departamento de Fisica Teorica C-15, Universidad Autonoma de Madrid, Madrid, Spain

81 Institut für Physik, Universität Mainz, Mainz, Germany

82 School of Physics and Astronomy, University of Manchester, Manchester, United Kingdom

83 CPPM, Aix-Marseille Université and CNRS/IN2P3, Marseille, France

84 Department of Physics, University of Massachusetts, Amherst MA, United States of America

85 Department of Physics, McGill University, Montreal QC, Canada

86 School of Physics, University of Melbourne, Victoria, Australia

87 Department of Physics, The University of Michigan, Ann Arbor MI, United States of America

88 Department of Physics and Astronomy, Michigan State University, East Lansing MI, United States of America

$89{ }^{(a)}$ INFN Sezione di Milano; ${ }^{(b)}$ Dipartimento di Fisica, Università di Milano, Milano, Italy

90 B.I. Stepanov Institute of Physics, National Academy of Sciences of Belarus, Minsk, Republic of Belarus 
91 National Scientific and Educational Centre for Particle and High Energy Physics, Minsk, Republic of Belarus

92 Department of Physics, Massachusetts Institute of Technology, Cambridge MA, United States of America

93 Group of Particle Physics, University of Montreal, Montreal QC, Canada

94 P.N. Lebedev Institute of Physics, Academy of Sciences, Moscow, Russia

95 Institute for Theoretical and Experimental Physics (ITEP), Moscow, Russia

96 Moscow Engineering and Physics Institute (MEPhI), Moscow, Russia

97 Skobeltsyn Institute of Nuclear Physics, Lomonosov Moscow State University, Moscow, Russia

98 Fakultät für Physik, Ludwig-Maximilians-Universität München, München, Germany

99 Max-Planck-Institut für Physik (Werner-Heisenberg-Institut), München, Germany

100 Nagasaki Institute of Applied Science, Nagasaki, Japan

101 Graduate School of Science and Kobayashi-Maskawa Institute, Nagoya University, Nagoya, Japan

$102{ }^{(a)}$ INFN Sezione di Napoli; ${ }^{(b)}$ Dipartimento di Scienze Fisiche, Università di Napoli, Napoli, Italy

103 Department of Physics and Astronomy, University of New Mexico, Albuquerque NM, United States of America

104 Institute for Mathematics, Astrophysics and Particle Physics, Radboud University Nijmegen/Nikhef, Nijmegen, Netherlands

105 Nikhef National Institute for Subatomic Physics and University of Amsterdam, Amsterdam, Netherlands

106 Department of Physics, Northern Illinois University, DeKalb IL, United States of America

107 Budker Institute of Nuclear Physics, SB RAS, Novosibirsk, Russia

108 Department of Physics, New York University, New York NY, United States of America

109 Ohio State University, Columbus OH, United States of America

110 Faculty of Science, Okayama University, Okayama, Japan

111 Homer L. Dodge Department of Physics and Astronomy, University of Oklahoma, Norman OK, United States of America

112 Department of Physics, Oklahoma State University, Stillwater OK, United States of America

113 Palacký University, RCPTM, Olomouc, Czech Republic

114 Center for High Energy Physics, University of Oregon, Eugene OR, United States of America

115 LAL, Université Paris-Sud and CNRS/IN2P3, Orsay, France

116 Graduate School of Science, Osaka University, Osaka, Japan

117 Department of Physics, University of Oslo, Oslo, Norway

118 Department of Physics, Oxford University, Oxford, United Kingdom

$119{ }^{(a)}$ INFN Sezione di Pavia; ${ }^{(b)}$ Dipartimento di Fisica, Università di Pavia, Pavia, Italy

120 Department of Physics, University of Pennsylvania, Philadelphia PA, United States of America

121 Petersburg Nuclear Physics Institute, Gatchina, Russia

$122{ }^{(a)}$ INFN Sezione di Pisa; ${ }^{(b)}$ Dipartimento di Fisica E. Fermi, Università di Pisa, Pisa, Italy

123 Department of Physics and Astronomy, University of Pittsburgh, Pittsburgh PA, United States of America

$124{ }^{(a)}$ Laboratorio de Instrumentacao e Fisica Experimental de Particulas - LIP, Lisboa, Portugal; ${ }^{(b)}$ Departamento de Fisica Teorica y del Cosmos and CAFPE, Universidad de Granada, Granada, Spain

125 Institute of Physics, Academy of Sciences of the Czech Republic, Praha, Czech Republic 
126 Faculty of Mathematics and Physics, Charles University in Prague, Praha, Czech Republic

127 Czech Technical University in Prague, Praha, Czech Republic

128 State Research Center Institute for High Energy Physics, Protvino, Russia

129 Particle Physics Department, Rutherford Appleton Laboratory, Didcot, United Kingdom

130 Physics Department, University of Regina, Regina SK, Canada

131 Ritsumeikan University, Kusatsu, Shiga, Japan

$132{ }^{(a)}$ INFN Sezione di Roma I; ${ }^{(b)}$ Dipartimento di Fisica, Università La Sapienza, Roma, Italy

133 (a) INFN Sezione di Roma Tor Vergata; ${ }^{(b)}$ Dipartimento di Fisica, Università di Roma Tor Vergata, Roma, Italy

$134{ }^{(a)}$ INFN Sezione di Roma Tre; ${ }^{(b)}$ Dipartimento di Fisica, Università Roma Tre, Roma, Italy

$135{ }^{(a)}$ Faculté des Sciences Ain Chock, Réseau Universitaire de Physique des Hautes Energies Université Hassan II, Casablanca; ${ }^{(b)}$ Centre National de l'Energie des Sciences Techniques Nucleaires, Rabat; ${ }^{(c)}$ Faculté des Sciences Semlalia, Université Cadi Ayyad, LPHEA-Marrakech; ${ }^{(d)}$ Faculté des Sciences, Université Mohamed Premier and LPTPM, Oujda; ${ }^{(e)}$ Faculté des sciences, Université Mohammed V-Agdal, Rabat, Morocco

136 DSM/IRFU (Institut de Recherches sur les Lois Fondamentales de l'Univers), CEA Saclay (Commissariat a l'Energie Atomique), Gif-sur-Yvette, France

137 Santa Cruz Institute for Particle Physics, University of California Santa Cruz, Santa Cruz CA, United States of America

138 Department of Physics, University of Washington, Seattle WA, United States of America

139 Department of Physics and Astronomy, University of Sheffield, Sheffield, United Kingdom

140 Department of Physics, Shinshu University, Nagano, Japan

141 Fachbereich Physik, Universität Siegen, Siegen, Germany

142 Department of Physics, Simon Fraser University, Burnaby BC, Canada

143 SLAC National Accelerator Laboratory, Stanford CA, United States of America

$144{ }^{(a)}$ Faculty of Mathematics, Physics \& Informatics, Comenius University, Bratislava;

${ }^{(b)}$ Department of Subnuclear Physics, Institute of Experimental Physics of the Slovak Academy of Sciences, Kosice, Slovak Republic

$145{ }^{(a)}$ Department of Physics, University of Johannesburg, Johannesburg; ${ }^{(b)}$ School of Physics, University of the Witwatersrand, Johannesburg, South Africa

$146{ }^{(a)}$ Department of Physics, Stockholm University; ${ }^{(b)}$ The Oskar Klein Centre, Stockholm, Sweden

147 Physics Department, Royal Institute of Technology, Stockholm, Sweden

148 Departments of Physics \& Astronomy and Chemistry, Stony Brook University, Stony Brook NY, United States of America

149 Department of Physics and Astronomy, University of Sussex, Brighton, United Kingdom

150 School of Physics, University of Sydney, Sydney, Australia

151 Institute of Physics, Academia Sinica, Taipei, Taiwan

152 Department of Physics, Technion: Israel Institute of Technology, Haifa, Israel

153 Raymond and Beverly Sackler School of Physics and Astronomy, Tel Aviv University, Tel Aviv, Israel

154 Department of Physics, Aristotle University of Thessaloniki, Thessaloniki, Greece

155 International Center for Elementary Particle Physics and Department of Physics, The University of Tokyo, Tokyo, Japan

156 Graduate School of Science and Technology, Tokyo Metropolitan University, Tokyo, Japan

157 Department of Physics, Tokyo Institute of Technology, Tokyo, Japan

158 Department of Physics, University of Toronto, Toronto ON, Canada 
${ }^{(a)}$ TRIUMF, Vancouver BC; ${ }^{(b)}$ Department of Physics and Astronomy, York University, Toronto ON, Canada

160 Institute of Pure and Applied Sciences, University of Tsukuba,1-1-1 Tennodai,Tsukuba, Ibaraki 305-8571, Japan

161 Science and Technology Center, Tufts University, Medford MA, United States of America

162 Centro de Investigaciones, Universidad Antonio Narino, Bogota, Colombia

163 Department of Physics and Astronomy, University of California Irvine, Irvine CA, United States of America

$164{ }^{(a)}$ INFN Gruppo Collegato di Udine; ${ }^{(b)}$ ICTP, Trieste; ${ }^{(c)}$ Dipartimento di Chimica, Fisica e Ambiente, Università di Udine, Udine, Italy

165 Department of Physics, University of Illinois, Urbana IL, United States of America

166 Department of Physics and Astronomy, University of Uppsala, Uppsala, Sweden

167 Instituto de Física Corpuscular (IFIC) and Departamento de Física Atómica, Molecular y Nuclear and Departamento de Ingeniería Electrónica and Instituto de Microelectrónica de Barcelona (IMB-CNM), University of Valencia and CSIC, Valencia, Spain

168 Department of Physics, University of British Columbia, Vancouver BC, Canada

169 Department of Physics and Astronomy, University of Victoria, Victoria BC, Canada

170 Department of Physics, University of Warwick, Coventry, United Kingdom

171 Waseda University, Tokyo, Japan

172 Department of Particle Physics, The Weizmann Institute of Science, Rehovot, Israel

173 Department of Physics, University of Wisconsin, Madison WI, United States of America

174 Fakultät für Physik und Astronomie, Julius-Maximilians-Universität, Würzburg, Germany

175 Fachbereich C Physik, Bergische Universität Wuppertal, Wuppertal, Germany

176 Department of Physics, Yale University, New Haven CT, United States of America

177 Yerevan Physics Institute, Yerevan, Armenia

178 Domaine scientifique de la Doua, Centre de Calcul CNRS/IN2P3, Villeurbanne Cedex, France

${ }^{a}$ Also at Laboratorio de Instrumentacao e Fisica Experimental de Particulas - LIP, Lisboa, Portugal

${ }^{b}$ Also at Faculdade de Ciencias and CFNUL, Universidade de Lisboa, Lisboa, Portugal

${ }^{c}$ Also at Particle Physics Department, Rutherford Appleton Laboratory, Didcot, United Kingdom

$d$ Also at TRIUMF, Vancouver BC, Canada

$e$ Also at Department of Physics, California State University, Fresno CA, United States of America

$f$ Also at Novosibirsk State University, Novosibirsk, Russia

$g$ Also at Fermilab, Batavia IL, United States of America

$h$ Also at Department of Physics, University of Coimbra, Coimbra, Portugal

$i$ Also at Department of Physics, UASLP, San Luis Potosi, Mexico

$j$ Also at Università di Napoli Parthenope, Napoli, Italy

${ }^{k}$ Also at Institute of Particle Physics (IPP), Canada

$l$ Also at Department of Physics, Middle East Technical University, Ankara, Turkey

$m$ Also at Louisiana Tech University, Ruston LA, United States of America

$n$ Also at Dep Fisica and CEFITEC of Faculdade de Ciencias e Tecnologia, Universidade Nova de Lisboa, Caparica, Portugal

o Also at Department of Physics and Astronomy, University College London, London, United Kingdom

$p$ Also at Group of Particle Physics, University of Montreal, Montreal QC, Canada

${ }^{q}$ Also at Department of Physics, University of Cape Town, Cape Town, South Africa 
${ }^{r}$ Also at Institute of Physics, Azerbaijan Academy of Sciences, Baku, Azerbaijan

$s$ Also at Institut für Experimentalphysik, Universität Hamburg, Hamburg, Germany

${ }^{t}$ Also at Manhattan College, New York NY, United States of America

$u$ Also at School of Physics, Shandong University, Shandong, China

$v$ Also at CPPM, Aix-Marseille Université and CNRS/IN2P3, Marseille, France

$w$ Also at School of Physics and Engineering, Sun Yat-sen University, Guanzhou, China

$x$ Also at Academia Sinica Grid Computing, Institute of Physics, Academia Sinica, Taipei, Taiwan

y Also at Dipartimento di Fisica, Università La Sapienza, Roma, Italy

$z$ Also at DSM/IRFU (Institut de Recherches sur les Lois Fondamentales de l'Univers), CEA Saclay (Commissariat a l'Energie Atomique), Gif-sur-Yvette, France

aa Also at Section de Physique, Université de Genève, Geneva, Switzerland

$a b$ Also at Departamento de Fisica, Universidade de Minho, Braga, Portugal

ac Also at Department of Physics and Astronomy, University of South Carolina, Columbia SC, United States of America

ad Also at Institute for Particle and Nuclear Physics, Wigner Research Centre for Physics, Budapest, Hungary

ae Also at California Institute of Technology, Pasadena CA, United States of America

af Also at Institute of Physics, Jagiellonian University, Kracow, Poland

ag Also at LAL, Université Paris-Sud and CNRS/IN2P3, Orsay, France

ah Also at Department of Physics and Astronomy, University of Sheffield, Sheffield, United Kingdom

ai Also at Department of Physics, Oxford University, Oxford, United Kingdom

aj Also at Institute of Physics, Academia Sinica, Taipei, Taiwan

ak Also at Department of Physics, The University of Michigan, Ann Arbor MI, United States of America

* Deceased 